\title{
A COMPUTATIONAL STUDY OF THE LUDWIG-SORET EFFECT ON THE THERMAL-INDUCED PHASE SEPARATION PROCESS IN POLYMER SOLUTIONS
}

\author{
by \\ Sureshkumar B. Kukadiya \\ Bachelor of Chemical Engineering, Gujarat University, India, 2000
}

\author{
A thesis \\ presented to Ryerson University \\ in partial fulfillment of the \\ requirement for the degree of \\ Master in Applied Science \\ in the program of \\ Chemical Engineering
}

Toronto, Ontario, Canada, 2008

(C)Sureshkumar B. Kukadiya, 2008

MrYmT OF

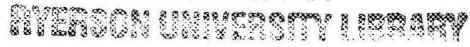




\section{AUTHOR'S DECLARATION}

I hereby declare that I am the sole author of this thesis.

I authorize the Ryerson University to lend this thesis to other institutions or individuals for the purpose of scholarly research.

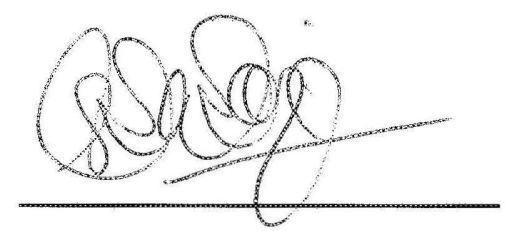

Sureshkumar B. Kukadiya

I further authorize the Ryerson University to reproduce this thesis by photocopying or by any other means, in total or in part, at the request of other institutions or individuals for the purpose of scholarly research.

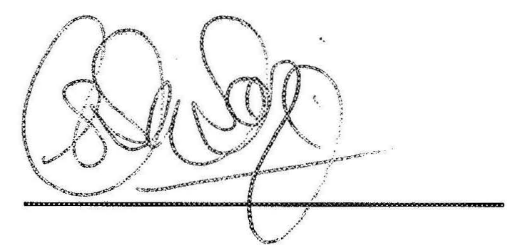

Sureshkumar B. Kukadiya 


\title{
ABSTRACT
}

\section{A COMPUTATIONAL STUDY OF THE LUDWIG-SORET EFFECT ON THE THERMAL-INDUCED PHASE SEPARATION PROCESS IN POLYMER SOLUTIONS}

\author{
Sureshkumar B. Kukadiya, MASc, 2008. \\ Department of Chemical Engineering, Ryerson University. \\ 350 Victoria Street, Toronto, Ontario M5B 2K3 Canada.
}

Thermal-induced phase separation (TIPS) is one of the methods used to fabricate functional polymeric materials, i.e. PDLC films for electro-optical devices such flat-panel displays, switchable windows etc., and microporous synthetic membranes from polymer solutions. Since the characteristic thermal, mechanical, and optical properties of these materials are controlled by the morphological features, it is important to understand the phase separation mechanism that forms these materials. In this work, the effect of thermal diffusion, also known as the Ludwig-Soret effect, on the TIPS method of phase separation via the SD mechanism in polymer solutions under non-uniform temperature field has been investigated using the computational technique. The Ludwig-Soret effect occurs when a temperature gradient applied to a fluid mixture induces a net mass flow, which leads to the formation of a concentration gradient. A rigorous mathematical model for TIPS via

the spinodal decomposition mechanism based on the nonlinear Cahn-Hilliard and FloryHuggins theories combined with thermal diffusion phenomenon has been formulated for binary polymer solutions under non-uniform temperature field and solved numerically. Numerical simulation results revealed that the thermal diffusion phenomenon had very little or negligible effect on the phase separation mechanism under a non-uniform temperature field, which was reflected from the studies of the time evolution of structure factor and transition time from the early to the intermediate stages of SD. 


\section{ACKNOWLEDGEMENTS}

I would like to express my deepest gratitude to my supervisor, Dr. Philip K. Chan and my co-supervisor, Dr. Mehrab Mehrvar for giving me an opportunity to work on this research thesis. My special thanks go to Dr. Philip Chan for his thoughtful guidance, patience, encouragement and continual support throughout this research work.

I am also very thankful to all my friends, at the Ryerson University and especially to all my group members, Anthony Jiang, Danny Lee, and Mohammad T. of Dr. Philip Chan's research group, who assisted me in the research endeavor by providing conducive environment through active theoretical discussions. I am also very thankful to the support staff of the department of chemical engineering at Ryerson University.

I would also like to acknowledge the financial supports from the Natural Sciences \& Engineering Research Council (NSERC) of Canada, Ontario Graduate Scholarship for Science \& Technology (OGSST) and Ryerson University for this research thesis.

Finally, I am very grateful to my wife, Sima for her constant support and encouragement during my tenure at Ryerson University. Without her love, support and motivation, the completion of this thesis would not be possible. 


\section{DEDICATION}

To

MY BELOVED WIFE

who has always been

a constant source of encouragement and support. 


\section{TABLE OF CONTENTS}

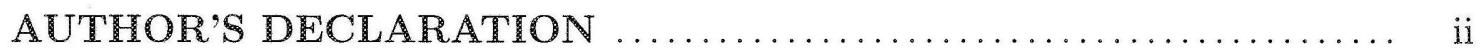

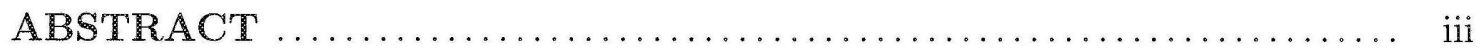

ACKNOWLEDGEMENTS $\ldots \ldots \ldots \ldots \ldots \ldots \ldots \ldots \ldots \ldots \ldots \ldots \ldots \ldots \ldots \ldots \ldots$

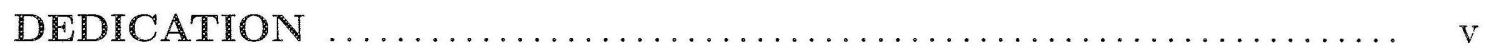

LIST OF FIGUPES $\ldots \ldots \ldots \ldots \ldots \ldots \ldots \ldots \ldots \ldots \ldots \ldots \ldots \ldots \ldots \ldots \ldots \ldots$ viii

LIST OF TABLES $\ldots \ldots \ldots \ldots \ldots \ldots \ldots \ldots \ldots \ldots \ldots \ldots \ldots \ldots \ldots \ldots \ldots \ldots \ldots \ldots \ldots \ldots$

NOMENCLATURE $\ldots \ldots \ldots \ldots \ldots \ldots \ldots \ldots \ldots \ldots \ldots \ldots \ldots \ldots \ldots \ldots \ldots \ldots \ldots$

CHAPTER $1 \quad$ GENERAL INTRODUCTION $\ldots \ldots \ldots \ldots \ldots \ldots \ldots \ldots$

1.1 Phase Separation in Polymer Solutions . . . . . . . . . . . . . . . . . 1

1.2 Phase Separation Mechanisms ............................ 3

1.3 Methods of Phase Separation $\ldots \ldots \ldots \ldots \ldots \ldots \ldots \ldots \ldots \ldots \ldots \ldots$

1.3.1 Thermal-Induced Phase Separation (TIPS) Method .......... 9

1.3.2 Polymerization-Induced Phase Separation (PIPS) method ...... 11

1.4 The Phenomenon of Ludwig-Soret Effect . . . . . . . . . . . . . 13

CHAPTER $2 \quad$ LTERATURE REVIEW ................... 15

2.1 Thermal-Induced Phase Separation Method ................... 15

2.1.1 Polymer Dispersed Liquid Crystal (PDLC) Films . . . . . . . . . 17

2.1.2 Microporous Synthetic Membranes ...................... 19

2.2 Thermal Diffusion Phenomenon in Polymers.................. 25

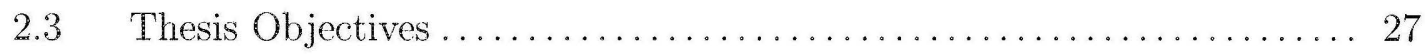

CHAPTER $3 \quad$ MODEL DEVELOPMENT ...................... 29

3.1 Thermodynamics in Polymer Solutions . . . . . . . . . . . . . . . . . 29

3.1.1 The Flory-Huggins Theory . . . . . . . . . . . . . . 30

3.1.2 Phase Equilibria and Phase Diagram ................ 32 


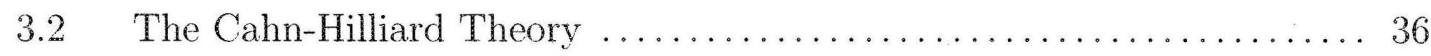

3.3 Mutual and Self-Diffusion Coefficients ...................... 38

3.4 Concentration Dependent Mobility ......................... 41

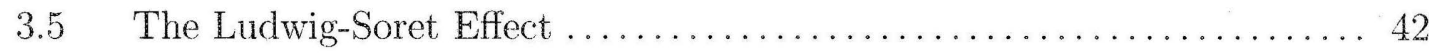

\section{CHAPTER 4 GOVERNING EQUATIONS AND METHOD OF} SOLUTIONS ....................................... 47

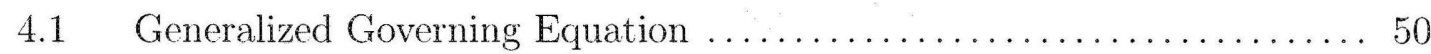

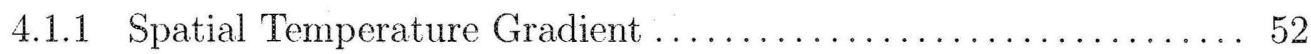

4.1.2 Concentration Dependent Mobility ..................... 53

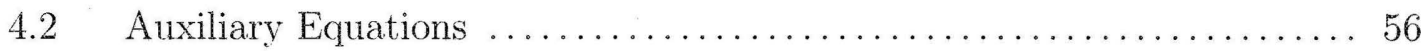

4.2 .1 Boundary Conditions............................. 57

4.3 Method of Solution ................................. 58

4.3.1 Galerkin Finite Element Method ........................ 59

4.3.2 Euler's Predictor-Corrector Method .................... 61

4.3 .3 Time Step-Size Control ........................... 63

CHAPTER 5 RESULTS AND DISCUSSION ................. 66

$5.1 \quad$ The Structures and Patterns Formation $\ldots \ldots \ldots \ldots \ldots \ldots \ldots \ldots 6$

5.2 Time Evolution of Dimensionless Structure Factor ............... 75

5.3 The Transition Time from the Early Stage to the Intermediate Stage .. 82

CHAPTER 6 CONCLUSIONS .............................. 85

CHAPTER 7 RECOMMENDATIONS $\ldots \ldots \ldots \ldots \ldots$

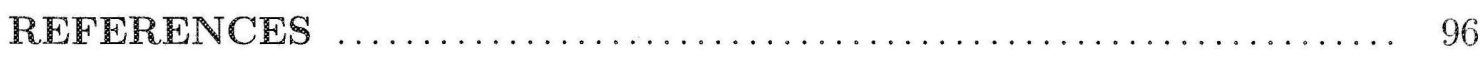

CHAPTER A DIMENSIONLESS GOVERNING EQUATIONS ..... 97

CHAPTER B ESTIMATION OF ORDER OF MAGNITUDE...... 99 


\section{LIST OF FIGURES}

1.1 Schematic of a typical phase diagram of a binary polymer solution showing the binodal curve and the spinodal curve $[1] \ldots \ldots \ldots \ldots \ldots \ldots \ldots \ldots \ldots$

1.2 A typical phase diagram (temperature, $T$ versus solvent concentration, $c$ ) of a binary polymer solution showing different morphologies that can be obtained depending on the location of the quench point $(c, T)$ on the phase diagram [1]

1.3 A schematic of one-dimensional spatial concentration profile of the phase separation by the Nucleation and Growth (NG) mechanism from the initial concentration fluctuations at time, $t_{1}$ to the development and growth of domain sizes at time, $t_{3} . c_{U}$ and $c_{L}$ are the high and low equilibrium concentrations on the binodal curve, respectively and $c_{o}$ is the initial average concentration of the polymer solution $[2,3]$. $\left(t_{1}<t_{2}<t_{3}\right) \ldots \ldots \ldots \ldots \ldots$.

1.4 A schematic of one-dimensional spatial concentration profile of the phase separation by the Spinodal Decomposition (SD) mechanism from the initial concentration fluctuations at time, $t_{1}$ to the development and growth of domain sizes at time, $t_{3} . c_{U}$ and $c_{L}$ are the high and low equilibrium concentrations on the binodal curve, respectively and $c_{o}$ is the initial average concentration of the polymer solution $[1,2,3]$. $\left(t_{1}<t_{2}<t_{3}\right) \ldots \ldots \ldots \ldots \ldots$

1.5 A schematic of the phase diagram showing the phase separation by TIPS method via the SD mechanism, which involves the quenching of a homogeneous single phase polymer solution at an average initial concentration, $c_{0}$ (open circles) from its initial temperature, $T_{i}$ to the final temperature, $T_{f}$. In the case of the critical quench (route $B$ ), the resultant morphology is of interconnected-type structures, and in the case of the off-critical quench (route $\mathrm{A}$ or $\mathrm{C}$ ), the droplet type morphology results. $c_{c}$ represents the critical concentration of the polymer solution $[2] \ldots \ldots \ldots \ldots \ldots \ldots \ldots 1$ 
1.6 A typical phase diagram showing characteristic properties of the PIPS method. As the solute (polymer) degree of polymerization, $N_{2}$ increases, the phase diagram shifts upward (in the direction of the arrow). The phase separation begins as soon as the cure point (black dot) enters the unstable region or spinodal region of the phase diagram $[4,2]$. $\left(t_{1}<t_{2}<t_{3}\right) \ldots \ldots \ldots \ldots \ldots \ldots$

3.1 A Schematic diagram of the Gibbs free energy of mixing as a function of the solvent concentration (top half) and typical schematic symmetrical phase diagram for a binary solution (bottom half) showing the metastable region (dotted area) and unstable spinodal region (gray shaded area). The contact points for the common tangents drawn to the minima are shown projected onto the temperature-concentration plane to form the binodal (cloud point) curve, whereas projection of the inflexion points forms the spinodal curve. The upper diagram shows the different Gibbs free energy curves corresponding to the temperatures $T_{1}$ to $T_{4}\left(T_{1}>T_{2}>T_{3}>T_{4}\right)[5] \ldots \ldots \ldots \ldots \ldots \ldots$

4.1 A flowchart of mathematical modelling and computational simulation procedures for the accomplishment of the objectives of the research thesis..... 48

4.2 The first order implicit Euler's Predictor-Corrector method [6] ........ 62

5.1 An asymmetric phase diagram for a binary polymer solution calculated from the Flory-Huggins theory using $N_{1}=1, \& N_{2}=10$. The solid curve is the binodal curve, and the dashed curve is the spinodal curve. The $c^{*}$ coordinates of the initial conditions used in the simulations are: 0.6 for interval AB (off-critical quench), 0.7597 for interval CD (critical quench) and 0.8 for interval EF (off-critical quench). Intervals $\mathrm{AB}, \mathrm{CD}$, and EF represent the overall depth of the spatial linear temperature gradient imposed on the

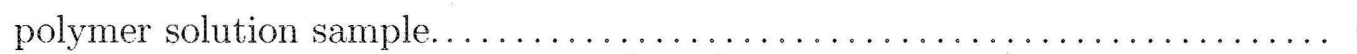

5.2 Spatial concentration profiles, $c^{*}\left(x^{*}, y^{*}\right)$ (first column) and patterns (second column) corresponding to the interval $\mathrm{AB}$ (refer to Figure 5.1) for $D^{*}=8 \times$ $10^{3}$ and $D^{\prime *}=1.0 \times 10^{5}$ at the following dimensionless times, $t^{*}: 5.38 \times 10^{-5}$ (first row), $6.87 \times 10^{-5}$ (second row) and $8.01 \times 10^{-5}$ (third row). The black regions (white regions) in patterns represent solute-rich regions (solvent-rich regions) with $c^{*}<c_{c}^{*}\left(c^{*}>c_{c}^{*}\right)$, where $c_{c}^{*}=0.7597$ denotes the dimensionless critical concentration. The figure corresponds to a non-uniform off-critical quench case with $c_{0}^{*}=0.60$, and the resultant pattern is of droplet-type.... 
5.3 Spatial concentration profiles, $c^{*}\left(x^{*}, y^{*}\right)$ (first column) and patterns (second column) corresponding to the interval CD (refer to Figure 5.1) for $D^{*}=$ $8 \times 10^{3}$ and $D^{\prime *}=1.0 \times 10^{5}$ at the following dimensionless times, $t^{*}: 2.98 \times$ $10^{-5}$ (first row), $3.44 \times 10^{-5}$ (second row) and $6.93 \times 10^{-5}$ (third row). The black regions (white regions) in patterns represent solute-rich regions (solvent-rich regions) with $c^{*}<c_{c}^{*}\left(c^{*}>c_{c}^{*}\right)$, where $c_{c}^{*}=0.7597$ denotes the dimensionless critical concentration. The figure corresponds to a nonuniform critical quench case with $c_{0}^{*}=0.7597$, and the resultant pattern is of transient interconnected-type structures....................... 7

5.4 Spatial concentration profiles, $c^{*}\left(x^{*}, y^{*}\right)$ (first column) and patterns (second column) corresponding to the interval EF (refer to Figure 5.1) for $D^{*}=$ $8 \times 10^{3}$ and $D^{\prime *}=1.0 \times 10^{5}$ at the following dimensionless times, $t^{*}: 6.68 \times$ $10^{-5}$ (first row), $7.86 \times 10^{-5}$ (second row) and $10.58 \times 10^{-5}$ (third row). The black regions (white regions) in patterns represent solute-rich regions (solvent-rich regions) with $c^{*}<c_{c}^{*}\left(c^{*}>c_{c}^{*}\right)$, where $c_{c}^{*}=0.7597$ denotes the dimensionless critical concentration. The figure corresponds to a nonuniform critical quench case with $c_{0}^{*}=0.80$, and the resultant pattern is of droplet-type. .........................................

5.5 Typical light scattering profiles showing the time evolution of phase separation by the SD mechanism in the early stage to the beginning of the intermediate stage in the small angle light scattering (SALS) experiment. Each profile represents scattered light intensity at certain time, $t$. The scattered light intensity, $I_{s}$ is increasing with time, while the wave number, $q$ remains constant, which is typical characteristic of the early stage of SD [7].. 77

5.6 The time evolution of the dimensionless structure factor, $S^{*}\left(\mathbf{k}^{*}, t^{*}\right)$ for the simulation corresponding to the thermal diffusion coefficient, $D^{\prime *}=0$ (refer to case 1 in Table 5.2 for other parameter values). .................. 79

5.7 The time evolution of the dimensionless structure factor, $S^{*}\left(\mathbf{k}^{*}, t^{*}\right)$ for the simulation corresponding to the thermal diffusion coefficient, $D^{\prime *}=1.0 \times 10^{5}$ (refer to case 2 in Table 5.2 for other parameter values) .............. 80

5.8. The time evolution of the dimensionless structure factor, $S^{*}\left(\mathbf{k}^{*}, t^{*}\right)$ for the simulation corresponding to the thermal diffusion coefficient, $D^{\prime *}=3.0 \times 10^{5}$ (refer to case 3 in Table 5.2 for other parameter values). 
5.9 The time evolution of the dimensionless structure factor, $S^{*}\left(\mathbf{k}^{*}, t^{*}\right)$ for the simulation corresponding to the thermal diffusion coefficient, $D^{\prime *}=5.0 \times 10^{5}$

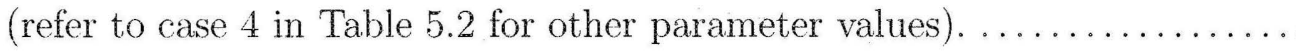

5.10 A semilog plot of the time evolution of the dimensionless maximum structure factor, $S^{*}\left(\mathbf{k}_{m}^{*}, t^{*}\right)$ versus the dimensionless time, $t^{*}$ for a non-uniform quench case of polymer phase separation mechanism (TIPS via SD) in polymer solutions. This curve is typical of the spinodal decomposition (SD) mechanism, since there is an exponential growth initially at early stage, and then it slows down when it enters the intermediate stage of the phase separation mechanism. 


\section{LIST OF TABLES}

5.1 Parameter values of the selected initial conditions (see Figure 5.1) .....68

5.2 Dimensionless material parameters used in the simulation study of the investigation of the effect of thermal diffusion phenomenon on TIPS via the SD mechanism under an externally imposed spatial linear temperature gradient in binary polymer solutions. . . . . . . . . . . . . . . $78 \ldots \ldots \ldots \ldots$

5.3 Transition time, $t_{t}^{*}$ corresponding to the simulation cases listed in Table 5.2. 84

B.1 Parameter values 100 


\section{NOMENCLATURE}

\section{Symbols}

$A(\mathbf{k}, t)$

c

$c_{C}$

$c_{i}$

$c_{0}$

CU

$c_{L}$

$c^{\alpha}$

$c^{\beta}$

D

$D^{\prime}$

$D^{\prime \prime}$

$D_{1}$

$D_{2}$

$f(c)$

F

$F_{i}$

$G_{1}$

$G_{2}$

$G_{12}$

J

$J_{q}^{\prime}$

\section{Description}

Magnitude of the Fourier Transform of concentration fluctuations

Solvent concentration (volume fraction)

Critical solvent concentration (volume fraction)

Concentration of $i^{\text {th }}$ component in the solution (volume fraction)

Initial average solvent concentration (volume fraction)

Upper equilibrium value of solvent concentration (volume fraction)

Lower equilibrium value of solvent concentration (volume fraction)

solvent concentration in $\alpha$ phase of polymer solution (volume fraction)

solvent concentration in $\beta$ phase of polymer solution (volume fraction)

Mutual diffusion coefficient $\left(\mathrm{m}^{2} \mathrm{~s}^{-1}\right)$

Thermal diffusion coefficient $\left(\mathrm{m}^{2} \mathrm{~s}^{-1} \mathrm{~K}^{-1}\right)$

Dufour coefficient $\left(\mathrm{m}^{2} \mathrm{~s}^{-1} \mathrm{~K}^{-1}\right)$

Self-diffusion coefficient of solvent $\left(\mathrm{m}^{2} \mathrm{~s}^{-1}\right)$

Self-diffusion coefficient of solute (polymer) $\left(\mathrm{m}^{2} \mathrm{~s}^{-1}\right)$

Flory-Huggins free energy of mixing ( $\mathrm{J}$ or $\mathrm{kg} \mathrm{m}^{2} \mathrm{~s}^{-2}$ )

Total free energy of mixing ( $\mathrm{J}$ or $\mathrm{kg} \mathrm{m}^{2} \mathrm{~s}^{-2}$ )

Weighted residual vector, $i=1,2,3, \ldots N$

Gibbs free energy of component $1\left(\mathrm{~J}\right.$ or $\left.\mathrm{kg} \mathrm{m}^{2} \mathrm{~s}^{-2}\right)$

Gibbs free energy of component 2 ( $\mathrm{J}$ or $\mathrm{kg} \mathrm{m}^{2} \mathrm{~s}^{-2}$ )

Gibbs free energy of solution ( $\mathrm{J}$ or $\mathrm{kg} \mathrm{m}^{2} \mathrm{~s}^{-2}$ )

Interdiffusional flux $\left(\mathrm{mol} \mathrm{m}^{-2} \mathrm{~s}^{-1}\right)$

Coefficient related to heat flow $\left(\mathrm{J} \mathrm{m}^{-2} \mathrm{~s}^{-1}\right)$ 


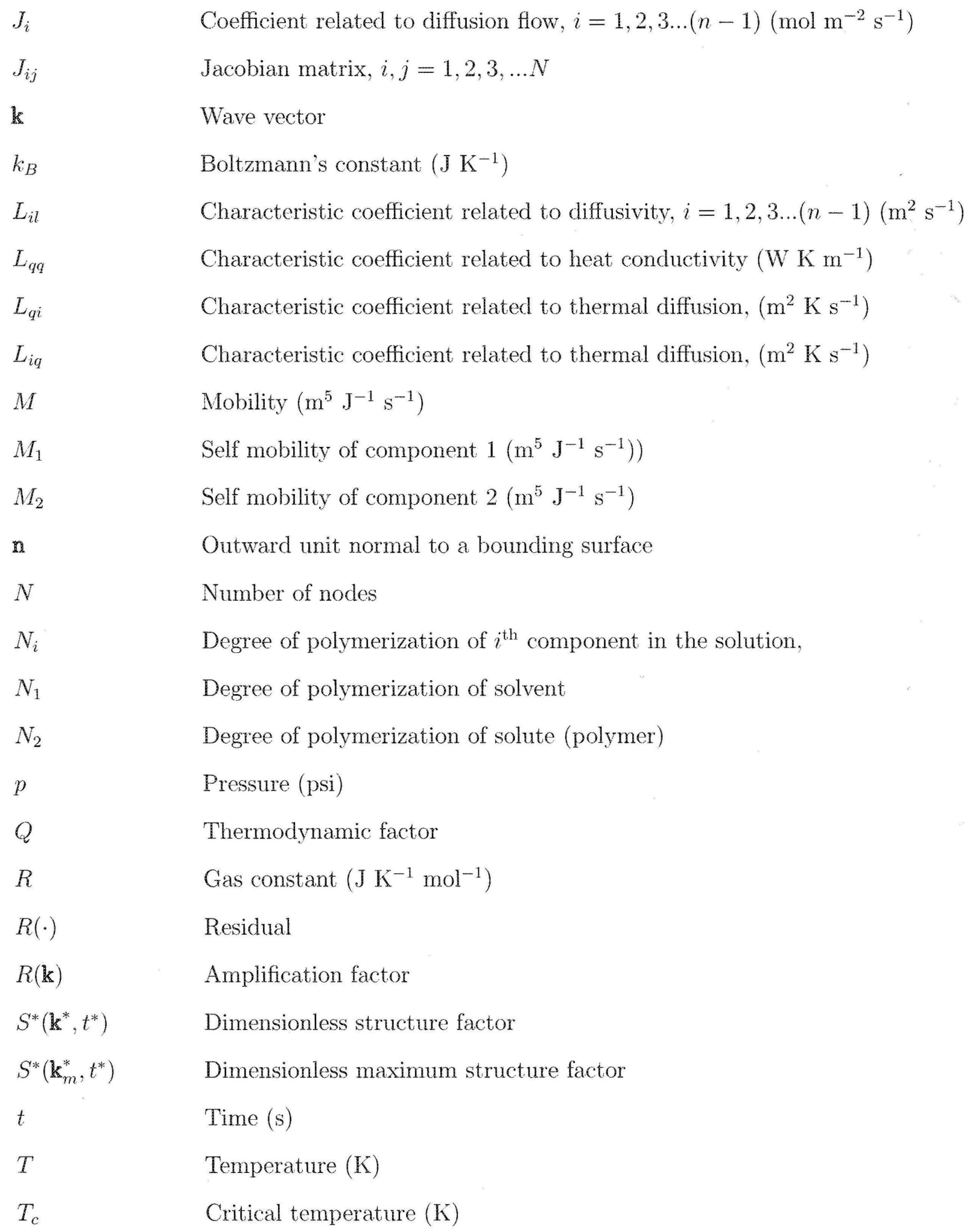


Final temperature $(\mathrm{K})$

$T_{i}$

Initial temperature $(\mathrm{K})$

$u_{a}$

Approximate solution

$u_{j}$

Set of unknown coefficients, $j=1,2,3, \ldots N$

V

Total volume of polymer solution $\left(\mathrm{m}^{3}\right)$

$w_{j}$

Set of weighting function, $j=1,2,3, \ldots N$

$x, y$

Distance of rectangular co-ordinates $(\mathrm{m})$

$\Delta G^{M}$

Change in Gibbs free energy of mixing $(\mathrm{J})$

$\Delta H^{M}$

Change in enthalpy of mixing $(J)$

$\Delta S^{M}$

Change in entropy of mixing $\left(\mathrm{J} \mathrm{K}^{-1}\right)$

\section{Greek Letters Description}
$\alpha$
A scalar
$\vec{\beta}$
A vector
$\chi$
Flory's interaction Parameter (dimensionless)
$\chi_{c}$
Critical value of Flory's interaction parameter (dimensionless)
$\Delta \mu_{1}^{*}$
Dimensionless change of the solvent chemical potential
$\kappa$
Gradient energy coefficient $\left(\mathrm{J} \mathrm{m}^{-1}\right)$
$\lambda$
Wavelength for fluctuation (angstrom)
$\Lambda$
Kinetic factor (Onsager coefficient)
Dimensionless standard chemical potential of pure solvent
$\mu_{1}$
Chemical potential of solvent $\left(\mathrm{J} \mathrm{mol}^{-1}\right)$
$\mu_{2}$
Chemical potential of polymer solute $\left(\mathrm{J} \mathrm{mol}^{-1}\right)$
Volume of a cell or segment $\left(\mathrm{m}^{3}\right)$
$\Omega$
Number of possible arrangements in the lattice space
$\phi_{j}$
Set of linearly independent functions, $j=1,2,3, \ldots N$ 
$\Phi_{j}$

$\phi_{1}$

$\phi_{2}$

$\psi$

$\sigma$

$\theta$

$\xi_{i}$

$\xi_{1}$

$\xi_{2}$

\section{Superscripts Description}

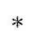

$\dagger, \dagger$
Set of Hermitian bicubic basis function, $j=1,2,3, \ldots N$

Volume fractions of component 1

Volume fractions of component 2

Dimensionless entropy of dilution parameter (dimensionless)

Entropy production $\left(\mathrm{J} \mathrm{K}^{-1} \mathrm{~S}^{-1}\right)$

Theta temperature $(\mathrm{K})$

Frictional coefficient of $i^{\text {th }}$ component per segment in the solution $\left(\mathrm{J} \mathrm{s} \mathrm{m}^{-2}\right)$

Frictional coefficient of a component 1 per segment $\left(\mathrm{J} \mathrm{s} \mathrm{m}^{-2}\right)$

Frictional coefficient of a component 2 per segment $\left(\mathrm{J} \mathrm{s} \mathrm{m}^{-2}\right)$

Dimensionless Variable

Respective phases of polymer solution 


\section{CHAPTER 1}

\section{GENERAL INTRODUCTION}

Phase separation in polymer solutions or polymer blends has attracted a great amount of interest among scientists in recent years. Phase separation in polymer solution is one of the most important and very well known phenomena used for manufacturing functional polymeric materials, which are extensively used in engineering applications. Some of the typical examples include micro-porous synthetic (polymeric) membranes and polymer dispersed liquid crystals (PDLC) films for electro-optical devices, such as flat-panel displays for computer monitors, televisions; switchable or privacy windows etc. Due to the widespread demand and scope of the unique thermal, mechanical and optical properties of these materials, there has been continuous research and development for the modification of these materials for better properties and functionalities for the last two decades. Therefore, it is very important to understand the nonlinear phenomena that form these special materials. This chapter describes the methods used to fabricate these materials. It also describes the phenomenon of the 'Ludwig-Soret effect' or 'Thermal Diffusion' as it contributes to the process of phase separation along with the non-Fickian diffusion (uphill diffusion), under the influence of an externally imposed spatial linear temperature gradient.

\subsection{Phase Separation in Polymer Solutions}

Phase separation processes in polymer solutions or polymer blends are an important and well-studied problems in many areas of materials science. Phase separation in polymer 
solutions, often called as a process of 'de-mixing', involves the transformation of the homogeneous polymer solution in its single-phase state to the inhomogeneous two-phase state, i.e. two-phase separated regions. Such a kind of phase separation can be achieved either by the Nucleation and Growth (NG) mechanism or by the Spinodal Decomposition (SD) mechanism $[1,8]$, which will be discussed in detail in the next section of this chapter. Specifically, for binary polymer solutions, two-phase separated regions consist of the polymer-rich and solvent-rich regions. It is very important to stabilize the phase separated structure of a polymer solution after the process of phase separation in order to utilize its morphological properties to the greatest effect. This is carried out by the process of crystallization, vitrification or gelation of the polymer-rich phase depending on the endapplication of the polymer solution. After the structural stabilization, material transforms into a porous membrane if the solvent is removed from the material either by solvent evaporation, extraction, or freeze drying. It can also be transformed into a PDLC film if a low molecular weight liquid crystal (LC) is used as a solvent. There are two very popular methods used for the initiation of the phase separation process for the formation of anisotropic polymeric materials, namely the Thermal Induced Phase Separation (TIPS) method and the Polymerization Induced Phase Separation (PIPS) method $[9,10,11]$.

The structural anisotropy can be introduced by imposing an external fluctuation, such as an electric field $[12,13]$, a shear flow $[14,15]$, a concentration gradient $[16,17,18,19]$, a temperature gradient $[20,21,22]$, or a controlled chemical reaction $[23,24,25,26]$. In the TIPS method, the phase separation process is initiated or induced by rapidly changing the temperature of the solution, or by introducing the temperature gradient across a polymer solution and in the PIPS method, as the name suggests, the phase separation is induced by converting the reactive monomer molecules into polymer through the polymerization process. 


\subsection{Phase Separation Mechanisms}

Phase separation mechanism is usually driven by the thermodynamics of the polymer solution, but kinetic processes like nucleation and molecular diffusion often determine the nature of the process. As mentioned in the previous section, two distinct mechanisms are recognized in the phase separation process, the Nucleation and Growth (NG) mechanism and the Spinodal Decomposition (SD) mechanism [5]. Figure 1.1 shows a schematic of a typical phase diagram of a binary polymer solution, which is a plot of temperature, $T$ versus solvent concentration, $c$. The solid curve represents the binodal curve, often called

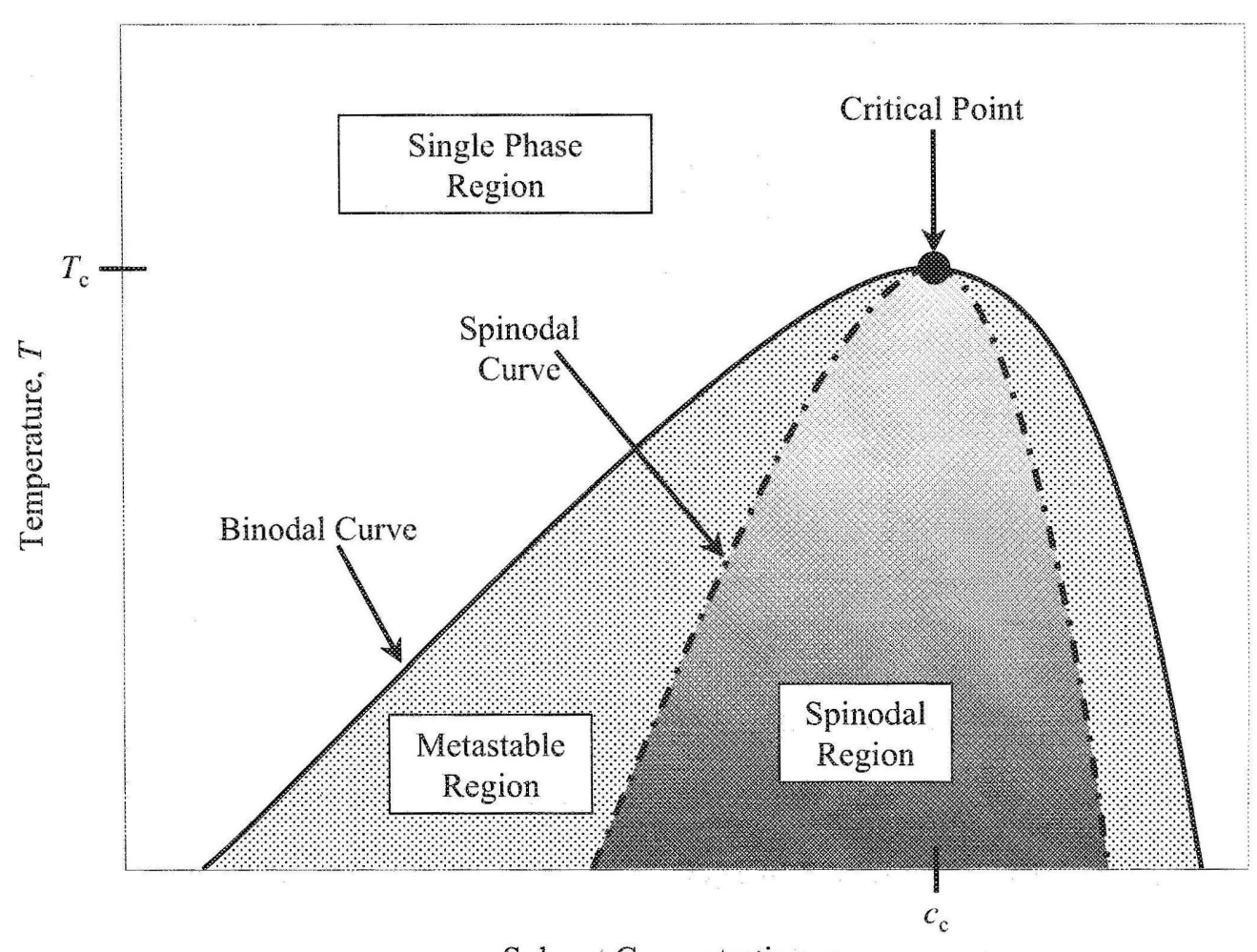

Solvent Concentration, $c$

Figure 1.1: Schematic of a typical phase diagram of a binary polymer solution showing the binodal curve and the spinodal curve [1]. 
as an equilibrium curve and the dash-dot curve represents the spinodal curve. The phase diagram is asymmetrical and it shows the upper critical solution temperature (UCST) behavior of a polymer solution, which is typical of a binary polymer solution. The area under the spinodal curve (shaded area in gray as shown in Figure 1.1) is called the spinodal or unstable region and the area under the binodal curve and outside the spinodal curve is called the metastable region (shaded area with fine dots as shown in Figure 1.1). The point, where the binodal and spinodal curves meet each other, is called the critical point (a dark filled dot as shown in Figure 1.1) and the corresponding concentration and temperature at the point are called the critical concentration, $c_{c}$, and the critical temperature, $T_{c}$, respectively. The area, outside the binodal curve, represents the region of homogeneous single phase.

If the system requires an activation energy for the initiation of the new phase, then the phase separation proceeds by the Nucleation and Growth (NG) mechanism [27]. This process usually occurs beneath the binodal curve in the metastable region of the phase diagram as shown in Figure 1.1. Alternatively, under the spinodal curve, the phase separation occurs without an activation barrier. This lack of activation barrier changes the nature of the process significantly, leading to the Spinodal Decomposition (SD) mechanism [27]. Which phase separation route the system chooses depends on the size of the gap separating the binodal curve and the spinodal curve, the size of the activation barrier within the metastable region, whether nucleation sites are available, and the rapidity with which the system crosses the phase boundary line. Such factors are often critical in PDLC systems or microporous synthetic polymeric membranes, as the nucleation and growth $(\mathrm{NG})$ and the spinodal decomposition (SD) mechanisms can lead to different types of polymer morphologies in the final film as shown in Figure 1.2.

Figure 1.2 is a typical phase diagram (temperature, $T$ versus solvent concentration, $c$ ) of 


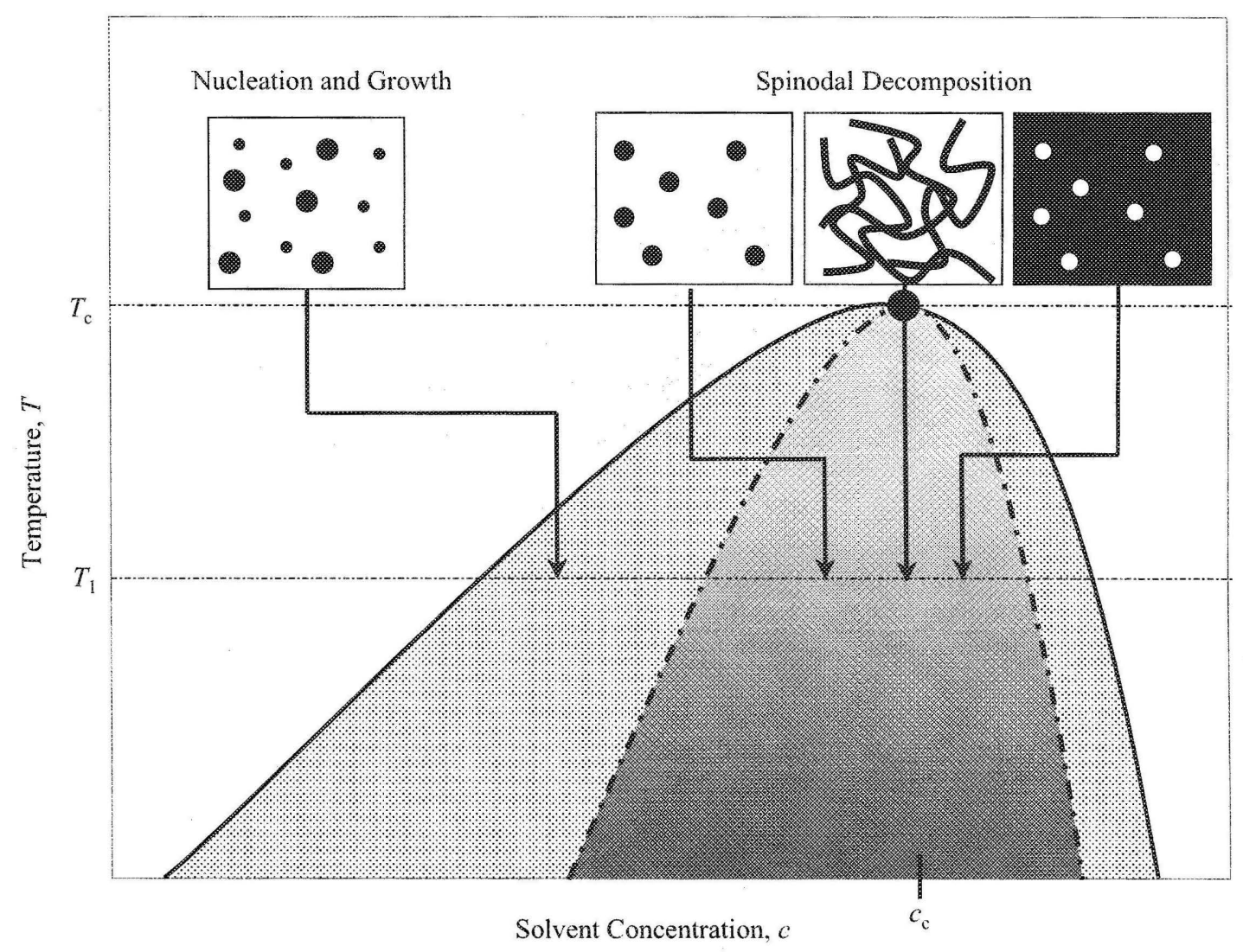

Figure 1.2: A typical phase diagram (temperature, $T$ versus solvent concentration, $c$ ) of a binary polymer solution showing different morphologies that can be obtained depending on the location of the quench point $(c, T)$ on the phase diagram [1].

a binary polymer solution showing different morphologies that can be obtained depending on the location of the quench point $(c, T)$ on the phase diagram. If the quench point $(c, T)$ of the polymer solution lies in the metastable region of the phase diagram, the phase separation occurs by the nucleation and growth $(\mathrm{NG})$ mechanism, which produces randomly distributed droplet-type morphology that grows over time due to the increase in free energy of the solution. If the quench point $(c, T)$ of the polymer solution lies in the spinodal region or unstable region of the phase diagram, the phase separation occurs by the SD mechanism due to existing infinitesimal concentration fluctuations that drive or initiate the phase separation in the SD region and two kinds of morphologies are formed: the 
droplet-type (off critical quench) and the interconnected-type (critical quench) as shown in Figure 1.2. Depending on the location of the quench point $(c, T)$, the droplet type structure has a continuous phase and dispersed phase as indicated by the reversal of black and white regions as shown in the SD structures in Figure 1.2.

Figures 1.3 and 1.4 show the one-dimensional spatial concentration profile of phase separation by NG and SD, respectively. In the metastable region of the phase diagram, an increase in the free energy of the solution due to the sufficient increase in the concentration fluctuations in the polymer solution causes the phase separation to occur by the $\mathrm{NG}$ mechanism. Consequently, phase separated droplet-type morphology develops and grows over time, where the direction of the diffusional flux is said to be downhill (from higher concentration to lower concentration) as indicated by the downward arrows in Figure 1.3 at time, $t_{1}$ (downhill diffusion - typical of NG). Pattern formation as a result of the phase separation by the NG mechanism is shown in Figure 1.3 during the times, $t_{2}$ and $t_{3}$, which is indicative of the development and growth of the phase separated regions or domains over the time. In the spinodal region or unstable region of the phase diagram, infinitesimal concentration fluctuations in the polymer solution are sufficient to drive or initiate the phase separation by the SD mechanism. Thus, phase separation by the SD mechanism is a spontaneous process as there is no need of activation energy to overcome any energy barrier. The direction of the diffusional flux is said to be uphill (from low concentration to higher concentration) as indicated by the upward arrows in Figure 1.4 at time, $t_{1}$ (uphill diffusion - typical of SD). The phase separation process by the SD mechanism is comprised of three stages: early stage, intermediate stage and late stage (refer to Figure 1.4). The phase separated regions (droplet/interconnected morphology) grow over the time from initial stage to intermediate stage and become larger and larger as time passes due to coarsening of the phase separated regions at the late stage of phase separation by SD. 


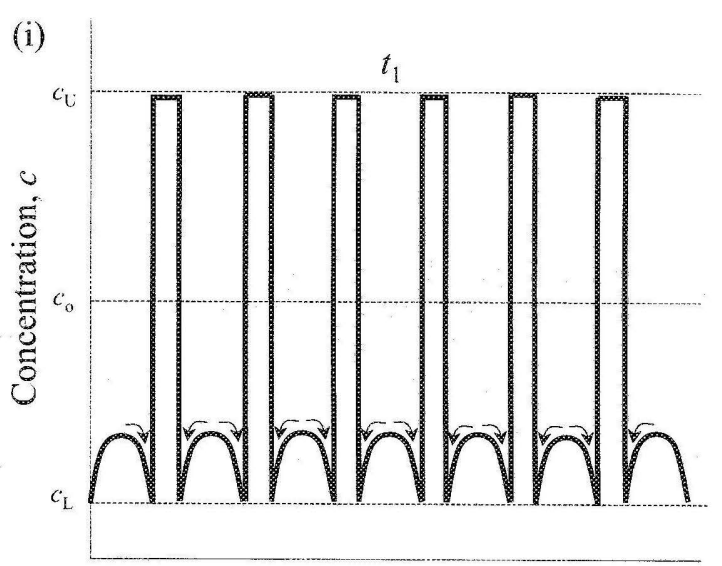

Distance, $x$

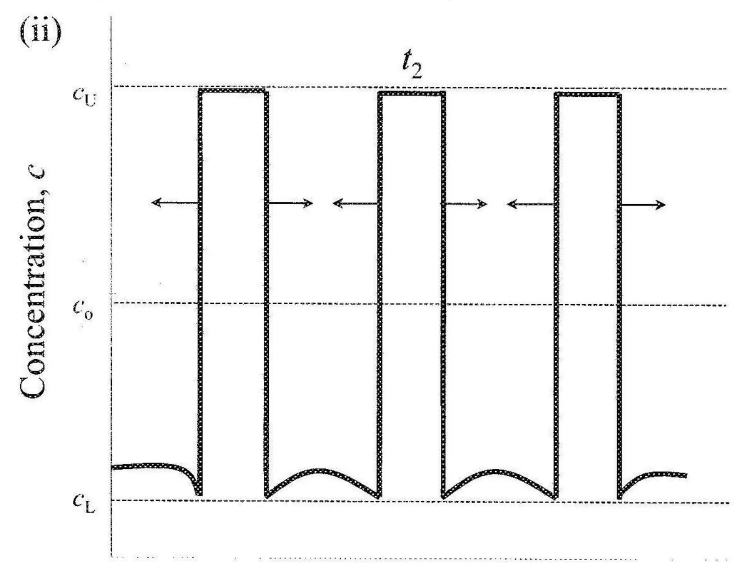

Distance, $x$

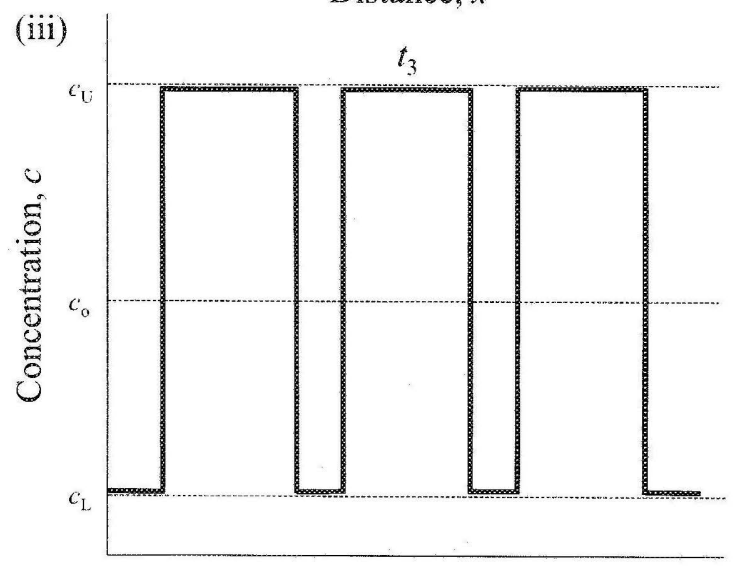

Distance, $x$

Figure 1.3: A schematic of one-dimensional spatial concentration profile of the phase separation by the Nucleation and Growth (NG) mechanism from the initial concentration fluctuations at time, $t_{1}$ to the development and growth of domain sizes at time, $t_{3} . c_{U}$ and $c_{L}$ are the high and low equilibrium concentrations on the binodal curve, respectively and $c_{o}$ is the initial average concentration of the polymer solution $[2,3] .\left(t_{1}<t_{2}<t_{3}\right)$ 

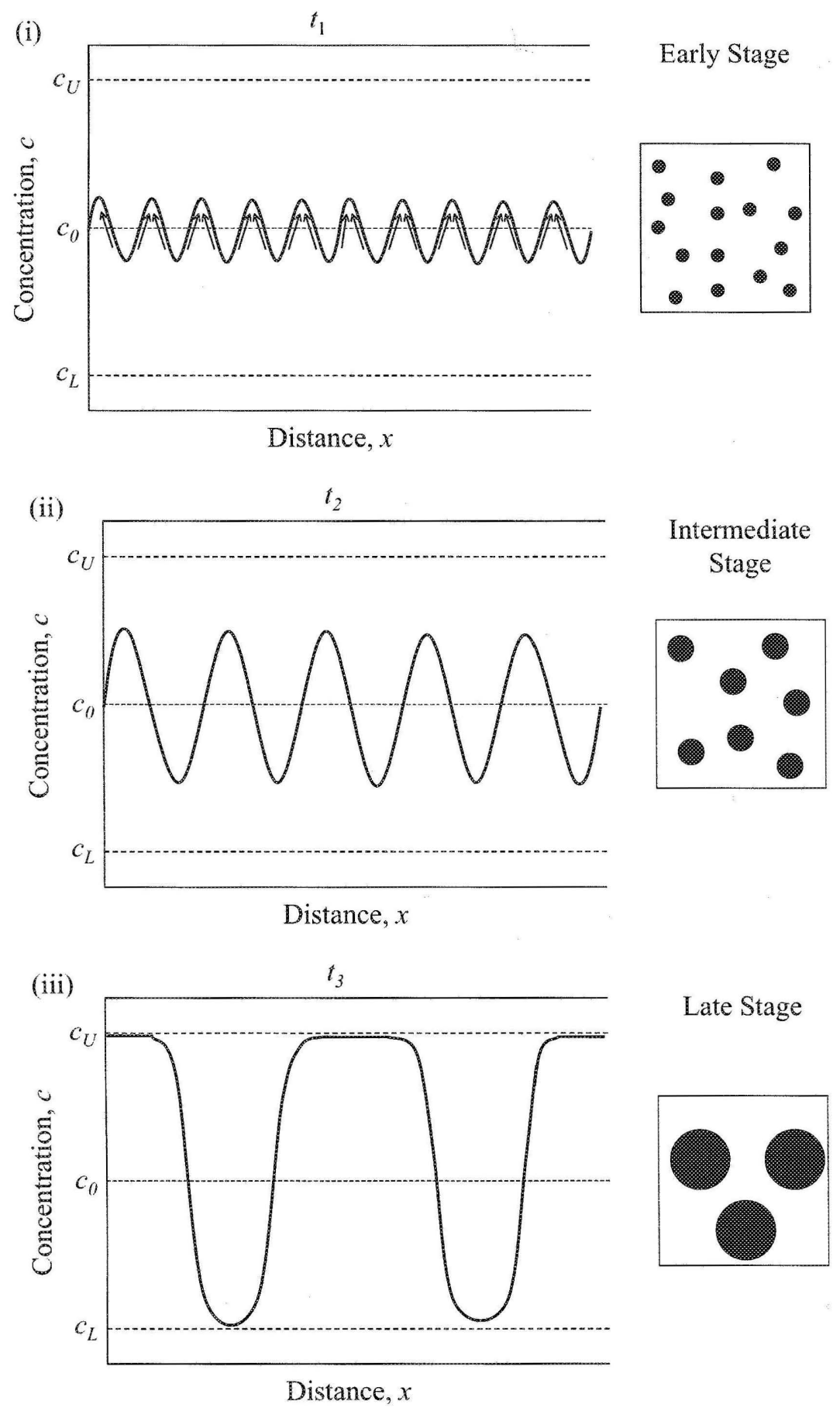

Figure 1.4: A schematic of one-dimensional spatial concentration profile of the phase separation by the Spinodal Decomposition (SD) mechanism from the initial concentration fluctuations at time, $t_{1}$ to the development and growth of domain sizes at time, $t_{3} . c_{U}$ and $c_{L}$ are the high and low equilibrium concentrations on the binodal curve, respectively and $c_{o}$ is the initial average concentration of the polymer solution $[1,2,3] .\left(t_{1}<t_{2}<t_{3}\right)$ 


\subsection{Methods of Phase Separation}

There are several methods of polymer phase separation available for manufacturing of the structural anisotropic materials, i.e. PDLC systems, microporous polymeric membranes etc. Out of these methods, Thermal-Induced Phase Separation (TIPS) and PolymerizationInduced Phase Separation (PIPS) methods are the most commonly used methods industrially for the formation of above mentioned materials [27] and therefore, these two methods will be described. Other than these methods, the Solvent-Induced Phase Separation (SIPS) method [27] is also used for the preparation of the above mentioned polymeric composites materials.

\subsubsection{Thermal-Induced Phase Separation (TIPS) Method}

PDLC films and microporous synthetic (polymeric) membranes are produced by this method, which relies on thermally driven phase separation process called Thermal-Induced Phase Separation (TIPS) [27]. In the TIPS methods, a polymer solution is rapidly cooled (quenched) (in case of the UCST solutions) or heated (in case of LCST solutions) in such a way that the conditions of the polymer solution falls in the spinodal region of the phase diagram from its initial homogeneous single phase condition, and consequently, it causes the phase separated regions to form within the solution. Polymer solutions undergoing TIPS via the spinodal decomposition (SD) mechanism may develop a droplet-type morphology $\left(c_{0}<c_{c}\right.$ and $\left.c_{0}>c_{c}\right)$ or interconnected-type structure $\left(c_{0}=c_{c}\right)$ depending on the initial concentration, $\left(c_{0}\right)[28,4]$. Critical (off-critical) initial concentrations result in the interconnected-type structure (droplet-type morphology). Functional polymeric materials normally require the droplet-type morphology that develops and evolves during the early and intermediate stages of SD before significant droplet coarsening occurs in the late stage of SD. Application of a temperature gradient [22] or a concentration gradient [19] causes an anisotropic droplet-type morphology to form, where the droplet size varies along the gradi- 
ent direction, but is uniform within the plane normal to the gradient direction. Therefore, it is important to have an understanding of the morphological development and evolution during the thermal-induced phase separation (TIPS) method by the SD mechanism in polymer systems in order to control the manufacturing process of functional polymeric materials, since product performance largely depends on the morphology and droplet size in the polymeric materials.

Figure 1.5 illustrates the TIPS method of phase separation by the SD mechanism in a polymer solution. Selection of the quench temperature, $T_{f}$ and initial average concentration, $c_{0}$ of the polymer solution depends on the end use of the polymeric phase separated micro-structures to be formed. As shown in Figure 1.5, three distinct routes or process paths of the TIPS method via the SD mechanism describe two different resultant morphologies: the droplet-type morphologies and the interconnected-type structures. In the case of the critical quench (route $B$ in which initial average concentration, $c_{0}$ is chosen to be the the critical concentration, $c_{c}$ of the solution in the phase diagram.), the resultant morphology is of interconnected-type structures, and in the case of the off-critical quench (route $\mathrm{A}$ or $\mathrm{C}$ in which initial average concentration of the solution, $c_{0}$ is chosen to be less than or greater than the critical concentration, $c_{c}$ of the solution, respectively.), the droplet-type morphology results [4]. The black regions represent the solvent-rich regions and the white regions represent the polymer-rich regions. The TIPS method via the SD mechanism, following the route $\mathrm{A}$, is the most widely and extensively used for the manufacturing of the functional polymeric materials, such as PDLC and microporous synthetic membranes industrially. Other process routes have also been studied during the course of this research thesis. 


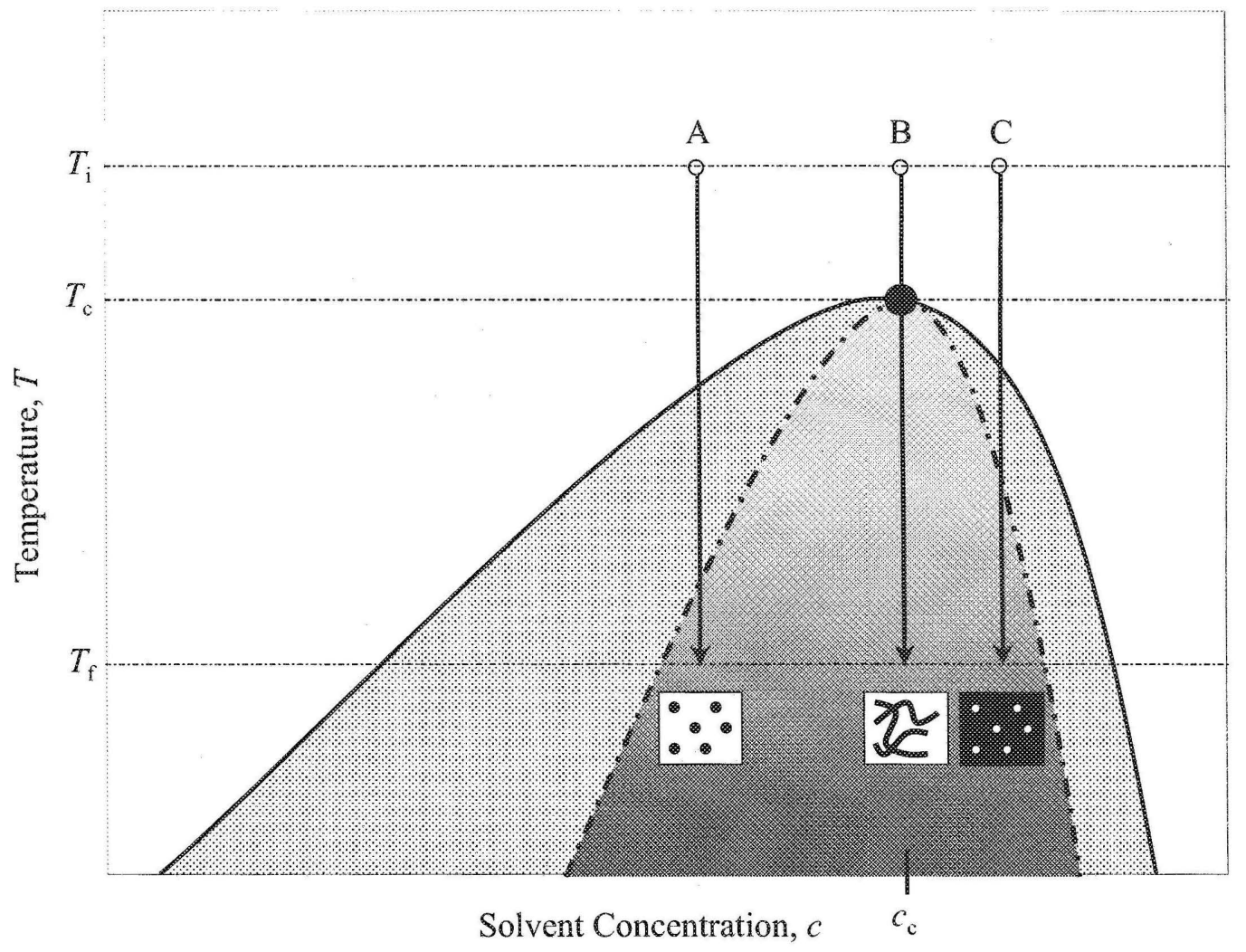

Figure 1.5: A schematic of the phase diagram showing the phase separation by TIPS method via the SD mechanism, which involves the quenching of a homogeneous single phase polymer solution at an average initial concentration, $c_{0}$ (open circles) from its initial temperature, $T_{i}$ to the final temperature, $T_{f}$. In the case of the critical quench (route $B$ ), the resultant morphology is of interconnected-type structures, and in the case of the off-critical quench (route $\mathrm{A}$ or $\mathrm{C}$ ), the droplet type morphology results. $c_{c}$ represents the critical concentration of the polymer solution [2].

\subsubsection{Polymerization-Induced Phase Separation (PIPS) method}

The polymerization-induced phase separation (PIPS) method has proved to be the most useful in forming the durable films with good electro-optical properties, though it is more complex process than the conventional thermal-induced phase separation (TIPS) method as it involves both the process of phase separation or de-mixing as well as the process of polymerization simultaneously $[11,29]$. Unlike the TIPS method, the PIPS method is usually irreversible as the crosslinking between the polymer chains is easily accomplished. 
Because of this crosslinking, the polymer matrix in the resulting film can be quite durable, leading to an excellent stability of the PDLC device properties. It is usually quite easy to change the chemical nature of the matrix in PIPS systems. The inherent flexibility in the polymerization process makes a wide variety of starting materials both possible and readily available. In PIPS methods, as the name implies, the phase separation is induced by converting the reactive monomer into the polymer through the process of polymerization.

In the PIPS method, first of all, a homogeneous single phase solution is prepared by mixing reactive monomers and non-reactive solvent at an initial average concentration, $c_{0}$ and initial temperature, $T_{i}$. This point on the phase diagram is called the cure point (black dot in Figure 1.6). Thus, the PIPS process starts with stable single-phase homogeneous mixture. The reactive monomers are then allowed to polymerize either by photo-initiation or thermal-initiation process. As the polymerization proceeds, the molecular weight of the polymer increases along with the solute degree of polymerization. As a result, the equilibrium curves, i.e. binodal and spinodal curves, of the polymer on the phase diagram shifts towards the higher solvent concentration and temperature (refer to Figure 1.6). One concern regarding PIPS systems is that it can be tricky to achieve uniform properties across a cell, or create many devices with exactly same properties.

The phase separation process is sensitive to temperature, light intensity (in photo-cured systems), the presence of impurities, the solubility characteristics and molecular weight of the starting materials. Variations in the phase separation process can lead to different matrix morphologies, which in turn affect the performance of the functional polymeric materials. Great care must be taken in PIPS systems to achieve reproducible and uniform devices. 


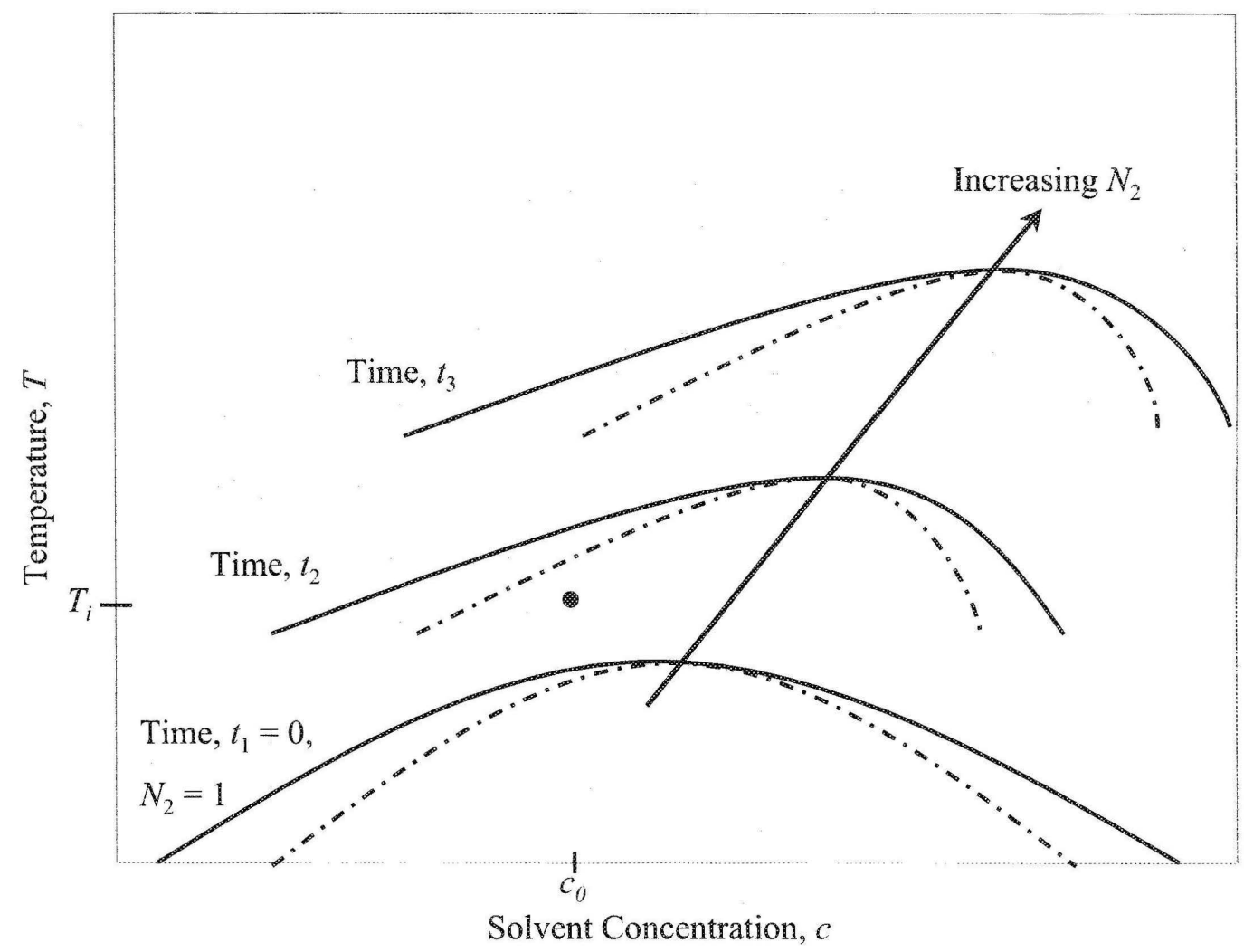

Figure 1.6: A typical phase diagram showing characteristic properties of the PIPS method. As the solute (polymer) degree of polymerization, $N_{2}$ increases, the phase diagram shifts upward (in the direction of the arrow): The phase separation begins as soon as the cure point (black dot) enters the unstable region or spinodal region of the phase diagram [4,2]. $\left(t_{1}<t_{2}<t_{3}\right)$

\subsection{The Phenomenon of Ludwig-Soret Effect}

A temperature gradient applied to a liquid mixture not only causes a heat flux, but also gives rise to a diffusion current of the constituent components. The resulting separation of the components causes the formation of a concentration gradient parallel or antiparallel with respect to the temperature gradient. This cross-effect between temperature and concentration is known as Ludwig-Soret effect or thermal diffusion [30,31]. Compared to other transport properties, thermal diffusion has not been much studied by computer simulations for TIPS methods of phase separation in polymer solutions under temperature 
gradient. During the last decade, however, a couple of different simulation methods have been applied to a few systems, aiming both at determination of numerical values of the Soret coefficient and at a better understanding of the phenomenon. Since its discovery by Ludwig (1856) and the first systematic investigations in liquid mixture by Soret (1879), the effect has been subject to experimental and theoretical studies. They observed a direct thermodiffusion in which a difference of a solute concentration (and thus, the matter flow) is caused by a temperature gradient. In particular, they found that the change in concentration occurs in a tube, which is filled with an initially homogeneous salt solution and has both ends kept at different temperatures. For instance, they showed that for a solution of sodium chloride $(\mathrm{NaCl})$, the salt concentration at the heated end of tube decreases, while at the cold end, it increases [31]. Furthermore, the effect is widely used in several industrial processes, such as separation of isotopes, polymer characterization, separation of products in a porous catalyst in chemical reactors, condensing a vapour mixture on a cold surface, and water injection in oil reservoir. All of these industrial processes have in common that they involve a mixture subject to a temperature gradient. This research thesis attempts to investigate and understand the effect of the thermal diffusion on the overall TIPS method of phase separation in a binary polymer solution under the influence of the externally imposed temperature gradient across the sample numerically and analyzes the morphological and statistical aspects of the polymer phase separation. 


\section{CHAPTER 2}

\section{LITERATURE REVIEW}

This chapter provides the literature review on the subject of the polymer phase separation in regards to its industrial applications and also in regards to the methods used to carry out the polymer phase separation process. This research work is limited to polymer phase separation method of thermal-induced phase separation (TIPS) via the SD mechanism, as it is one of the most widely used methods for the formation of many functional polymeric materials, such as polymer dispersed liquid crystal (PDLC) films, microporous polymeric membranes \& foams etc. This chapter also presents the literature review on the subject of thermal diffusion phenomenon in polymer solutions under a non-uniform temperature field.

\subsection{Thermal-Induced Phase Separation Method}

Thermal-induced phase separation (TIPS) has been the most widely used method for formation of the polymeric anisotropic functional materials, such as polymer dispersed liquid crystal (PDLC) devices as well the microporous synthetic materials (i.e. membranes, foams etc) since its invention by Castro in early 1980s [32]. There have been several numerical studies carried out for the investigation of the thermally-induced phase separation (TIPS)

method of phase separation in polymer solutions, specially by Chan and Rey $[28,33]$ in the last decade. 
Lee et al [22] developed a two-dimensional mathematical model describing the thermalinduced phase separation (TIPS) phenomenon via the spinodal decomposition (SD) mechanism in polymer solutions using the non-linear Cahn-Hilliard theory and the Flory-Huggins theory under an externally imposed spatial temperature gradient. The numerical results, in form of the spatial concentration profiles and patterns, indicated that isotropic morphology resulted from a uniform quench (no temperature gradients), while anisotropic morphology formed in case of a non-uniform quench (in presence of temperature gradient). The morphological analysis of the shape factor showed that the formation of droplet shape was independent of the spatial temperature gradient. Tran et al $[34,35]$ presented the computational study of the morphology development and evolution during the thermal-induced phase separation phenomenon via SD mechanism for a symmetric polymer blend in order to understand and control the morphology formation and evolution in the polymer blends to fabricate functional polymeric composite materials with predefined material properties and characteristics. The non-linear Cahn-Hilliard and the Flory-Huggins theories were used to model the TIPS method of phase separation via SD mechanism. Their simulation results indicated that the maximum light-scattering intensity increased exponentially during the early stage and early part of the intermediate stage and then slowed down during the later part of intermediate stage and late stage of the SD through the structure factor calculations. The simulation results also showed that the dimensionless diffusion coefficient might be manipulated to control the formation and evolution of the morphology during the SD to customize the functional polymeric materials' properties and characteristics. Jiang and Chan [19] presented the numerical results of mathematical model that described the two-dimensional TIPS phenomenon via SD mechanism in polymer solution. with linear concentration gradient (non-uniform quenches) and the comparison with the conventional TIPS process (uniform quench). Unlike the conventional TIPS process (uniform quench), the morphological development in the polymer solution was found to be a graded size distribution, i.e. anisotropic structures, for the case of non-uniform quench (in 
presence of linear initial concentration gradient) in their computational study. Apart from above referred general computational studies, there has also been significant amount of experimental as well as some numerical or computational studies performed in regards to the specific applications, i.e. formation of PDLC films, microporous synthetic membranes etc., which will be illustrated in following subsections.

\subsubsection{Polymer Dispersed Liquid Crystal (PDLC) Films}

Polymer-dispersed liquid crystal (PDLC) composites have been the subject of research for many years due to their applications in electro-optical devices, such as flat panel displays, switchable windows etc [11]. A PDLC film is composed of a polymer binder and a larger number of liquid crystal (LC) microdroplets or domains dispersed in it. Commonly used materials for the formation of PDLC films are polymethyl methacrylate (PMMA) and polyvinylformal (PVF) as polymeric matrix and 4-cyano-4-pentyl biphenyl (5CB) liquid crystals dispersed in it. A simple and the most common method for preparation of PDLC films is to exploit phase separation mechanism by the thermally-induced phase separation (TIPS) method. Thermally-induced phase separation (TIPS) process is useful in situations, where the polymeric malarial employed is readily melted. In this method, the liquid crystal is usually dissolved in a polymer melt, yielding a homogeneous solution. A thin film is then produced and material is cooled to induce phase separation followed by solidification. Other methods of phase separation for preparation of PDLC films include the solvent-induced phase separation (SIPS) and polymerization-induced phase separation (PIPS).

The pivotal work for studying the morphological control and structural analysis in preparation of PDLC film started in the late 1980s $[36,37,38]$. Since then, there has been a continuous research work going on in the area of modification and improvement of the properties of PDLC films for better performance. West [36] successfully studied and inves- 
tigated TIPS method of phase separation for preparation of PDLC films using the thermoplastic polymer-LC system of Epon 828 and $t$-butylamine. It was demonstrated that the rate of cooling of the polymer melts affected the resulting droplet morphology. Rapid cooling resulted in smaller diameter and more LC remaining in the binder, whereas the slower cooling resulted in larger droplets, which was because slower cooling allowed more time for phase separation, droplet growth and droplet coalescence. Under terrestrial conditions, gravitational forces can strongly influence the particle sizes and their distribution via sedimentation and coalescence, thereby making it difficult to study the importance of the intrinsic parameters, which control the process of TIPS for formation of PDLC films. Jin et al [39] analyzed the TIPS process and determined the critical parameters, which controlled the phase separation and the subsequent growth of the liquid crystal droplets in the PDLC systems under microgravity. In their study, the effect of cooling rate was investigated on droplet sizes in the TIPS process both experimentally and in simulation under microgravity environments, and found that, in both cases, a faster cooling rate yielded smaller droplets and improved the uniformity of their dispersions in the polymer binder. However, the simulation results indicated that surface interaction energy played a major role in setting up surface barriers, which hindered the growth. Their simulations results were found be in good agreement with the experimental results as far as final droplet sizes were concerned. Teixeira and Mulder [40] developed a model of polymer-dispersed liquid crystal (PDLC) formation by the thermally-induced phase separation (TIPS) via spinodal decomposition (SD) mechanism for the system consisted of thermoplastic polymer-LC mixture using the cell dynamical systems (CDS) method of Oono and Puri, suitably modified to describe a continuous temperature quench. Like others, the final morphology of PDLC was found to be dependent on the quench rate. Chan [4] modelled and simulated the formation of PDLC films via TIPS as well as PIPS method of phase separation via the SD mechanism using the non-linear Cahn-Hilliard (C-H) theory and the Flory-Huggins (F-H) theory. The numerical method of Galerkin finite element was used to solve the linearized 
fourth-order Cahn-Hilliard (C-H) equation in his study. The simulation results indicated that the droplet size decreased with increasing the diffusion coefficient, and eventually resulted in higher droplet density, whereas the droplet size increased with decreasing the diffusion coefficient and eventually resulted in lesser droplet density for the TIPS method of phase separation. Similarly, a higher quench (shallow quench) temperature resulted in smaller droplet size and higher droplet density, whereas a lower quench temperature (deep quench) resulted in bigger droplet size and corresponding lower droplet density. From these simulation findings, it was concluded that the droplet size and density of the PDLC composites were dependent on the diffusion coefficient and quench temperature of the phase separating solution or system. Whitehead and Gill [41] experimentally studied and investigated the formation of PDLC films via TIPS method using scanning electron microscopy (SEM) for a system consisted of thiol-ene based pre-polymer and LC components such as 4-pentyl-4-cyanobiphenyl (K15), 4-heptyl-4-cyanobiphenyl (K21) and 4-octyloxy-4cyanobiphenyl (M24). Their SEM results indicated that PDLC droplet size decreased with increasing cure temperature above the phase separation temperature for the respective liquid crystal pre-polymer mixture, whereas the PDLC droplet size increased with increasing liquid crystal composition in the liquid crystal pre-polymer mixtures cured at a constant temperature above the thermal phase separation temperature. Based on these findings, it was concluded that a careful choice of cure temperature and the amount of liquid crystal were required to manipulate the PDLC morphology and thus, PDLC electro-optical properties.

\subsubsection{Microporous Synthetic Membranes}

Microporous synthetic membranes are prepared by a variety of methods, including sintering of ceramic, graphite, metal or crystalline polymer powders; stretching of extruded homogeneous polyolefin or polytetrafluoroethylene (PTFE) films; track etching of homo- 
geneous polycarbonate or polyester films; and phase inversion solution casting of variety of polymers [42]. While each of these membrane preparation methods have their own advantages, the thermally-induced phase separation (TIPS) method is perhaps the most versatile and the simplest one for formation of the microporous polymeric materials, such as membranes, foams etc $[43,44]$. TIPS has been used for formation of the microporous polymeric membranes of controlled pore characteristics from a variety of crystalline and thermoplastic polymers, including polyolefins, condensation and oxidation polymers, copolymers, and blends $[32,20,21,45]$. Commercially available TIPS polypropylene membranes have proved useful in cross-flow microfiltration $[46,47]$ and plasmapheresis [48] and also have been investigated for possible use in membrane distillation [49]. Other uses of microporous TIPS membranes include separators in electrochemical cells, synthetic leather, "breathable" rainwear and diapers, as well as surgical dressings and bandages.

In its simplest form, the TIPS process is made up of following steps:

1. A single phase homogeneous polymer solution is formed by melt-blending a polymer with a high boiling, low molecular weight liquid or solid referred to as diluent (solvent).

2. The solution is then cast into the desired shape.

3. The cast solution is cooled to induced the phase separation and solidification of the polymer.

4. The solvent is then removed either by solvent evaporation process or solvent extraction process to produce a microporous structure.

TIPS method is distinguished from the familiar non-solvent-induced phase inversion method of membrane preparation in at least one important aspect. In TIPS method, the single phase homogeneous solution, from which the membrane is formed, is converted to a two 
phase mixture via the removal of thermal energy rather than by the slower exchange of non-solvent for solvent. TIPS method of microporous membrane preparation has several advantages over other methods of membrane preparation, which include the following:

- The TIPS process is applicable to a wide range of polymers, including the polymers that, because of poor solubility, could not be formed into membranes via the traditional non-solvent-induced phase inversion. In this regards, one of the distinct advantages of TIPS method is its ability to prepare membranes from semi-crystalline polymers.

- TIPS method is capable of producing a variety of microstructures $[42,50,32,45,20,21]$ and because of its versatility, it is the most popular and useful methods for the formation of variety of microstructural materials.

- TIPS method is capable of producing relatively thick isotropic microporous structures suitable for controlled release. Formation of anisotropic microporous structure is also possible, if a thermal gradient is induced in step 3 of the membrane preparation procedure outline above $[20,21]$.

- As the phase separation by TIPS method is thermally induced rather than nonsolvent exchange induced, it involves fewer variables, which need to be controlled, and as a result, the desired microporous membranes with a controlled characteristics can be produced with a great ease.

Following the four step procedure outlined above, the microporous polymeric membranes can be formed via solid-solid phase separation or via liquid-liquid phase separation with subsequent solidification of the polymeric system. There has been a great amount of experimental research work carried out for the betterment of the properties of these microporous polymeric membranes formed by TIPS method for last two decades or so. 
Lloyd et al [50] studied the formation of microporous crystalline polymer membranes via thermally-induced solid-liquid phase separation of polymer-solvent mixture experimentally. In their work, the thermally-induced solid-liquid phase separation process was studied in terms of the solution thermodynamics of binary mixture and the crystallization kinetics. The polymeric materials, investigated by them for the membrane formation, were polypropylene, high density polyethylene, polychlorotrifluoroethylene, poly(4methyl-1-pentene) and poly(vinylidene fluoride) etc. Lloyd et al [51] also studied microporous membrane formation via thermally-induced liquid-liquid phase separation of isotactic polypropylene- $n, n$-bis (2-hydroxyethyl) tallowmine mixture experimentally, of course and in their work, they successfully demonstrated that microporous membranes could be produced by liquid-liquid phase separation followed by solidification of the polymer or by solid-liquid phase separation. Kim et al [52] examined the role of diluent mobility and crystallization temperature in the formation of synthetic membranes via thermally-induced solid-liquid phase separation method in the systems of isotactic polypropylene/ $n$-alkane and isotactic polypropylene/ $n$-fatty acid systems. Kim and Lloyd [53] also experimentally investigated the effect of thermodynamic interactions on the structure of isotactic polypropylene membranes. On the other hand, Chiang and Lloyd [54] investigated the effect of dissolution temperature and polymer concentration on the nucleation density and subsequently, on the structure of membranes made by the solid-liquid TIPS process. They realized that the phase separation proceeded via the nucleation and growth (NG) mechanism to yield the droplet-type morphology (common in NG quench), when the rate of cooling was slow. On the other hand, they also observed the phase separation to proceed via the spinodal decomposition (SD) mechanism, when the rate of cooling was faster and obtained the interconnected-type structure for critical quench of the polymer solution (typical of SD quench).

There has also been several experimental studies carried out for the investigation of the 
effect of various process parameters on the properties and structure of the microporous membranes formation by the liquid-liquid TIPS process. Graham and McHugh [55] studied the kinetics of liquid-liquid TIPS method of phase separation and crystallization during thermal quenching of a polypropylene copolymer in anisole solution using small angle light scattering (SALS), differential scanning calorimetry (DSC) and scanning electron microscopy (SEM), and they realized the cessation of domain growth, when the solution temperature crossed the crystallization temperature. Matsuyama et al $[56,16]$ studied the effect of the cooling rate and the quench temperature on the membrane structures formed via liquid-liquid TIPS method and successfully created anisotropic or asymmetric membrane structures for the system of isotactic polypropylene (iPP) and diphenyl ether (DPE) by allowing the melted solution to evaporate from one side, thereby creating a polymer concentration gradient in the sample before the cooling or quenching. These kinds of the anisotropic membranes structures, with gradation in pore size, are highly desirable for the some microfiltration (MF) and/or ultrafiltration (UF) application. Compared with isotropic membranes with similar retention, anisotropic membranes show significant improvement in permeability and throughput. In a further study of the properties and structural control of microporous membrane, Matsuyama et al $[56,17,57,58,59]$ also studied the liquid-liquid as well as solid-liquid TIPS method of membrane formation for various polymeric systems in order to investigate the effect of various parameters, such as polymer molecular weight [58], diluent [57] and diluent evaporation rate or cooling rate $[56,17]$, extraction and drying [59].

Other than above mentioned experimental studies, many numerical studies have also been performed for the investigation of the formation and structural analysis of the microporous polymeric membranes. Many of these studies have revealed the fact that the spinodal decomposition (SD) mechanism plays the most important role in the TIPS method of phase separation during the formation of the microporous membranes. Caneba and Soong [21] 
were perhaps the first ones who modelled and simulated the conventional thermal inversion method of liquid-liquid phase separation for formation of the anisotropic membranes using the Flory-Huggins (F-H) theory for the polymer solution thermodynamics and the linearized Cahn-Hilliard theory for the spinodal decomposition (SD) mechanism. In their findings, it was observed that the growth period was relatively short compared with induction and coarsening times. In addition, it was also realized that the induction period increased with the phase separation temperature. They concluded from their modelling and simulation work that the linear theory could adequately predict the relevant pore sizes of thermal-cast membranes.

On the other hand, Barton et al [60] used a model based on the non-linear Cahn-Hilliard theory to simulate the structure formation in thermally quenched polymer solution in the vicinity of a glass transition, which was found to be in excellent agreement with their experimental work done on the phase separation dynamics in the PMMA/cyclohexanol system. It was concluded that the non-linear Cahn-Hilliard theory for SD mechanism could be employed for prediction of the microporous structural development during the early stage as well as the late stage of SD. Barton and McHugh [61] also portrayed the used of droplet growth rate data, obtained from the light scattering measurements and/or spinodal decomposition calculations, to model the evolution of pore size gradients in solutions undergoing temperature-induced phase inversion and demonstrated the effects of quench temperature on the transient and final pore size distribution in the quenched films (membranes). Graham et al [62] used the small-angle light scattering (SALS) and scanning electron microscopy (SEM) to study the morphology development in PMMA membranes formed by the TIPS method of phase separation. Their numerical analysis of the early time behavior of the scattered intensity in terms of Cahn-Hilliard theory established the spinodal decomposition (SD) mechanism as the mechanism of phase separation for the conditions studied. Matsuyama et al [63] performed kinetic studies of thermally-induced 
phase separation (TIPS) method of microporous membranes formation for polymer-diluent system composed of polypropylene/methyl salicylate. Their results showed a good fit with the linear Cahn-Hilliard theory of the spinodal decomposition (SD) mechanism.

\subsection{Thermal Diffusion Phenomenon in Polymers}

Isothermal static and dynamical properties of polymer blends/solution have been all well established $[8,64]$, but not many non-isothermal studies on phase separating polymers in the presence of a non-uniform temperature field have been carried out so far. Although, there have been some experimental $[65,66,67]$ as well as numerical $[68,22]$ studies reported in the literature on the phase separating polymer solutions and/or polymer blends under the influence of externally applied temperature gradient in recent times. None of these studies have taken into account the Ludwig-Soret effect, which leads to the mass flux as a result of externally applied temperature gradient across the polymer solution/blend.

Krekhov et al $[69,70]$ theoretically investigated the phase separation phenomena for polymer blends by modeling and simulating the coupled equations of heat and the extended Cahn-Hilliard equation incorporating the Ludwig-Soret effect or thermal diffusion phenomenon in presence of spatial periodic temperature modulations for the critical polymer blend/mixture. Their simulations produced a periodic kink-type patterns formation in the polymer sample domain. It was also determined, in one-dimension as well as twodimensions, the critical forcing amplitude necessary to produce a periodic stripe pattern from small random initial conditions, which depended weakly on wave number. It was also demonstrated from their experimental work that long-living spatial variation patterns with lifetimes of many hours could be written into polymer blend in the vicinity of its critical point by local laser heating. Reasonable agreement was demonstrated between their experimental and theoretical work. Enge and Kohler $[71,72]$ employed a holographic grating 
technique for the measurement of diffusion and, for the first time, thermal diffusion properties of a critical polymer blend of poly(dimethyl siloxane) (PDMS) and poly(ethylmethyl siloxane) (PEMS). Experimental results showed that the Ludwig-Soret effect played an essential role in phase separating polymer systems subjected to temperature gradient. It was also concluded from their work that even small temperature differences could cause substantial composition changes, which could not be neglected in the treatment of nonisothermal phase separation. Rauch and Kohler [73,74] investigated mass and thermal diffusion in polystyrene (PS)/toluene solutions over the whole concentration range from dilute to concentrated. The collective mass diffusion, thermal diffusion and Soret coefficients were determined for the PS/toluene solution by a transient holographic grating technique and photon correlation spectroscopy. It was realized that the Soret coefficient, which was given by ratio of thermal diffusion to collective mass diffusion, was insensitive to the glass transition and followed the scaling law in the semi-dilute to concentrated polymer solutions without glass transition.

de Gans et al [75] presented the results on the thermodiffusion of poly(ethylene oxide) (PEO) in ethanol/water mixture using the Thermal Diffusion Forced Rayleigh Scattering (TDFRS) technique. In these TDFRS experiments, the PEO was found to migrate towards the region of lower temperature in water-rich solvent mixture. This is typical for polymer solutions and corresponds to a positive Soret coefficient of PEO. In solvent-rich mixtures, however, the polymer was found to migrate towards the higher temperatures, corresponding to a negative Soret coefficient of a polymer in solution. Thus, from these experimental findings, polymer molecules were found to move into the direction of the thermodynamically favored preferentially solvating solvent shell composition. A simple lattice model for the polymer solvent system was presented and Soret coefficients were calculated with statistical mechanics methods, which were found to be in good agreement with their experimental results. 


\subsection{Thesis Objectives}

As mentioned earlier, our previous work by Lee et al numerically studied the TIPS method of phase separation in polymer solutions under temperature gradient [22]. In their study, the thermal diffusion phenomenon was not taken into consideration. Some researchers around the world pointed out that the Ludwig-Soret effect or the thermal diffusion phenomenon could not be neglected while studying polymer solutions under a temperature gradient, as it also contributes to the overall phase separation mechanism in polymer solutions. Therefore, the main objective of this research thesis is to study the Ludwig-Soret effect (Thermal Diffusion) on the thermal-induced phase separation (TIPS) method via the SD mechanism in polymer solutions with an externally imposed spatial temperature gradient through mathematical modelling and computer simulations. An emphasis has been placed specifically on the understanding of the influence of the thermal-diffusion phenomenon on the overall process of phase separation (TIPS in this case) under the spinodal or unstable region of the phase diagram in a binary polymer solution. The sequential objectives of the research thesis are as follow:

1. To develop a mathematical model (one dimensional as well as two dimensional), which describes the overall process of phase separation (i.e. TIPS via SD only) in a binary polymer solution sample involving thermal diffusion phenomenon (LudwigSoret effect) under an externally imposed spatial linear temperature gradient. The binary polymer solution sample is well suited for the TIPS method, as it is one of the most commonly used methods for the fabrication of functional anisotropic polymeric materials, i.e. PDLC films, micro-porous synthetic membranes etc. The theories, which will be taken into account during the development of the mathematical model for the research thesis, are the non-linear Cahn-Hilliard theory describing the TIPS method via the SD mechanism, Flory-Huggins theory describing the thermodynamics of polymer solutions, and theory of Ludwig-Soret effect describing the thermal diffu- 
sion phenomenon prevailing in a polymer solution under a non-uniform temperature field.

2. To implement, solve and validate a one-dimensional model as specified and developed in the above mentioned objective 1 for the TIPS method involving the thermal diffusion phenomenon. An emphasis is placed on the understanding of the influence of the thermal diffusion phenomenon on the overall phase separation mechanism (TIPS via SD) in binary polymer solutions under an externally imposed spatial linear temperature gradient by comparing the spatial structural profile (i.e. spatial concentration profile), obtained with and without thermal diffusion phenomenon.

3. To implement, solve and validate a two-dimensional model as specified and developed in the above mentioned objective 1 for the TIPS method involving the thermal diffusion phenomenon. Again, an emphasis is placed on the understanding of the influence of the thermal diffusion phenomenon on the overall phase separation mechanism (TIPS via SD) in binary polymer solutions under an externally imposed spatial linear temperature gradient by analyzing the structural morphologies obtained with and without thermal diffusion phenomenon. 


\section{CHAPTER 3}

\section{MODEL DEVELOPMENT}

This chapter presents the theoretical background for development of mathematical model of the phenomena of TIPS method of phase separation by the spinodal decomposition (SD) mechanism involving thermal diffusion phenomenon under the influence of an externally imposed spatial linear temperature gradient. As mentioned in the previous chapter, the TIPS method of phase separation by the spinodal decomposition (SD) mechanism is the most widely used mechanism for the fabrication of the functional polymeric materials, such as PDLC and microporous synthetic membrane. This research thesis primarily concentrates on the TIPS method of phase separation by the spinodal decomposition (SD) mechanism. Spinodal decomposition (SD) mechanism is best described by the well-known non-linear Cahn-Hilliard $(\mathrm{C}-\mathrm{H})$ theory, which will be described later in the chapter. This chapter also describes the theory of Ludwig-Soret effect, as it best describes the thermal diffusion phenomenon prevailing during the TIPS method of phase separation in a polymer solution under the influence of an externally imposed temperature gradient.

\subsection{Thermodynamics in Polymer Solutions}

As mentioned earlier, the understanding of the thermodynamics in polymer solutions is very vital in order to study the phase equilibria, and consequently, the phase separation phenomena in binary polymer solutions, especially under the influence of externally applied temperature gradient across the sample. The phase equilibria of a binary polymer 
solution can be very well described by the Flory-Huggins (F-H) theory. Therefore, the Flory-Huggins theory will be discussed in the chapter, as it describes the free energy of mixing for a binary polymer solution. And also, the construction of the phase diagram of a binary polymer solution using the Flory-Huggins theory will be illustrated later in the chapter.

The fundamental thermodynamic equation to describe the systems, such as polymer mixture relates the Gibbs free energy of mixing, $\Delta G^{M}$ to the enthalpy of mixing, $\Delta H^{M}$ and the entropy of mixing, $\Delta S^{M}$ under constant temperature and pressure.

$$
\Delta G^{M}=\Delta H^{M}-T \Delta S^{M}
$$

A homogeneous solution is obtained, when the Gibbs free energy of mixing, $\Delta G^{M}<0$, i.e. when the Gibbs free energy of solution, $G_{12}$ is lower than the Gibbs free energies of the constituent components of the mixture in pure state, $G_{1}$ and $G_{2}$.

$$
\Delta G^{M}=G_{12}-\left(G_{1}+G_{2}\right)
$$

\subsubsection{The Flory-Huggins Theory}

One of the most well-known expression for the free energy of mixing, $\Delta G^{M}$ of a polymer solution is the Flory-Huggins (F-H) theory $[5,76]$, which is based on the two dimensional statistical lattice model. This lattice model is used to characterize the possible arrangements of the components' segments in the polymer mixtures. Polymer solutions are considered as the irregular nonideal solutions, in which both $\Delta H^{M}$ and $\Delta S^{M}$ deviate from their ideal values. To understand the behavior of polymers in solution more fully, the knowledge of enthalpic and entropic contributions to $\Delta G^{M}$ is essential. 
For a system in a given state, the entropic contributions, also referred to as the combinatorial contributions, are related to the number of distinguishable arrangements the components in that state can adopt. These entropic contributions can be expressed by following equation derived from the Boltzmann's law of entropy based on the two dimensional statistical lattice model for the polymer solutions as follows [5]:

$$
\Delta S^{M}=-\frac{k_{B}}{\nu}\left[\frac{c}{N_{1}} \ln c+\frac{(1-c)}{N_{2}} \ln (1-c)\right]
$$

where, $k_{B}$ is the Boltzmann's constant, $N_{1}$ and $N_{2}$ are the number of molecules or degree of polymerization of the component 1 (solvent) and 2 (solute), respectively; $c$ is the concentration of the component 1 (solvent in this case) in terms of its volume fraction in the mixture; and $\nu$ is the volume of the cell or segment.

The enthalpic contributions, also referred to as non-combinatorial contributions, are due to the interactions of polymer molecules with solvent molecules, and are much harder to quantify. The derivation of $\Delta S^{M}$ from the lattice theory has been made on the assumption that no heat or energy change occurs on mixing. This is an uncommon situation as experimental experience suggests that the energy change is finite. The theory of the regular solution is utilized in order to obtain the expression for $\Delta H^{M}$, where this change in energy is assumed to arise from the formation of new solvent-polymer interactions on mixing, which replace some of the solvent-solvent and polymer-polymer interactions present in the pure solvent and pure polymer components, respectively. Thus, based on the lattice model for the polymer solutions, the expression for the enthalpic contributions can be presented as follows [5]:

$$
\Delta H^{M}=\frac{k_{B} T}{\nu} \chi c(1-c)
$$

where, $T$ is the temperature, and $\chi$ is the Flory-Huggins interaction parameter, which is an important feature of the polymer solution theory. The Flory-Huggins interaction parameter 
is zero for athermal solutions, positive for endothermic and negative for exothermic mixing.

Now, combining Equations (3.3) and (3.4) into Equation (3.1) to give the expression for the Gibbs free energy of mixing, $\Delta G^{M}$ or the Flory-Huggins free energy, $f(c)$ as follows [5]:

$$
f(c)=\frac{k_{B} T}{\nu}\left[\frac{c}{N_{1}} \ln c+\frac{(1-c)}{N_{2}} \ln (1-c)+\chi c(1-c)\right]
$$

\subsubsection{Phase Equilibria and Phase Diagram}

The Flory-Huggins theory can be used to predict the equilibrium behavior of two liquid phases, when both contain amorphous polymer and one or two solvents. Consider a twocomponent system consisting of a component 1 (solvent) and component 2 (polymer). Complete miscibility occurs, when the Gibbs free energy of mixing, $\Delta G^{M}$ is less than the Gibbs free energies of the components, and the solution maintains its homogeneity only as long as $\Delta G^{M}$ remains less than the Gibbs free energy of any two possible coexisting phases.

Figure 3.1 represents a schematic diagram of the Gibbs free energy of mixing, $\Delta G^{M}$ as a function of the solvent concentration (top half), showing the transition from a system miscible in all proportions at a temperature, $T_{1}$ through the critical temperature, $T_{c}$ to partially miscible systems at temperatures, $T_{2}$ to $T_{4}$. The contact points for the common tangents drawn to the minima of the Gibbs free energy curve for temperature, $T_{4}$ are shown projected onto the temperature-concentration plane to form the binodal (cloud point) curve, whereas projection of the inflexion points forms the spinodal curve, and the area under the spinodal curve represents the heterogeneous two-phase region, where there is limited solubility of component 2 (polymer) in component 1 (solvent) and vice versa, depending on the location of the quench point $(c, T)[5]$. This is also called a cloud point curve. As the temperature is increased, the limit of this two-phase coexistence contracts, 


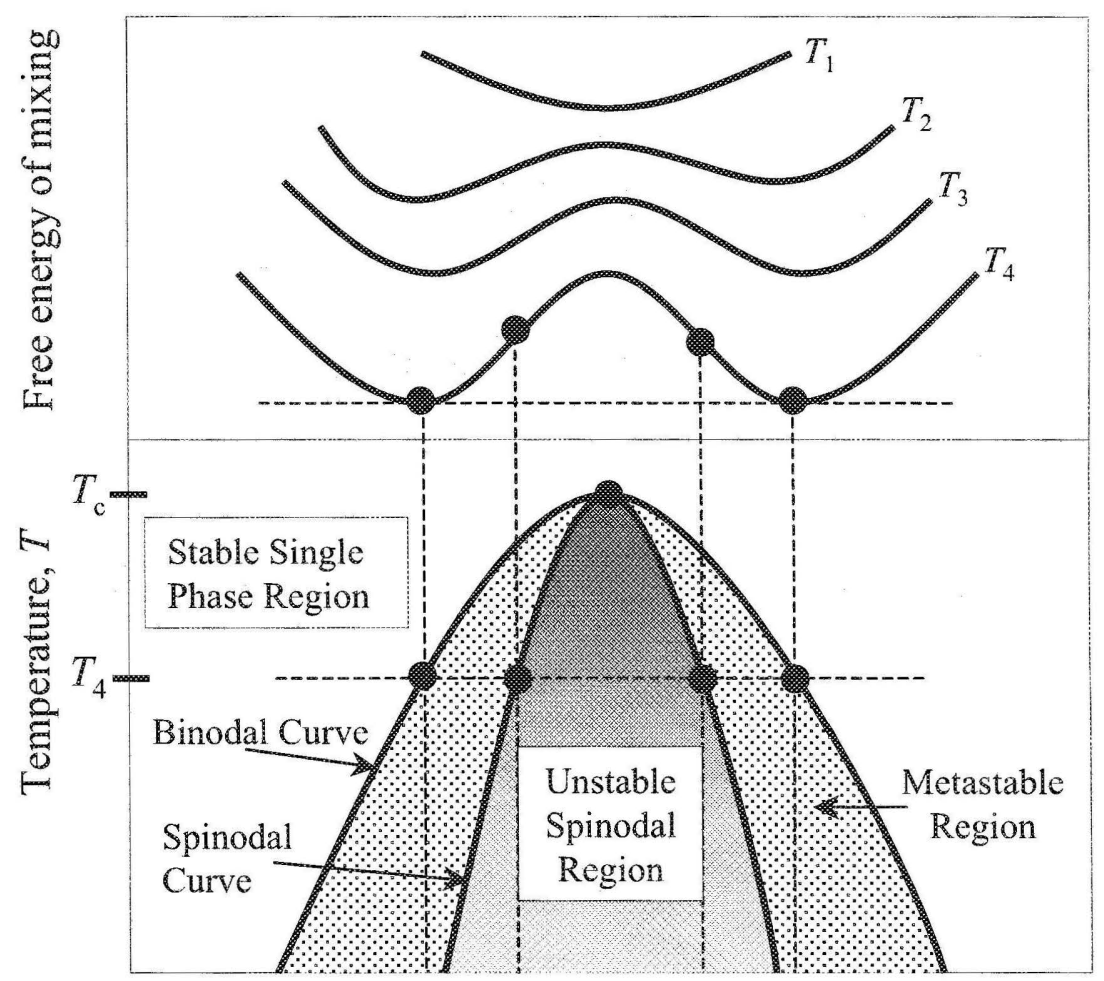

Concentration, $c$

Figure 3.1: A Schematic diagram of the Gibbs free energy of mixing as a function of the solvent concentration (top half) and typical schematic symmetrical phase diagram for a binary solution (bottom half) showing the metastable region (dotted area) and unstable spinodal region (gray shaded area). The contact points for the common tangents drawn to the minima are shown projected onto the temperature-concentration plane to form the binodal (cloud point) curve, whereas projection of the inflexion points forms the spinodal curve. The upper diagram shows the different Gibbs free energy curves corresponding to the temperatures $T_{1}$ to $T_{4}\left(T_{1}>T_{2}>T_{3}>T_{4}\right)$ [5].

until eventually, they coalesce to produce a homogeneous one phase mixture at $T_{c}$, the critical solution temperature. The critical solution temperature is an important quantity, and can be accurately defined in terms of the chemical potential. It represents the point at which the inflexion points on the curve merge, and so it is the temperature where the first, second and third derivatives of the Gibbs free energy with respect to the solvent concentration are zero. 
The phase diagram in Figure 3.1 can also be formed numerically using the Flory-Huggins Equation (3.5) [77]. In order to determine the equilibrium (binodal curve) composition, the Flory-Huggins (F-H) free energy Equation (3.5) and its first derivative with respect to composition are used to find the chemical potential of each component in their respective phases. Following are the equations of the chemical potential of each component in their respective phases [77]:

$$
\begin{gathered}
\Delta \mu_{1}^{\dagger}=\frac{k_{B} T}{\nu}\left[\ln c^{\dagger}+\left(1-\frac{1}{N_{1} N_{2}}\right)\left(1-c^{\dagger}\right)+\chi N_{1}\left(1-c^{\dagger}\right)^{2}\right] \\
\Delta \mu_{1}^{\dagger}=\frac{k_{B} T}{\nu}\left[\ln c^{\ddagger}+\left(1-\frac{1}{N_{1} N_{2}}\right)\left(1-c^{\ddagger}\right)+\chi N_{1}\left(1-c^{\ddagger}\right)^{2}\right] \\
\Delta \mu_{2}^{\dagger}=\frac{k_{B} T}{\nu}\left[\ln \left(1-c^{\dagger}\right)+\left(1-\frac{1}{N_{1} N_{2}}\right) c^{\dagger}+\chi N_{2} c^{\dagger^{2}}\right] \\
\Delta \mu_{2}^{\ddagger}=\frac{k_{B} T}{\nu}\left[\ln \left(1-c^{\ddagger}\right)+\left(1-\frac{1}{N_{1} N_{2}}\right) c^{\ddagger}+\chi N_{2} c^{\dagger^{2}}\right]
\end{gathered}
$$

Equations (3.6) to (3.9) give the chemical potentials of components 1 and 2 of the mixture in the two equilibrium phases (i.e. $\dagger$ and $\ddagger$ ). But, chemical potential is same everywhere in the two phases for components 1 and 2 (i.e. for component $1, \mu_{1}^{\dagger}=\mu_{1}^{+}$) at the equilibrium [77]. Therefore, following two equations are obtained by equating Equation (3.6) with (3.7) and (3.8) with (3.9),

$$
\begin{gathered}
\ln c^{\dagger}+\left(1-\frac{1}{N_{1} N_{2}}\right)\left(1-c^{\dagger}\right)+\chi N_{1}\left(1-c^{\dagger}\right)^{2}=\ln c^{\ddagger}+\left(1-\frac{1}{N_{1} N_{2}}\right)\left(1-c^{\ddagger}\right)+\chi N_{1}\left(1-c^{\ddagger}\right)^{2} \\
\ln \left(1-c^{\dagger}\right)+\left(1-\frac{1}{N_{1} N_{2}}\right) c^{\dagger}+\chi N_{2} c^{\dagger^{2}}=\ln \left(1-c^{\ddagger}\right)+\left(1-\frac{1}{N_{1} N_{2}}\right) c^{\dagger}+\chi N_{2} c^{\dagger^{2}}
\end{gathered}
$$

Now, these two Equations (3.10) and (3.11) are solved simultaneously for values of $c^{\dagger}$ and $c^{\ddagger}$ to construct the binodal curve, which represents the coexisting phases in equilibrium.

The spinodal curve is obtained by taking the second derivative of the Flory-Huggins (F-H) 
free energy equation and setting it equal to zero as shown below.

$$
(1-c)^{2}-\left(1-\frac{N_{2}-1}{2 \chi N_{2}}\right)(1-c)+\frac{1}{2 \chi N_{2}}=0
$$

Above equation is a quadratic equation, and is solved for values of composition, $c$ to construct the spinodal curve. Note that the Flory-Huggins interaction parameter, $\chi$ is needed in order to evaluate Equations (3.10) to (3.12). It is commonly considered to be the function of temperature and is expressed as follows $[5,77,76]$ :

$$
\chi=0.5-\psi\left(1-\frac{\theta}{T}\right)
$$

where, $\psi$ is the dimensionless entropy of dilution parameter, and $\theta$ is the theta temperature, which is defined as follows:

Theta temperature, $\theta$ of a dilute polymer solution is the temperature at which the polymer solution behaves as if it were an ideal solution [5].

The theta temperature, $\theta$ is a well-defined state of a polymer solution at which the excluded volume effects are eliminated, and the polymer coil is in an unperturbed condition. Above the theta temperature, expansion of the polymer coil takes place, caused by the interactions with solvent, whereas below $\theta$, the polymer segments attract one another, coils tend to collapse, and eventual phase separation occurs [5].

The intersection of the binodal curve and spinodal curve represents the critical point. It is calculated by setting the second derivative equal to the third derivative of the FloryHuggins free energy equation. The properties of the critical points are as shown below:

$$
c_{c}=1-\frac{1}{\left(1+\sqrt{N_{2}}\right)}
$$




$$
\begin{gathered}
\chi_{c}=\frac{1}{2}\left(1+\frac{1}{\sqrt{N_{2}}}\right)^{2} \\
\frac{1}{T_{c}}=\frac{1}{\theta}+\frac{1}{\theta \psi}\left(\frac{1}{\sqrt{N_{2}}}+\frac{1}{2 N_{2}}\right)
\end{gathered}
$$

where, $c_{c}$ represents the critical concentration of the solvent, $\chi_{c}$ is the critical value of Flory's interaction parameter, and $T_{c}$ is the critical temperature.

\subsection{The Cahn-Hilliard Theory}

The theory of phase separation in homogeneous single phase solution by spinodal decomposition was first given by J.W. Cahn back in 1956 [78]. Most of the studies for the kinetics of phase separation were limited to metals and glasses, but for last two decades or so, the study of the kinetics of phase separation has been extended to polymer solutions and blends [79] as well. The non-linear Cahn-Hilliard equation, describing the spinodal decomposition process, was derived from continuity equation of mass, which relates the inter-diffusional flux to the driving force of phase separation, i.e. the gradient in chemical potential.

The continuity equation is expressed as follows:

$$
\frac{\partial c}{\partial t}=-\nabla \cdot J
$$

where, $c$ is the concentration of solvent in terms of volume fraction, and $\mathbf{J}$ is the interdiffusional flux of the component (i.e. solvent). The kinetics of the initial stage of phase separation can be obtained simply by solving the diffusion equation, in which the interdiffusional flux is related to the gradient in chemical potential by following equation.

$$
\mathbf{J}=-M \nabla\left(\mu_{2}-\mu_{1}\right)
$$


where, $M$ is the mobility, which is defined by the ratio of the negative of inter-diffusional flux and the gradient in chemical potential. Mobility is considered to be the function of concentration, which will be discussed later in the chapter. $\mu_{1}$ and $\mu_{2}$ are the chemical potentials of the component 1 (solvent) and 2 (polymer) of the binary polymer solution, respectively. For simple thermodynamics considerations, mobility, $M$ must be positive if diffusion, which results spontaneously due to the gradient in chemical potential in the solution, is to result in decrease in the free energy of the system. Now, consider an inhomogeneous binary polymer solution, whose concentration, $c$ differs slightly from its average initial concentration, $c_{0}$ everywhere with infinitesimally small concentration gradients. Its total free energy of mixing can be given by following expression [78]:

$$
F=\int\left[f(c)+\kappa(\nabla c)^{2}\right] d V
$$

Here, $f(c)$ is the Flory-Huggins free energy density of homogeneous solution of concentration, $c$ whereas $\kappa(\nabla c)^{2}$ represents the changes in the free energy of the solution due to concentration gradients. $\kappa$ is a gradient energy coefficient, and it has been assumed to be a positive constant. The variational derivative of Equation (3.19) gives the chemical potential difference $\mu_{2}-\mu_{1}$ as expressed below [78].

$$
\mu_{2}-\mu_{1}=\frac{\delta F}{\delta c}=\frac{\partial f(c)}{\partial c}-2 \kappa \nabla^{2} c
$$

Combining Equations (3.18) and (3.20), we get the net inter-diffusional flux of the binary polymer solution related to the total free energy as expressed below:

$$
\mathbb{J}=-M \nabla\left[\frac{\partial f(c)}{\partial c}-2 \kappa \nabla^{2} c\right]
$$


Similarly, combining Equations (3.17) and (3.21), it gives non-linear Cahn-Hilliard equation as shown below:

$$
\frac{\partial c}{\partial t}=\nabla \cdot\left[M \nabla\left(\frac{\partial f(c)}{\partial c}-2 \kappa \nabla^{2} c\right)\right]
$$

At very early times, the concentration fluctuations are very small, and Equation (3.22) can be linearized about the average initial concentration, $c_{0}$ assuming constant $M$ and $\kappa$ resulting in following expression for $\mathrm{SD}[4]$.

$$
\frac{\partial c}{\partial t}=\nabla \cdot\left[\nabla\left(\left.M \frac{\partial f(c)}{\partial c}\right|_{c_{0}}-2 \kappa \nabla^{2} c\right)\right]
$$

The general solution of the above Equation (3.23) can be given by making use of the Fourier series as shown below [4]:

$$
\begin{gathered}
c(r, t)=c_{0}+\sum_{\mathbf{k}} A(\mathbf{k}, t) e^{i \mathbf{k} r} \\
A(\mathbf{k}, t)=A(\mathbf{k}, 0) e^{R(\mathbf{k}) t} \\
R(\mathbf{k})=-M \mathbf{k}^{2}\left[\left.\frac{\partial^{2} f(c)}{\partial c^{2}}\right|_{c_{0}}+2 \kappa \mathbf{k}^{2}\right]
\end{gathered}
$$

where, $\mathbf{k}$ is the wave vector, and is given by $\mathbf{k}=2 \pi / \lambda$ ( $\lambda$ is the wavelength for fluctuation); and $R(\mathbf{k})$ is the amplification factor. However, the linearized $\mathrm{C}-\mathrm{H}$ Equation (3.23) is not valid for intermediate and late stages of the phase separation by SD.

\subsection{Mutual and Self-Diffusion Coefficients}

Mutual diffusion process accounts for the relaxation of the thermal fluctuations in concentration of the binary solution. It describes the mixing of the components in the mixture 
through the mutual diffusion coefficient, $D$ given by following equation:

$$
D=Q \Lambda
$$

where, $Q$ is the thermodynamic factor related to the static correlation function of concentration fluctuations, and $\Lambda$ is a kinetic factor presumed to be expressible in terms of the self-diffusion coefficients of the components of the solution. The thermodynamic factor, $Q$ is expressed as follows [80]:

$$
Q=\frac{1}{N_{1} c}+\frac{1}{N_{2}(1-c)}
$$

where, $N_{1}$ and $N_{2}$ are the degree of polymerization of the component 1 and 2 in the binary solution, and $c$ and $(1-c)$ are the volume fractions of the component 1 and 2 in the binary solution. However, as far as the analytical form of $\Lambda$ is concerned, there exists two contradictory theories, namely slow mode theory and fast mode theory, which take into account the self-diffusion coefficients of the individual component of the solution.

The slow mode result is obtained with the random-phase approximation under the condition of microscopic incompressibility and on the other hand, the fast mode result is obtained by ignoring the dynamic correlations between the velocities of different particles [80]. Both these results are expressed as shown below:

$$
\begin{gathered}
\frac{1}{\Lambda_{\text {slow }}}=\frac{1}{N_{1} D_{1} c}+\frac{1}{N_{2} D_{2}(1-c)} \\
\Lambda_{\text {fast }}=c(1-c)\left\{N_{1} D_{1}(1-c)+N_{2} D_{2} c\right\}
\end{gathered}
$$

where, $D_{1}$ and $D_{2}$ are the respective self-diffusion coefficients of the components 1 and 2 in the binary polymer solution. Mutual or inter-diffusion coefficient corresponding to the slow mode theory and the fast mode theory can be expressed as following in terms of the 
self-diffusion coefficients [80].

Slow Mode: $\quad \frac{1}{D}=\left(\frac{1}{N_{1} D_{1} c}+\frac{1}{N_{2} D_{2}(1-c)}\right) /\left(\frac{1}{N_{1} c}+\frac{1}{N_{2}(1-c)}\right)$

Fast Mode: $\quad D=c(1-c)\left\{N_{1} D_{1}(1-c)+N_{2} D_{2} c\right\}\left(\frac{1}{N_{1} c}+\frac{1}{N_{2}(1-c)}\right)$

From Equations (3.31) and (3.32), we can see that, for the case where one species is diffusing much faster than the other (i.e. $D_{1} \gg D_{2}$ ), Equation (3.31) predicts that the interdiffusion is controlled by the slow diffusing component, i.e. $D \approx D_{2}\left[N_{2}(1-c) / N_{1} c+1\right]$ (slow mode theory), while Equation (3.32) predicts that the interdiffusion is controlled by the fast diffusing component in the mixture, i.e. $D \approx D_{1}(1-c)^{2}\left[1+N_{1} c / N_{2}(1-c)\right]$ (fast mode theory). Thus, the mutual diffusion or interdiffusion process is dependent upon the self-diffusion coefficients of the individual components of the solution. There are several models or theories available, which help in determination of the self-diffusion coefficients of the constituent components of the solution, i.e. the spring-and-bead model, the Rouse theory, the Kirkwood-Riseman theory and the reptation theory [81]. This research thesis makes use of the Rouse theory based on the spring-and-bead model to determine the selfdiffusion coefficient, as it best describes the slow diffusion mechanism behavior by the low molecular weight polymer solutions such as the ones considered in this work, and therefore, only Rouse theory will be described briefly.

Based on the spring-and-bead model, the Rouse theory assumes that there are no hydrodynamic interactions between the polymer segments, and therefore, the solvent molecules are able to move freely through the polymer coil. However, the presence of the solvent molecules in the polymer coils inhibits the movement of polymer segments, i.e. there exists the molecular friction. Moreover, this theory is applicable to the systems having short polymer chains with no entanglement effect, which is true for $N_{2}<200$. Keeping in mind all these assumptions, the self-diffusion coefficient, derived from the Rouse theory, can be 
expressed as follows $[81,82]$.

$$
D_{i}=\frac{k_{B} T}{N_{i} \xi_{i}} \quad \text { for } i=1,2
$$

where, $D_{i}$ is the self-diffusion coefficient of the component $i$, i.e. $D_{1}$ for solvent and $D_{2}$ for polymer, derived from the Rouse theory, $k_{B}$ is the Boltzmann's constant, $T$ is the temperature, $N_{i}$ is the degree of polymerization (or number of segments) of the $i^{t h}$ component in the solution, and $\xi_{i}$ is the frictional coefficient of the $i^{\text {th }}$ component per segment in the solution.

\subsection{Concentration Dependent Mobility}

The mobility is a measure of rate at which the concentration of the mixture is dispersed, and is known to be a function of concentration as mentioned earlier. The slow mode theory states that the mobility, $M$ is related to the self-mobilities of the constituent components, $M_{1}$ (i.e. for solvent) and $M_{2}$ (i.e. for polymer) as:

$$
\frac{1}{M}=\frac{1}{M_{1}}+\frac{1}{M_{2}}
$$

The individual mobility of the constituent components can be expressed in terms of the respective self-diffusion coefficient as:

$$
M_{i}=\frac{D_{i}(c)}{\left(\frac{\partial^{2} f(c)}{\partial c_{i}^{2}}\right)} \quad \text { for } i=1,2
$$

where, $c_{i}$ is the concentration of the component $i$, i.e. $c_{1}=c$ and $c_{2}=(1-c)$. Now, substituting the values of the respective self-diffusion coefficients from Equation (3.33) and the respective second derivatives of the Flory-Huggins $(\mathrm{F}-\mathrm{H})$ free energy equation from Equation (3.5) into Equation (3.35) to obtain the respective mobilities of the constituent 
components 1 (solvent) and 2 (polymer):

$$
\begin{gathered}
M_{1}=\frac{\nu c}{\xi_{1}} \\
M_{2}=\frac{\nu(1-c)}{\xi_{2}}
\end{gathered}
$$

The Rouse theory based on the spring-and-bead model, which describes the motion of the polymer chain as spring and bead, can be observed in the concentrated polymer solution $[81,83]$. Since, we are modeling concentrated solutions containing short polymer chains with no entanglements and hydrodynamics effects, the Rouse model may be used to express the self-diffusion coefficients. The following expression is obtained for the total mobility by combining Equations (3.34), (3.36) and (3.37), and assuming no interaction between the two components (i.e. $\xi_{1}=\xi_{2}=\xi$ ).

$$
M=\frac{\nu c(1-c)}{\xi}
$$

\subsection{The Ludwig-Soret Effect}

In this section, the phenomenon of the Ludwig-Soret effect or thermal diffusion, which arises in a mixture if both the concentrations, and the temperature are non-uniform over the system, will be studied. For the derivation of the equation of the thermal diffusion, consider the multi-component isotropic fluids in which viscous phenomena may be neglected, and no external forces are present. Under these conditions, the pressure is uniform over the system if it is assumed that mechanical equilibrium is rapidly established. The entropy 
production can be expressed as [30]:

$$
\sigma=-J_{q}^{\prime} \cdot \frac{\nabla T}{T^{2}}-\sum_{l=1}^{n-1} J_{l} \cdot \frac{\left\{\nabla\left(\mu_{l}-\mu_{n}\right)\right\}_{T, p}}{T}
$$

where, $\sigma$ represents the entropy production in the polymer solution; $J_{q}^{\prime}$ is a coefficient related to heat flow; $J_{l}$ represents a coefficient related to diffusivity of $l^{\text {th }}$ component, $(l=1,2,3, \ldots,(n-1))$. With the help of Gibbs-Duhem equation

$$
\sum_{l=1}^{n} c_{l} \cdot \delta \mu_{l}=0 \quad(T \text { and } p \text { constant })
$$

Using Equation (3.40) to eliminate $\mu_{n}$ from Equation (3.39), it gives

$$
\begin{gathered}
\sigma=-J_{q}^{\prime} \cdot \frac{\nabla T}{T^{2}}-\sum_{l, m=1}^{n-1} A_{l m} \cdot \frac{\left(\nabla \mu_{m}\right)_{T, p}}{T} \\
\text { where } A_{l m}=\delta_{l m}+\frac{c_{m}}{c_{n}} \quad(l, m=1,2,3, \ldots n-1)
\end{gathered}
$$

The gradients of the chemical potential can also be expressed in terms of the concentration gradients as expressed below:

$$
\left(\nabla \mu_{m}\right)_{T, p}=\sum_{i=1}^{n-1} \mu_{m i}^{c} \nabla c_{i} \quad(m=1,2,3, \ldots, n-1)
$$

where, the matrix elements $\mu_{m i}^{c}$ are abbreviation for the derivatives $\left(\partial \mu_{m} / \partial c_{i}\right)_{T, p, c_{j}}(j \neq i)$.

Using Equations (3.42) and (3.43), the phenomenological equations for the fluxes and the thermodynamic forces, which appear in Equation (3.41), are now

$$
J_{q}^{\prime}=-L_{q q} \cdot \frac{\nabla T}{T^{2}}-\sum_{l, m, j=1}^{n-1} L_{q l} \cdot A_{l m} \cdot \frac{\left(\mu_{m j}^{c} \nabla c_{j}\right)}{T}
$$




$$
J_{i}=-L_{i q} \cdot \frac{\nabla T}{T^{2}}-\sum_{l, m, j=1}^{n-1} L_{i l} \cdot A_{l m} \cdot \frac{\left(\mu_{m j}^{c} \nabla c_{j}\right)}{T} \quad(i=1,2,3, \ldots n-1)
$$

with Onsager's relations,

$$
L_{i q}=L_{q i} \quad \& \quad L_{i l}=L_{l i} \quad(i, l=1,2,3, \ldots, n-1)
$$

The coefficients, $L_{q q}$ in Equation (3.44) and $L_{i l}$ in Equation (3.45), are related to the heat conductivity and diffusivity of the components of the mixture respectively. The coefficients $L_{i q}$ in Equation (3.45) are characteristics for the phenomenon of thermal diffusion, i.e. a flow of matter (flux) caused by the temperature gradient. It is usually called Ludwig-Soret effect in liquids. A reciprocal phenomena, viz. a heat flow caused by the concentration gradient also exists, as it is clear from Equation (3.44). This effect is called the Dufour effect and its magnitude depends on the coefficient $L_{q l}$.

In a closed reservoir with applied temperature gradient, the concentration gradient will build up until, in a final stationary state, the diffusion flow Equation (3.45) vanishes. The concentration gradients are given by:

$$
\begin{gathered}
0=-L_{i q} \cdot \frac{\nabla T}{T^{2}}-\sum_{l, m, j=1}^{n-1} L_{i l} \cdot A_{l m} \cdot \frac{\left(\mu_{m j}^{c} \nabla c_{j}\right)_{T, p}}{T} \quad(i=1,2,3, \ldots n-1) \\
\sum_{l, m, j=1}^{n-1} L_{i l} \cdot A_{l m} \cdot \frac{\left(\mu_{m j}^{c} \nabla c_{j}\right)_{T, p}}{T}=-L_{i q} \cdot \frac{\nabla T}{T^{2}} \quad(i=1,2,3, \ldots n-1) \\
\frac{\nabla c_{j}}{\nabla T}=-\frac{1}{T} \sum_{m, l, i=1}^{n-1} A_{m l}^{-1} \cdot\left(\mu^{c}\right)_{j m}^{-1} \cdot L_{l i}^{-1} \cdot L_{i q} \quad(j=1,2,3, \ldots, n-1)
\end{gathered}
$$

where, the reciprocal matrix of Equation (3.42) is given by [30]

$$
A_{m l}^{-1}=\delta_{m l}-c_{l} \quad(m, l=1,2,3, \ldots, n-1)
$$


Let us now consider, in somewhat more detail, the case of a binary solution $(n=2)$. Then it follows from Equation (3.42) that

$$
A_{11}=\frac{1}{c_{2}}
$$

and the phenomenological Equations (3.44) and (3.45) become:

$$
\begin{aligned}
& J_{q}^{\prime}=-L_{q q} \cdot \frac{\nabla T}{T^{2}}-L_{q 1} \cdot \frac{\mu_{11}^{c}}{c_{2} T} \cdot \nabla c_{1} \\
& J_{1}=-L_{1 q} \cdot \frac{\nabla T}{T^{2}}-L_{11} \cdot \frac{\mu_{11}^{c}}{c_{2} T} \cdot \nabla c_{1}
\end{aligned}
$$

with the Onsager's relation

$$
L_{1 q}=L_{q 1}
$$

and the inequalities which follows from the fact that the entropy source strength is positive definite [30]

$$
L_{q q} \geq 0 \quad \& \quad L_{11} \geq 0
$$

Instead of the phenomenological coefficients occurring in Equations (3.52) and (3.53), the following set of coefficients can be introduced:

$$
\begin{gathered}
\varphi=\frac{L_{q q}}{T^{2}} \quad \text { (heat conductivity) } \\
D^{\prime \prime}=\frac{L_{q 1}}{c_{2} T^{2}} \quad \text { (Dufour coefficient) } \\
D^{\prime}=\frac{L_{1 q}}{c_{1} c_{2} T^{2}} \quad \text { (thermal diffusion coefficient) } \\
D=\frac{L_{11} \mu_{11}^{c}}{c_{2} T} \quad \text { (ordinary diffusion coefficient) }
\end{gathered}
$$

The cross effect phenomena of thermal diffusion and Dufour effect are now given by the coefficients $D^{\prime}$ and $D^{\prime \prime}$ respectively. With the use of the above mentioned definitions from 
Equations (3.56) to (3.59), Equations 3.52 and 3.53 can take the following forms:

$$
\begin{aligned}
& J_{q}^{\prime}=-\varphi \cdot \nabla T-D^{\prime \prime} T \mu_{11}^{c} \cdot \nabla c_{1} \\
& J_{1}=-D^{\prime} c_{1} c_{2} \cdot \nabla T-D \cdot \nabla c_{1}
\end{aligned}
$$

Thus, Equations (3.60) and (3.61) are representative of the cross phenomena of Dufour effect (heat flow or flux caused by the concentration gradient) and thermal diffusion or Ludwig-Soret effect (mass flow or flux caused by the temperature gradient) respectively. It can be observed from Equation (3.61) that it consists of two parts, namely thermal diffusion part $\left(D^{\prime} c_{1} c_{2} \cdot \nabla T\right)$ and ordinary diffusion part $\left(D \cdot \nabla c_{1}\right)$. Now, the ordinary diffusion term can also be written in terms of the chemical potential difference and mobility [78]. Equation 3.61 takes the following form:

$$
J_{1}=-D^{\prime} c_{1} c_{2} \cdot \nabla T-M \cdot \nabla\left(\mu_{2}-\mu_{1}\right) \quad \text { (using equation (3.42)) }
$$

where, mobility $M=L_{11} /\left(c_{2} T\right), D^{\prime}$ is thermal diffusion coefficient, $c_{1}$ and $c_{2}$ are the respective concentration of component 1 and 2 , and $\mu_{1}$ and $\mu_{2}$ are the respective chemical potentials of the component 1 and 2 in the binary solution. 


\section{CHAPTER 4}

\section{GOVERNING EQUATIONS AND}

\section{METHOD OF SOLUTIONS}

This chapter describes the model development for the TIPS method of phase separation involving the thermal diffusion phenomena under the externally imposed spatial temperature gradient in the binary polymer solution. Figure 4.1 shows a flowchart of mathematical modelling and computational simulation procedures utilized in order to accomplish the objectives of the research thesis. As per Section 2.3, the first and the foremost objective of this thesis is to develop the mathematical model based on the available theories, such as the non-linear Cahn-Hilliard theory, Flory-Huggins theory and Ludwig-Soret theory, which altogether best describe the TIPS method of the phase separation in a binary polymer solution as well as the thermal diffusion phenomenon under an externally imposed spatial linear temperature gradient. The temperature gradient, which is assumed to be linear along the axis (i.e. $x$ or $y$-axis) in the polymer solution, will be defined later in this chapter. Also mobility, $M$ which is assumed to be function of concentration of solution, will be defined. The model is then solved using the appropriate computational methods (i.e. numerical methods) and computer programming language. Finally, the numerical results obtained from the computer simulation of the mathematical model are analyzed and presented in such a manner that they can be validated with the published experimental and/or numerical results if any. 


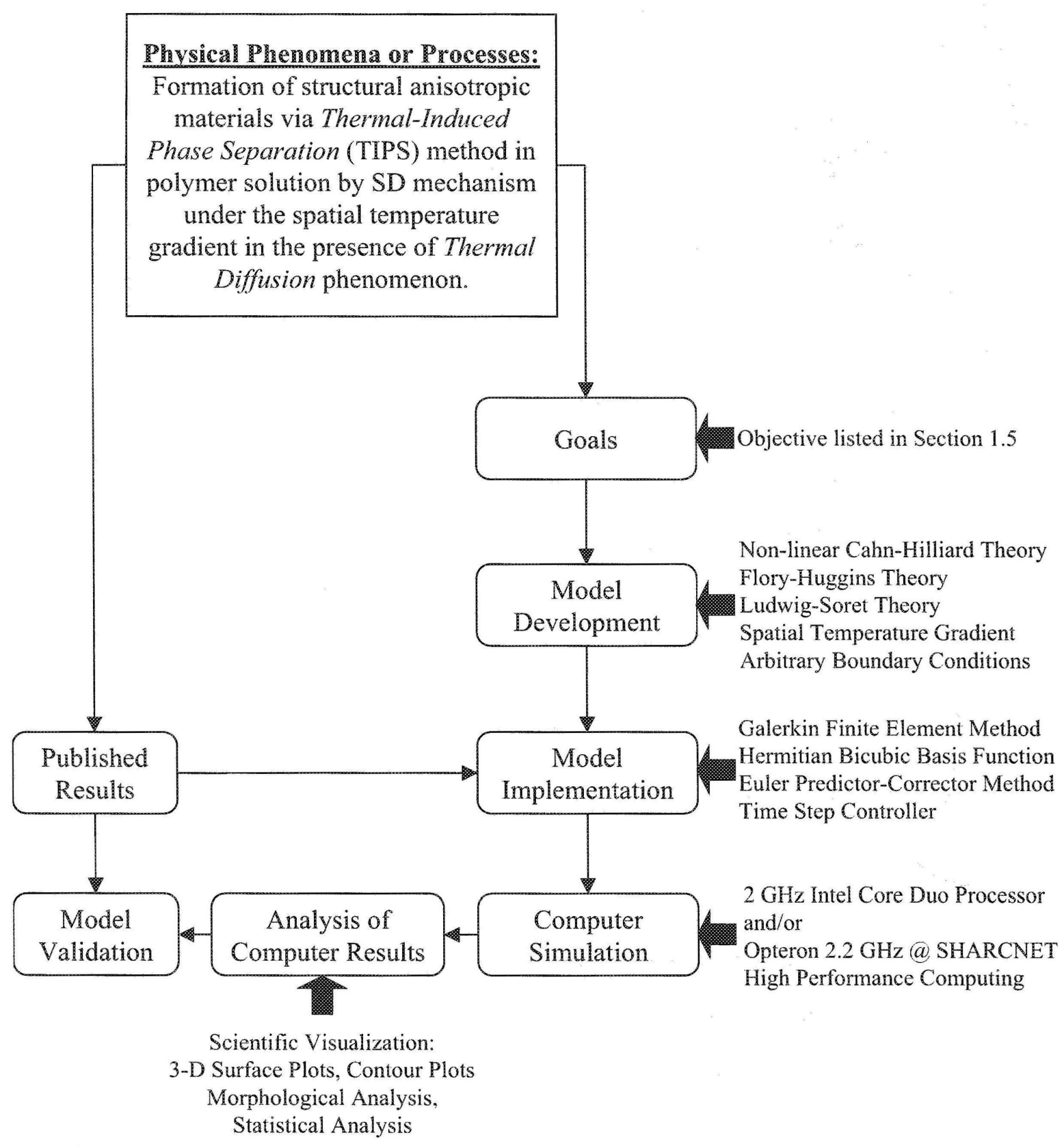

Figure 4.1: A flowchart of mathematical modelling and computational simulation procedures for the accomplishment of the objectives of the research thesis. 
In this thesis, the following steps were followed for the computational modelling and simulation of the TIPS method of the phase separation in the polymer solution involving the thermal diffusion phenomenon.

1. Specify the independent and the dependent variables of the mathematical model. The independent variables include time and space, where as the dependent variable include the solvent concentration for the TIPS method involving the thermal diffusion phenomenon.

2. Derive the governing time-dependent partial differential equation to describe the TIPS method of phase separation via the SD mechanism in a binary polymer solution with an externally imposed spatial linear temperature gradient along with thermal diffusion phenomenon for the formation of the functional anisotropic materials taking into account above mentioned independent and dependent variables. The non-linear Cahn-Hilliard theory, the Flory-Huggins theory and Ludwig-Soret theory are used for derivation of the governing equation for the overall process of the phase separation in the binary polymer solution.

3. Derive the appropriate boundary conditions for the study of the process of the phase separation (TIPS) in polymer solution for the formation of the structural anisotropic polymeric materials. The boundary conditions used in the derivation of the governing equation are the zero mass flux and the natural boundary conditions.

4. Nondimensionalize the governing equation and boundary conditions obtained in steps 2 and 3 , that describe the formation of the structural anisotropic polymeric materials by the TIPS method of phase separation via the SD mechanism involving the thermal diffusion under the influence of an externally imposed spatial linear temperature gradient in binary polymer solutions and the dimensionless parameters obtained are listed. 
5. Develop the Galerkin finite element method formulations of the dimensionless governing equation and boundary conditions obtained in step 4 , that describe the formation of the structural anisotropic polymeric materials via the TIPS method of phase separation via the SD mechanism involving the thermal diffusion under the influence of an externally imposed spatial linear temperature gradient in binary polymer solutions. Galerkin finite element method formulations include the derivation of equations of the weighted residual vector and the jacobian matrix.

6. Implement and solve the Galerkin finite element method formulations obtained in step 5 by choosing the appropriate dimensionless parameter values and with the help of the computer programming language, such as FORTRAN 7\%. Investigate the influence of the parameters that affect the formation of the structural anisotropic polymeric materials via the TIPS method of phase separation via the SD mechanism involving the thermal diffusion under the influence of an externally imposed spatial linear temperature gradient in binary polymer solutions.

7. Analyze the computational simulation output obtained in step 6 using the scientific visualization methods, i.e. 3-D surface plots and contour plots and the morphological analysis.

8. Validate the simulation results using the published experimental and/or computational (numerical) results if any.

\subsection{Generalized Governing Equation}

The continuity equation for mass flow is expressed as:

$$
\frac{\partial c}{\partial t}=-\vec{\nabla} \cdot \overrightarrow{\mathfrak{J}}
$$


where $c$ is the concentration of solvent in terms of the volume fraction, and $\mathbb{J}$ is the mass flux caused by combined phenomena of non-fickian diffusion (uphill-diffusion) and thermal diffusion under an externally imposed spatial temperature gradient in a binary polymer solution. Under this conditions, the the mass flux, $\mathbb{J}$ is expressed as below:

$$
\mathbb{J}=-M(c) \cdot \nabla\left(\mu_{2}-\mu_{1}\right)-D^{\prime} c(1-c) \cdot \nabla T \quad \text { (from Equation (3.62)) }
$$

where, $M(c)$ is the concentration dependent mobility, $\mu_{1}$ and $\mu_{2}$ are the respective chemical potentials of the component 1 (solvent) and component 2 (polymer) in the solution, and $D^{\prime}$ is the coefficient of thermal diffusion. Above Equation (4.2) is composed of of two parts: part one represents the TIPS method of phase separation by SD mechanism, and part two represents the Ludwig-Soret effect or thermal diffusion phenomenon in the solution under the influence of the temperature gradient.

Substituting the value of J from Equation (4.2) in Equation (4.1),

$$
\frac{\partial c}{\partial t}=\nabla \cdot\left[M(c) \cdot \nabla\left(\mu_{2}-\mu_{1}\right)+D^{\prime} c(1-c) \cdot \nabla T\right]
$$

And also, substituting the value of $\left(\mu_{2}-\mu_{1}\right)$ from Equation (3.20) in Equation (4.3), it becomes

$$
\frac{\partial c}{\partial t}=\nabla \cdot\left[M(c) \cdot \nabla\left(\frac{\partial f(c)}{\partial c}-2 \kappa \nabla^{2} c\right)\right]+\nabla \cdot\left[D^{\prime} c(1-c) \cdot \nabla T\right]
$$

where, $f(c)$ represents the Flory-Huggins $(\mathrm{F}-\mathrm{H})$ free energy, and $\kappa$ is gradient energy coefficient for a polymer solution. In Equation (4.4), the first term, on right hand side, represents the non-linear Cahn-Hilliard theory describing the phase separation in a polymer solution by the spinodal decomposition (SD) mechanism, and the second term represents the phenomenon of thermal diffusion arising due to an externally imposed spatial temperature 
gradient across a polymer solution sample. Thus, Equation (4.4) presents the overall process of thermal-induced phase separation (TIPS) by the SD mechanism involving thermal diffusion phenomenon in a binary polymer solution under the temperature gradient, and therefore, it forms the important base of this research work.

The Flory-Huggins $(\mathrm{F}-\mathrm{H})$ free energy, $f(c)$ term in Equation (4.4) can be given as:

$$
f(c)=\frac{k_{B} T}{\nu}\left[\frac{c}{N_{1}} \ln c+\frac{(1-c)}{N_{2}} \ln (1-c)+\chi c(1-c)\right] \quad \text { (see Equation (3.5)) }
$$

where, $k_{B}$ is Boltzmann's constant, $T$ is a temperature, $\nu$ is a cell volume, $N_{1}$ and $N_{2}$ are the respective degree of polymerization of component 1 and 2 , and $\chi$ is Flory's interaction parameter.

The Flory's interaction parameter, $\chi$ is known to be the function of temperature and is expressed as the following (refer to Subsection 3.1.2):

$$
\chi=\frac{1}{2}-\psi\left(1-\frac{\theta}{T}\right)
$$

where, $\psi$ is the dimensionless entropy of dilution parameter, and $\theta$ is the theta temperature

\subsubsection{Spatial Temperature Gradient}

In this research work, a linear spatial temperature gradient, similar to what Lee et al [22] adopted in their work, has been assumed to exist along a binary polymer solution sample. Following is the expression for an externally imposed spatial linear temperature gradient across a binary polymer solution samples, which is assumed to exist along the $x$-direction 
in the sample.

$$
T(x)=\left(\frac{T_{2}-T_{1}}{x_{2}-x_{1}}\right)\left(x-x_{1}\right)+T_{1}, \quad \text { for } \quad T_{1} \leq T_{2} \quad \text { and } \quad x_{1} \leq x_{2}
$$

where, $T(x)$ is the spatial temperature, and $T_{1}$ and $T_{2}$ are the lower and upper limits of the linear temperature gradient corresponding to the positions $x_{1}$ and $x_{2}$ on the $x$-axis.

For the one-dimensional study, the linear temperature gradient is assumed to exist along the study domain of length $L$ during the phase separation phenomena, whereas for the two-dimensional study, the linear spatial temperature gradient is assumed to exist only along the $x$-direction of a square computational domain with the dimension $L \times L$ (i.e. $0 \leq x \leq L$ and $0 \leq y \leq L)$. It implies that there is no temperature gradient along the $y$-axis in the two dimensional study (i.e. $\partial T(y) / \partial y=0$ ).

\subsubsection{Concentration Dependent Mobility}

Unlike the conventional numerical studies of the Cahn-Hilliard (C-H) equation, the mobility, $M$ has been assumed to be the function of concentration of the components in the solution (refer to Section (3.4)). Either the slow mode theory or the fast mode theory can be employed to calculate the total mobility of polymer solution. The slow mode theory has been used in this research work, as it better represents the slow diffusion behavior exhibited by a polymer solution. According the slow mode theory and in absence of the entanglement effects, the total mobility can be expressed as:

$$
M=\frac{\nu c(1-c)}{\xi}
$$

where, $\nu$ is volume of the segment, and $\xi$ is the frictional coefficient per segment of constituent components in the solution. 
Now, substituting the value of $f(c)$ from Equation (4.5) along with its derivatives in Equation (4.4), and then on simplification, Equation (4.4) reduces to

$$
\begin{aligned}
\frac{\partial c}{\partial t} & =\frac{k_{B} \cdot \nabla M(c)}{\nu}\left[\nabla T\left(\frac{1}{N_{1}}-\frac{1}{N_{2}}+\frac{\ln c}{N_{1}}-\frac{\ln (1-c)}{N_{2}}+\chi(1-2 c)\right)+(1-2 c) T \nabla T \frac{\partial \chi}{\partial T}\right. \\
& \left.+\nabla c \cdot T\left(\frac{1}{N_{1} c}+\frac{1}{N_{2}(1-c)}-2 \chi\right)\right]+\frac{k_{B} \cdot M(c)}{\nu}\left[T(\nabla c)^{2}\left(\frac{-1}{N_{1} c^{2}}+\frac{1}{N_{2}(1-c)^{2}}\right)\right. \\
& +\nabla^{2} T\left(\frac{1}{N_{1}}-\frac{1}{N_{2}}+\frac{\ln c}{N_{1}}-\frac{\ln (1-c)}{N_{2}}+\chi(1-2 c)\right)-4 \nabla c \cdot T \cdot \nabla T \cdot \frac{\partial \chi}{\partial T} \\
& +\left(T \cdot \nabla^{2} c+2 \nabla c \cdot \nabla T\right)\left(\frac{1}{N_{1} c}+\frac{1}{N_{2}(1-c)}-2 \chi\right)+2(1-2 c) \cdot(\nabla T)^{2} \cdot \frac{\partial \chi}{\partial T} \\
& \left.+(1-2 c) \cdot T \cdot\left(\nabla T \cdot \nabla T+T \cdot \nabla^{2} T\right) \cdot \frac{\partial^{2} \chi}{\partial T^{2}}\right]-2 \kappa \cdot \nabla M(c) \cdot \nabla^{3} c-2 \kappa \cdot M(c) \cdot \nabla^{4} c \\
& +D^{\prime} \cdot(1-2 c) \cdot \nabla c \cdot \nabla T+D^{\prime} \cdot c \cdot(1-c) \cdot \nabla^{2} T
\end{aligned}
$$

Substituting Equations (4.6), (4.7), (4.8) and their derivatives in Equation (4.9), and then further simplifying it, we get

$$
\begin{aligned}
\frac{\partial c}{\partial t} & =\frac{k_{B}}{\xi}\left[\left(\frac{3-4 c+(1-2 c) \ln c}{N_{1}}\right)-\left(\frac{1-4 c+(1-2 c) \ln (1-c)}{N_{2}}\right)+4\left(c-c^{2}\right)(2 \psi-1)\right. \\
& \left.-\psi+\frac{1}{2}\right] \nabla c \cdot \nabla T+\frac{k_{B} T}{\xi}\left[\frac{-1}{N_{1}}+\frac{1}{N_{2}}-2 \chi(1-2 c)\right](\nabla c)^{2}-\left(\frac{2 \kappa \nu}{\xi}\right)(1-2 c) \nabla c \cdot \nabla^{3} c \\
& +\frac{k_{B} T}{\xi}\left[\frac{(1-c)}{N_{1}}+\frac{c}{N_{2}}-2 \chi\left(c-c^{2}\right)\right] \nabla^{2} c-\left(\frac{2 \kappa \nu}{\xi}\right)\left(c-c^{2}\right) \nabla^{4} c \\
& +D^{\prime}(1-2 c) \nabla c \cdot \nabla T
\end{aligned}
$$

Above Equation (4.10) is the generalized governing equation for the TIPS method of phase separation by the SD mechanism in a binary polymer solution involving the thermal diffusion phenomenon under an externally imposed spatial linear temperature gradient.

Based on Equation (4.10), the governing equation for the one-dimensional study can be 
expressed as:

$$
\begin{aligned}
\frac{\partial c}{\partial t} & =\frac{k_{B}}{\xi}\left[\left(\frac{3-4 c+(1-2 c) \ln c}{N_{1}}\right)-\left(\frac{1-4 c+(1-2 c) \ln (1-c)}{N_{2}}\right)+4\left(c-c^{2}\right)(2 \psi-1)\right. \\
& \left.-\psi+\frac{1}{2}\right]\left(\frac{\partial c}{\partial x}\right)\left(\frac{T_{2}-T_{1}}{x_{2}-x_{1}}\right)+\frac{k_{B} T}{\xi}\left[\frac{-1}{N_{1}}+\frac{1}{N_{2}}-2 \chi(1-2 c)\right]\left(\frac{\partial c}{\partial x}\right)\left(\frac{\partial c}{\partial x}\right) \\
& +\frac{k_{B} T}{\xi}\left[\frac{(1-c)}{N_{1}}+\frac{c}{N_{2}}-2 \chi\left(c-c^{2}\right)\right]\left(\frac{\partial^{2} c}{\partial x^{2}}\right)-\left(\frac{2 \nu \kappa}{\xi}\right)(1-2 c)\left(\frac{\partial c}{\partial x}\right)\left(\frac{\partial^{3} c}{\partial x^{3}}\right) \\
& -\left(\frac{2 \nu \kappa}{\xi}\right)\left(c-c^{2}\right)\left(\frac{\partial^{4} c}{\partial x^{4}}\right)+D^{\prime}(1-2 c)\left(\frac{\partial c}{\partial x}\right)\left(\frac{T_{2}-T_{1}}{x_{2}-x_{1}}\right)
\end{aligned}
$$

and similarly, the governing equation for the two dimensional study can be written as:

$$
\begin{aligned}
\frac{\partial c}{\partial t} & =\frac{k_{B}}{\xi}\left[\left(\frac{3-4 c+(1-2 c) \ln c}{N_{1}}\right)-\left(\frac{1-4 c+(1-2 c) \ln (1-c)}{N_{2}}\right)+4\left(c-c^{2}\right)(2 \psi-1)\right. \\
& \left.-\psi+\frac{1}{2}\right]\left(\frac{\partial c}{\partial x}\right)\left(\frac{T_{2}-T_{1}}{x_{2}-x_{1}}\right)+\frac{k_{B} T}{\xi}\left[\frac{-1}{N_{1}}+\frac{1}{N_{2}}-2 \chi(1-2 c)\right]\left(\frac{\partial c}{\partial x} \cdot \frac{\partial c}{\partial x}+\frac{\partial c}{\partial y} \cdot \frac{\partial c}{\partial y}\right) \\
& +\frac{k_{B} T}{\xi}\left[\frac{(1-c)}{N_{1}}+\frac{c}{N_{2}}-2 \chi\left(c-c^{2}\right)\right]\left(\frac{\partial^{2} c}{\partial x^{2}}+\frac{\partial^{2} c}{\partial y^{2}}\right)-\left(\frac{2 \kappa \nu}{\xi}\right)(1-2 c)\left(\frac{\partial c}{\partial x} \cdot \frac{\partial^{3} c}{\partial x^{3}}\right. \\
& \left.+\frac{\partial c}{\partial x} \cdot \frac{\partial^{3} c}{\partial x \partial y^{2}}+\frac{\partial c}{\partial y} \cdot \frac{\partial^{3} c}{\partial x^{2} \partial y}+\frac{\partial c}{\partial y} \cdot \frac{\partial^{3} c}{\partial y^{3}}\right)-\left(\frac{2 \kappa \nu}{\xi}\right)\left(c-c^{2}\right)\left(\frac{\partial^{4} c}{\partial x^{4}}+2 \frac{\partial^{4} c}{\partial x^{2} \partial y^{2}}+\frac{\partial^{4} c}{\partial y^{4}}\right) \\
& +D^{\prime}(1-2 c)\left(\frac{\partial c}{\partial x}\right)\left(\frac{T_{2}-T_{1}}{x_{2}-x_{1}}\right)
\end{aligned}
$$

The governing Equations (4.10), (4.11) and (4.12) are then non-dimensionalized using the scaling relationships presented in Appendix A. The parameters with superscripted asterisk represent the dimensionless variables. Therefore, the dimensionless form of the generalized governing Equation (4.10) is

$$
\begin{aligned}
\frac{\partial c^{*}}{\partial t^{*}} & =D^{*}\left[\left(\frac{3-4 c^{*}+\left(1-2 c^{*}\right) \ln c^{*}}{N_{1}}\right)-\left(\frac{1-4 c^{*}+\left(1-2 c^{*}\right) \ln \left(1-c^{*}\right)}{N_{2}}\right)+4\left(c^{*}-c^{*^{2}}\right)\right. \\
& \left.\cdot(2 \psi-1)-\psi+\frac{1}{2}\right] \nabla^{*} c^{*} \cdot \nabla^{*} T^{*}+D^{*} T^{*}\left[\frac{-1}{N_{1}}+\frac{1}{N_{2}}-2 \chi\left(1-2 c^{*}\right)\right]\left(\nabla^{*} c^{*}\right)^{2} \\
& -\left(1-2 c^{*}\right) \nabla^{*} c^{*} \cdot \nabla^{*^{3}} c^{*}+D^{*} T^{*}\left[\frac{\left(1-c^{*}\right)}{N_{1}}+\frac{c^{*}}{N_{2}}-2 \chi\left(c^{*}-c^{*^{2}}\right)\right] \nabla^{*^{2}} c^{*} \\
& -\left(c^{*}-c^{*^{2}}\right) \nabla^{*^{4}} c^{*}+D^{\prime *}\left(1-2 c^{*}\right) \nabla^{*} c^{*} \cdot \nabla^{*} T^{*}
\end{aligned}
$$


the dimensionless form of the one-dimensional governing Equation (4.11) is given as:

$$
\begin{aligned}
\frac{\partial c^{*}}{\partial t^{*}}= & D^{*}\left[\left(\frac{3-4 c^{*}+\left(1-2 c^{*}\right) \ln c^{*}}{N_{1}}\right)-\left(\frac{1-4 c^{*}+\left(1-2 c^{*}\right) \ln \left(1-c^{*}\right)}{N_{2}}\right)+4\left(c^{*}-c^{*^{2}}\right)\right. \\
\cdot & \left.(2 \psi-1)-\psi+\frac{1}{2}\right]\left(\frac{\partial c^{*}}{\partial x^{*}}\right)\left(\frac{T_{2}^{*}-T_{1}^{*}}{x_{2}^{*}-x_{1}^{*}}\right)+D^{*} T^{*}\left[\frac{-1}{N_{1}}+\frac{1}{N_{2}}-2 \chi\left(1-2 c^{*}\right)\right]\left(\frac{\partial c^{*}}{\partial x^{*}}\right) \\
\cdot & \left(\frac{\partial c^{*}}{\partial x^{*}}\right)+D^{*} T^{*}\left[\frac{\left(1-c^{*}\right)}{N_{1}}+\frac{c^{*}}{N_{2}}-2 \chi\left(c^{*}-c^{*^{2}}\right)\right]\left(\frac{\partial^{2} c^{*}}{\partial x^{2}}\right)-\left(1-2 c^{*}\right)\left(\frac{\partial c^{*}}{\partial x^{*}}\right) \\
\therefore & \left(\frac{\partial^{3} c^{*}}{\partial x^{* 3}}\right)-\left(c^{*}-c^{*^{2}}\right)\left(\frac{\partial^{4} c^{*}}{\partial x^{*}}\right)+D^{\prime *}\left(1-2 c^{*}\right)\left(\frac{\partial c^{*}}{\partial x^{*}}\right)\left(\frac{T_{2}^{*}-T_{1}^{*}}{x_{2}^{*}-x_{1}^{*}}\right)
\end{aligned}
$$

and similarly, dimensionless form of the two-dimensional governing Equation (4.12) is written as shown below:

$$
\begin{aligned}
\frac{\partial c^{*}}{\partial t^{*}}= & D^{*}\left[\left(\frac{3-4 c^{*}+\left(1-2 c^{*}\right) \ln c^{*}}{N_{1}}\right)-\left(\frac{1-4 c^{*}+\left(1-2 c^{*}\right) \ln \left(1-c^{*}\right)}{N_{2}}\right)+4\left(c^{*}-c^{*^{2}}\right)\right. \\
& \left.\cdot(2 \psi-1)-\psi+\frac{1}{2}\right]\left(\frac{\partial c^{*}}{\partial x^{*}}\right)\left(\frac{T_{2}^{*}-T_{1}^{*}}{x_{2}^{*}-x_{1}^{*}}\right)+D^{*} T^{*}\left[\frac{-1}{N_{1}}+\frac{1}{N_{2}}-2 \chi\left(1-2 c^{*}\right)\right] \\
& \cdot\left(\frac{\partial c^{*}}{\partial x^{*}} \cdot \frac{\partial c^{*}}{\partial x^{*}}+\frac{\partial c^{*}}{\partial y} \cdot \frac{\partial c^{*}}{\partial y^{*}}\right)+D^{*} T^{*}\left[\frac{\left(1-c^{*}\right)}{N_{1}}+\frac{c^{*}}{N_{2}}-2 \chi\left(c^{*}-c^{*^{2}}\right)\right]\left(\frac{\partial^{2} c^{*}}{\partial x^{*^{2}}}+\frac{\partial^{2} c^{*}}{\partial y^{*^{2}}}\right) \\
- & \left(1-2 c^{*}\right)\left(\frac{\partial c^{*}}{\partial x^{*}} \cdot \frac{\partial^{3} c^{*}}{\partial x^{*^{3}}}+\frac{\partial c^{*}}{\partial x^{*}} \cdot \frac{\partial^{3} c^{*}}{\partial x^{*} \partial y^{*^{2}}}+\frac{\partial c^{*}}{\partial y^{*}} \cdot \frac{\partial^{3} c^{*}}{\partial x^{*^{2}} \partial y^{*}}+\frac{\partial c^{*}}{\partial y^{*}} \cdot \frac{\partial^{3} c^{*}}{\partial y^{* 3}}\right) \\
- & \left(c^{*}-c^{*^{2}}\right)\left(\frac{\partial^{4} c^{*}}{\partial x^{* *}}+2 \frac{\partial^{4} c^{*}}{\partial x^{*} \partial y^{*^{2}}}+\frac{\partial^{4} c^{*}}{\partial y^{* 4}}\right)+D^{\prime *}\left(1-2 c^{*}\right)\left(\frac{\partial c^{*}}{\partial x^{*}}\right)\left(\frac{T_{2}^{*}-T_{1}^{*}}{x_{2}^{*}-x_{1}^{*}}\right)
\end{aligned}
$$

The dimensionless governing Equations (4.14) and (4.15) are then solved numerically using the Galerkin finite element method, which is discussed later in the chapter.

\subsection{Auxiliary Equations}

As mentioned earlier, the infinitesimal concentration fluctuations are always present in the polymer solution initially even in the single-phase region. These infinitesimal concentration fluctuations are sufficient to drive the process of phase separation by the spinodal decompo-

sition (SD) mechanism. Therefore, the expression of the dimensionless initial concentration 
can be given as:

$$
c^{*}\left(t^{*}=0\right)=c_{0}^{*}+\delta c^{*}\left(t^{*}=0\right)
$$

where, $c_{0}^{*}$ is the dimensionless initial concentration, and $\delta c^{*}\left(t^{*}=0\right)$ represents any deviation from the average initial concentration $c_{0}^{*}$ or the infinitesimally small concentration fluctuations, which may be present in the solution. Chan and Rey [28] used the Monte Carlo simulations incorporated with the linearization approximation and the equipartition theorem in their work for the development of the expression for the initial condition for the phase separation in polymer solution.

\subsubsection{Boundary Conditions}

In order to solve Equations (4.14) and (4.15) numerically, two kinds of boundary conditions are taken into consideration: the zero mass flux boundary conditions and the natural boundary condition.

The zero mass flux boundary condition refers to the system in which it is assumed that there is no exchange of mass with its surrounding. It is obtained by setting Equation (3.21) equal to zero [84]. For one-dimensional study, the dimensionless form of the zero mass flux boundary condition can be expressed as follows [28]:

$$
\frac{\partial^{3} c^{*}}{\partial x^{*^{3}}}=0 \quad \text { for } \quad t^{*}>0, \text { and } \quad x^{*}=0 \quad \& \quad x^{*}=1
$$

and similarly, the dimensionless form of the zero mass flux boundary conditions for the two-dimensional study is given as:

$$
\frac{\partial^{3} c^{*}}{\partial x^{*^{3}}}+\frac{\partial^{3} c^{*}}{\partial x^{*} \partial y^{*^{2}}}=0 \quad \text { for } \quad t^{*}>0, \quad \text { and } \quad x^{*}=0 \quad \& \quad x^{*}=1
$$




$$
\frac{\partial^{3} c^{*}}{\partial y^{*^{3}}}+\frac{\partial^{3} c^{*}}{\partial x^{*^{2}} \partial y^{*}}=0 \quad \text { for } \quad t^{*}>0, \quad \text { and } \quad y^{*}=0 \quad \& \quad y^{*}=1
$$

The natural boundary condition is obtained from the variational analysis, and is expressed in generalized form as [85]:

$$
(\nabla c) \cdot \mathbf{n}=0
$$

where, $\mathbf{n}$ is the outward unit normal to a bounding surface. The dimensionless natural boundary condition for the onc-dimensional study is given as:

$$
\frac{\partial c^{*}}{\partial x^{*}}=0 \quad \text { for } \quad t^{*}>0, \text { and } \quad x^{*}=0 \quad \& \quad x^{*}=1
$$

In addition to Equation (4.21), the dimensionless natural boundary condition for two-dimensional study in $y$-direction is

$$
\frac{\partial c^{*}}{\partial y^{*}}=0 \quad \text { for } \quad t^{*}>0, \quad \text { and } \quad y^{*}=0 \quad \& \quad y^{*}=1
$$

\subsection{Method of Solution}

As the governing equation derived in the study is a non-linear fourth order partial differential equation, the numerical method of finite element (Galerkin finite element method to be specific) is employed to solve it. In the finite element method, the domain is divided into subdomains commonly referred to as finite elements [86]. These subdomains or elements are called the fundamental building block of the finite element method. The Galerkin finite element method for the two-dimensional problem is described briefly in the following subsection, and also described are the first order implicit Euler predictor-corrector method (for time integration) and an adaptive time-step control mechanism to reduce the 
computational time while maintaining accuracy of the numerical solution.

\subsubsection{Galerkin Finite Element Method}

Consider a general non-linear partial differential equation with appropriate initial and boundary condition as shown below:

$$
L u(\cdot)-f=0
$$

where, $L$ is a co-efficient of function $u(\cdot)$, and $f$ is an arbitrary constant. Let $u_{a}$ be the approximate solution of the above mentioned partial differential Equation (4.23) and can given by following expression:

$$
u_{a}(\cdot)=\sum_{j=1}^{N} u_{j} \cdot \phi_{j}(\cdot)
$$

where, $u_{j}$ represents the set of unknown coefficients; $\phi_{j}$ represents the set of linearly independent functions, which are chosen to be polynomials that satisfy certain boundary conditions imposed on the given equation; and $N$ is the number of nodes in the spatial discretization of the computational domain. Substitution of $u_{a}$ from Equation (4.24) into Equation (4.23) results in the residual $R(\cdot)$ as given below.

$$
L u_{a}(\cdot)-f=R(\cdot) \neq 0
$$

Here, the objective is to find the set of undetermined coefficients $u_{j}$ such that the residual is minimized in some sense. A straightforward scheme would be to set the double integral of $R(\cdot)$ to zero for two- dimensional case to yield the residual vector $F_{i}$.

$$
F_{i}=\iint_{A} R(\cdot) \cdot w_{i}(\cdot) d A=\iint_{A} R(\cdot) \cdot \phi_{i}(\cdot) d A=0 \quad(\text { where } i=1,2,3, \ldots, N)
$$


where, $w_{i}$ s represent the weighting functions. For Galerkin finite element method, the weighting functions are chosen to be the basis functions, $\phi_{i}$ s (i.e. Hermitian bicubic basis function to be specific). Equation (4.26) yields a set of $N$ independent simultaneous equations, which can be solved simultaneously with the use of both Newton-Raphson iterative method and first-order implicit Euler predictor-corrector method.

The Hermitian bicubic basis functions are the appropriate choice for the basis functions for solution of the two-dimensional fourth-order partial differential equations such as the one represented by the governing Equation (4.13). The approximate solution in two dimensions is given by

$$
u_{a}(\cdot)=\sum_{j=1}^{N}\left(u_{j} \cdot \phi_{j, 0}+\frac{\partial u_{j}}{\partial x} \cdot \phi_{j, 1}+\frac{\partial u_{j}}{\partial y} \cdot \phi_{j, 2}+\frac{\partial u_{j}}{\partial x \partial y} \cdot \phi_{j, 3}\right)
$$

Equation (4.24) can also be expressed as shown below by consolidating the coefficients together as $U_{j}$ and by consolidating the Hermitian bicubic basis functions together as $\Phi_{j}$.

$$
u_{a}(\cdot)=\sum_{j=1}^{4 N} U_{j} \cdot \Phi_{j}
$$

where,

$$
\begin{gathered}
U_{j}=u_{k}, \quad \Phi_{j}=\phi_{k, 0} \quad \text { for }\left\{\begin{array}{l}
j=1,5,9,13, \ldots,(4 N-3) \\
k=1,2,3,4, \ldots, N
\end{array}\right\} \\
U_{j}=\frac{\partial u_{k}}{\partial x}, \quad \Phi_{j}=\phi_{k, 1} \\
\text { for } \quad\left\{\begin{array}{l}
j=2,6,10,14, \ldots,(4 N-2) \\
k=1,2,3,4, \ldots, N
\end{array}\right\} \\
U_{j}=\frac{\partial u_{k}}{\partial y}, \quad \Phi_{j}=\phi_{k, 2} \quad \text { for }\left\{\begin{array}{ll}
j=3,7,11,15, \ldots,(4 N-1) \\
k=1,2,3,4, \ldots, N
\end{array}\right\}
\end{gathered}
$$




$$
U_{j}=\frac{\partial^{2} u_{k}}{\partial x \partial y}, \quad \Phi_{j}=\phi_{k, 3} \quad \text { for } \quad\left\{\begin{array}{r}
j=4,8,12,16, \ldots, 4 N \\
k=1,2,3,4, \ldots, N
\end{array}\right\}
$$

The weighted residual of the dimensionless governing Equation (4.13) is expressed as follows:

$$
\begin{aligned}
F_{i} & =\int_{0}^{1} \int_{0}^{1}\left[\frac{\partial c^{*}}{\partial t^{*}}-D^{*}\left\{\left(\frac{3-4 c^{*}+\left(1-2 c^{*}\right) \ln c^{*}}{N_{1}}\right)-\left(\frac{1-4 c^{*}+\left(1-2 c^{*}\right) \ln \left(1-c^{*}\right)}{N_{2}}\right)\right.\right. \\
& \left.+4\left(c^{*}-c^{*^{2}}\right)(2 \psi-1)-\psi+\frac{1}{2}\right\} \nabla^{*} c^{*} \cdot \nabla^{*} T^{*}-D^{*} T^{*}\left\{\frac{-1}{N_{1}}+\frac{1}{N_{2}}-2 \chi\left(1-2 c^{*}\right)\right\} \\
& \cdot\left(\nabla^{*} c^{*}\right)^{2}+\left(1-2 c^{*}\right) \nabla^{*} c^{*} \cdot \nabla^{*^{3}} c^{*}+\left(c^{*}-c^{*^{2}}\right) \nabla^{*^{4}} c^{*}-D^{*}\left(1-2 c^{*}\right) \nabla^{*} c^{*} \cdot \nabla^{*} T^{*} \\
& \left.-D^{*} T^{*}\left\{\frac{\left(1-c^{*}\right)}{N_{1}}+\frac{c^{*}}{N_{2}}-2 \chi\left(c^{*}-c^{*^{2}}\right)\right\} \nabla^{*^{2}} c^{*}\right] \Phi_{i} d x^{*} d y^{*}, \quad(i=1,2,3, \ldots, N)(4.33)
\end{aligned}
$$

where,

$$
c^{*}=\sum_{j=1}^{4 N} C_{j} \cdot \Phi_{j}
$$

and $\nabla^{*}=\left(\partial / \partial x^{*}\right) \hat{i}+\left(\partial / \partial y^{*}\right) \hat{j}$ for two-dimensional study. Equation (4.34) is equivalent to Equation (4.28). The fourth-order partial derivative term in Equation (4.33) can be reduced to lower order by using the divergence theorem

$$
\int_{A} \alpha \vec{\nabla} \cdot \vec{\beta} d A=\int_{S} \alpha \mathbf{n} \cdot \vec{\beta} d S-\int_{A}(\vec{\nabla} \alpha) \cdot \vec{\beta} d A
$$

where, $\alpha$ is a scalar, $\beta$ is a vector, and $S$ is the boundary of the domain $A$.

\subsubsection{Euler's Predictor-Corrector Method}

This subsection briefly describes the first-order implicit Euler predictor-corrector method, which is employed for time integration of the governing equations. A fundamental source of error in Euler's method is that the derivative at the beginning of the interval is assumed to apply across the entire interval. One method to solve the estimate of the slope involves 
the determination of two derivatives for the interval, one at the initial point and one at the end point. The two derivatives are then averaged to obtain an improved estimate of the slope for the entire interval. This method is also called Heun's method, and is depicted graphically in Figure 4.1 in which Figure ?? describes the predictor method (also called Euler's forward method), and Figure ?? describes the corrector method (also called Euler's backward method).

Now, the slope at the beginning of an interval $y_{i}^{\prime}=y_{i}+f\left(x_{i}, y_{i}\right)$ is used to extrapolate

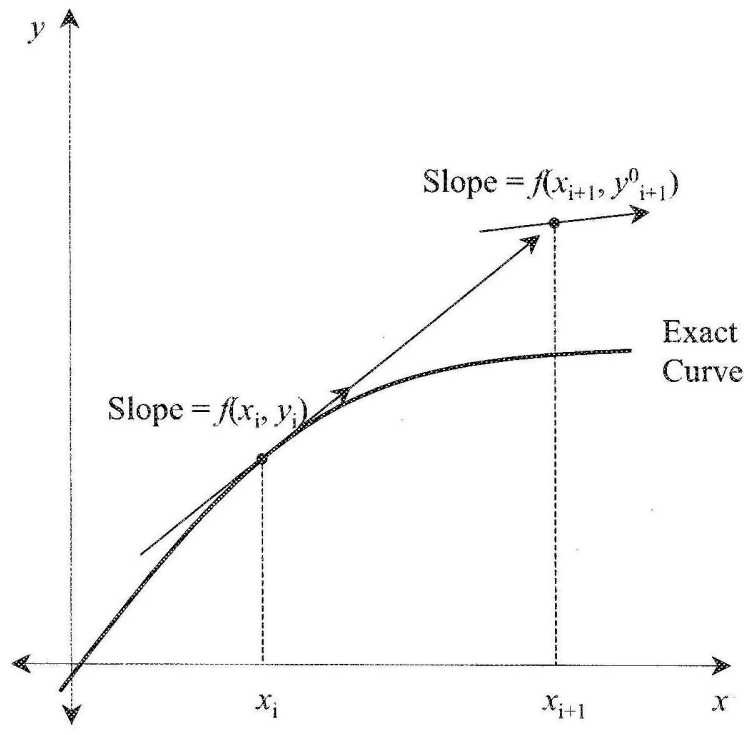

(a) Predictor

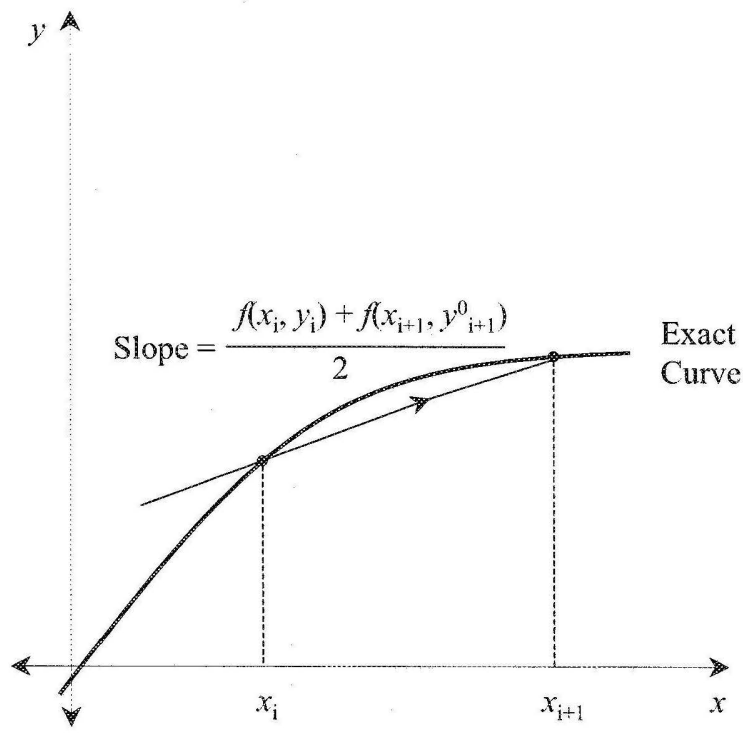

(b) Corrector

Figure 4.2: The first order implicit Euler's Predictor-Corrector method [6]

linearly to $y_{i+1}^{0}$.

$$
y_{i+1}^{0}=y_{i}+f\left(x_{i}, y_{i}\right) h
$$

where, $y_{i+1}^{0}$ represents the intermediate prediction, and $h$ is the step-size (i.e. $h=x_{i+1}-x_{i}$ ). Equation (4.36) is called a Predictor equation. It provides an estimate of $y_{i+1}^{0}$ that allows 
the calculation of an slope at the end of the interval:

$$
y_{i+1}^{\prime}=f\left(x_{i+1}, y_{i+1}^{0}\right)
$$

Two slope Equations (4.36) and (4.37) can be combined to obtain an average slope for the interval:

$$
\bar{y}^{\prime}=\frac{y_{i}^{\prime}+y_{i+1}^{\prime}}{2}=\frac{f\left(x_{i}, y_{i}\right)+f\left(x_{i+1}, y_{i+1}^{0}\right)}{2}
$$

This average slope is then used to extrapolate linearly from $y_{i}$ to $y_{i+1}$ using Euler's method $[6]$.

$$
y_{i+1}=y_{i}+\frac{f\left(x_{i}, y_{i}\right)+f\left(x_{i+1}, y_{i+1}^{0}\right)}{2}
$$

which is called a Corrector equation. Note that because Equation (4.39) has $y_{i+1}$ on the both sides of the equal sign, it can be applied in an iterative fashion. That is, an old

estimate can be used repeatedly to provide an improved estimate of $y_{i+1}$. It should be understood that this iterative process does not necessarily converge on the true answer, but will converge on an estimate with a finite truncation error, $\epsilon_{a}$, as demonstrated by following expression.

$$
\left|\epsilon_{a}\right|=\left|\frac{y_{i+1}^{j}-y_{i+1}^{j-1}}{y_{i+1}^{j}}\right| \times 100 \%
$$

where, $y_{i+1}^{j-1}$ and $y_{i+1}^{j}$ are the results from the prior and the present iteration of the corrector, respectively.

\subsubsection{Time Step-Size Control}

There are ways to estimate the local truncation error (LTE), and it can be used to adjust the step-size. In general, the strategy is to increase the step-size, if the error is small and decrease it, if the error is large. Press et al. have suggested the following criterion to 
accomplish this [87]:

$$
h_{\text {new }}=h_{\text {present }}\left|\frac{\Delta_{\text {new }}}{\Delta_{\text {present }}}\right|^{\alpha}
$$

where, $h_{\text {present }}$ and $h_{\text {new }}$ are the present and the new step sizes, respectively. $\Delta_{\text {present }}$ is the computed present accuracy, $\Delta_{n e w}$ is the desired accuracy, and $\alpha$ is a constant power that is equal to 0.2 , when the step-size is increased (that is, when $\Delta_{\text {present }} \leq \Delta_{\text {new }}$ ) and 0.25 , when the step-size is decreased $\left(\Delta_{\text {present }}>\Delta_{\text {new }}\right)$.

Constant Step-Size: In general, experiences indicate that an optimal step-size should be small enough to ensure convergence within three iterations of corrector. In addition, it must be small enough to yield a sufficiently small truncation error. At the same time, the step-size should be as large as possible to minimize runtime cost and roundoff errors. As with other methods for ODEs, the only practical way to assess the magnitude of the global error is to compare the results for the same problem but with a halved step-size.

Adaptive Step-Size: It is also called variable step-size. Two criteria are typically used to decide whether a change in step-size is warranted. First, if Equation 4.40 is greater than some prespecified error criterion, the step-size is decreased. Second, the step-size is chosen so that the convergence criterion of the corrector is satisfied in two to three iterations. The criterion is intended to account for the trade-off between the rate of convergence and the total number of steps in the calculation. For smaller step-size, the convergence will be more rapid, but more steps are required. For larger step-size, the convergence is slower, but fewer steps result. Experience suggested that the total steps will be minimized if step-size is chosen so that the corrector converges within two to three iterations. Therefore, if over two (or three) iterations are required, the step size is decreased and if less less than two (or three) iterations are required, the step-size is increased. 
Finally, the set of Equations (4.33) are solved for the unknown coefficients, $C_{j}$. Since this is a set of non-linear time dependent ordinary differential equations for $C_{j}$, they are solved simultaneously with a Newton-Raphson iterative method. The Jacobian matrix of partial derivatives is determined from $J_{i j}=\partial F_{i} / \partial C_{j}$, and the convergence is assumed to occur, when the length of the vector of the difference between two successive computed solution vector is less than $10^{-6}$. The finite difference method is used to discretize time, and a first-order implicit Euler's predictor-corrector method is used for time integration. In order to minimize the computational time without losing the accuracy at the same time, an adaptive time step controller suggested by Finlayson [88] is employed. As far as the size of mesh is concerned, a mesh of $32 \times 32$ nodes is employed in this computational study. Since each node contains four variables (refer to Equation (4.27)), the resultant Jacobian matrix is of size $4096 \times 4096$, i.e. a whopping 17 million (approx.) entries in this matrix alone. In addition, other mesh sizes used in this computational study are $21 \times 21$ and $35 \times 35$. The numerical results of this computational study were obtained from the simulations performed on SHARCNET High Performance computing Facility (CPU: $4 \times$ Opteron $@ 2.2 \mathrm{GHz} \& \mathrm{RAM}: 4.0 \mathrm{~GB})$. 


\section{CHAPTER 5}

\section{RESULTS AND DISCUSSION}

This chapter presents and discusses the simulation results obtained by solving the twodimensional mathematical model (see Equation (4.15)) representing the phase separation mechanism (i.e. TIPS via SD) in binary polymer solutions under an externally imposed spatial linear temperature gradient and also involving the thermal diffusion phenomenon. This chapter has been divided into the following three sections, which will help us understand and analyze the potential outcomes of the research thesis.

1. The Structures and Patterns Formation

2. The Time Evolution of the Dimensionless Structure Factor

3. The Transition Time from the Early Stage to the Intermediate Stage

\subsection{The Structures and Patterns Formation}

Figure 5.1 shows an asymmetric phase diagram of a binary polymer solution used in this study. The phase diagram is a plot of dimensionless temperature, $T^{*}$ versus dimensionless solvent concentration, $c^{*}$ and it has been constructed following the procedure outlined by Kurata [77]; the procedure is briefly discussed in Subsection 3.1.2, and the parameter values used in the calculation are given in Table B.1 in Appendix B. The solid curve represents the binodal curve, and the dashed curve is the spinodal curve. In addition, the three intervals $\mathrm{AB}, \mathrm{CD}$, and $\mathrm{EF}$ in the unstable region indicate the locations in the 
phase diagram for which simulation results will be presented and discussed in this chapter. These three intervals represent the overall depth of an externally imposed linear spatial temperature gradient across a polymer solution used in the simulation of the TIPS method of phase separation. Locations of these three intervals in the phase diagram highlight the main difference in $\mathrm{SD}$ pattern formation phenomena. The intervals $\mathrm{AB}$ and $\mathrm{EF}$ are at off-critical concentrations, $\left(c_{0}^{*}<c_{c}^{*}\right)$ and $\left(c_{0}^{*}>c_{c}^{*}\right)$, respectively, whereas the interval $\mathrm{CD}$ is at critical concentration, $\left(c_{0}^{*}=c_{c}^{*}\right)$. Table 5.1 lists the phase diagram coordinates $\left(c_{0}^{*}, T^{*}\right)$ of these three intervals, which represent the initial conditions used in the simulations.

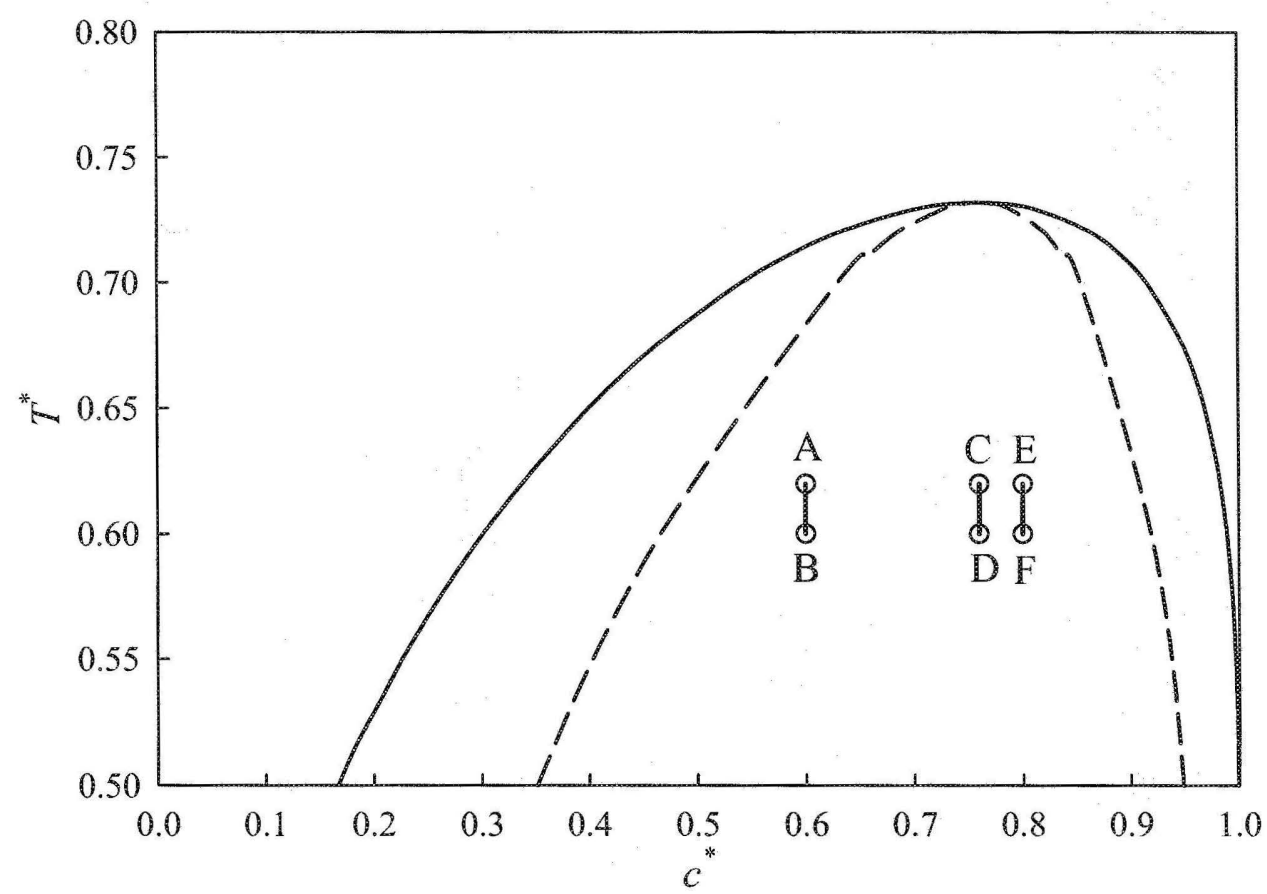

Figure 5.1: An asymmetric phase diagram for a binary polymer solution calculated from the Flory-Huggins theory using $N_{1}=1, \& N_{2}=10$. The solid curve is the binodal curve, and the dashed curve is the spinodal curve. The $c^{*}$ coordinates of the initial conditions used in the simulations are: 0.6 for interval $\mathrm{AB}$ (off-critical quench), 0.7597 for interval $\mathrm{CD}$ (critical quench) and 0.8 for interval $\mathrm{EF}$ (off-critical quench). Intervals $\mathrm{AB}, \mathrm{CD}$, and EF represent the overall depth of the spatial linear temperature gradient imposed on the polymer solution sample. 
Table 5.1: Parameter values of the selected initial conditions (see Figure 5.1)

\begin{tabular}{llll}
\hline Interval & $c_{0}^{*}$ & $T_{1}^{*}$ & $T_{2}^{*}$ \\
\hline $\mathrm{AB}$ & 0.6 & 0.6 & 0.62 \\
$\mathrm{CD}$ & 0.7597 & 0.6 & 0.62 \\
$\mathrm{EF}$ & 0.8 & 0.6 & 0.62 \\
\hline
\end{tabular}

Note that Table 5.1 represents a small sample of numerous simulations performed for the study of the phase separated structures and the pattern formation during the overall phase separation process (TIPS via SD) involving the thermal diffusion phenomenon in binary polymer solutions under an externally imposed spatial linear temperature gradient. The results, based on these simulation cases, are reflective of all the simulations performed for this study, and also, these results are sufficient to fulfil the objectives of the thesis. Figure 5.2 shows the time evolution of the dimensionless spatial concentration profile, $c^{*}\left(x^{*}, y^{*}\right)$ (first column) and patterns (second column) formed during the phase separation phenomena (TIPS via SD) involving thermal diffusion under an externally imposed spatial linear temperature gradient corresponding to interval $\mathrm{AB}$ in the phase diagram (see Figure 5.1), using a dimensionless diffusion coefficient, $D^{*}=8 \times 10^{3}$ and a dimensionless thermal diffusion coefficient, $D^{\prime *}=1.0 \times 10^{5}$. The dimensionless times are: $t^{*}=5.38 \times 10^{-5}$ (first row), $t^{*}=6.87 \times 10^{-5}$ (second row), and $t^{*}=8.01 \times 10^{-5}$ (third row). The black regions (white regions) in patterns represent solute-rich regions (solvent-rich regions) with $c^{*}<c_{c}^{*}$ $\left(c^{*}>c_{c}^{*}\right)$, where $c_{c}^{*}=0.7597$ denotes the dimensionless critical concentration of the solvent. This simulation represents pattern formation via the SD mechanism for a non-uniform offcritical quench case with a dimensionless initial average concentration, $c_{0}^{*}=0.60$, and the resultant morphology is of droplet-type. The phase separated structures consist of droplets of solvent dispersed uniformly in the solute (i.e. polymer) medium. 

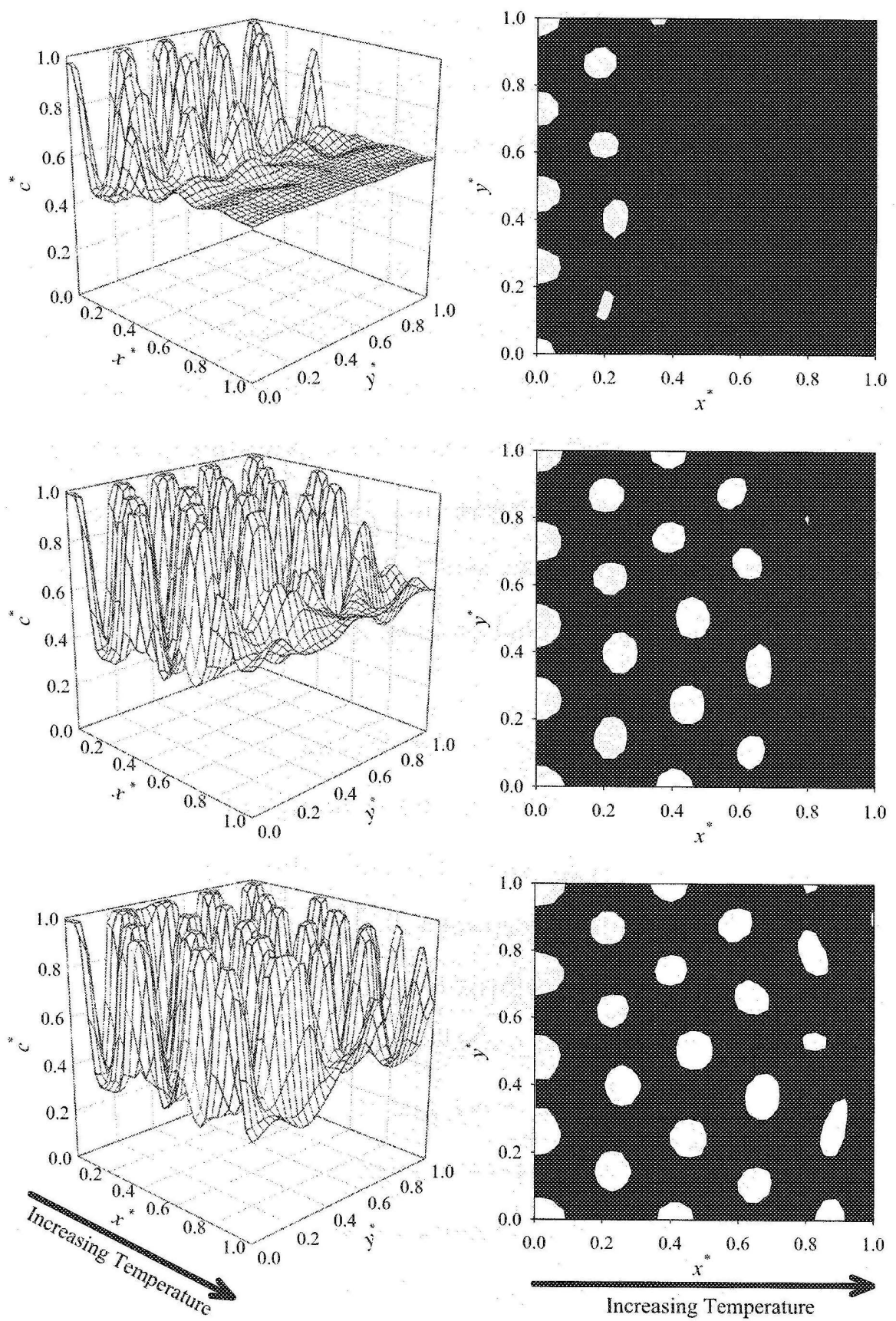

Figure 5.2: Spatial concentration profiles, $c^{*}\left(x^{*}, y^{*}\right)$ (first column) and patterns (second column) corresponding to the interval $\mathrm{AB}$ (refer to Figure 5.1) for $D^{*}=8 \times 10^{3}$ and $D^{\prime *}=1.0 \times 10^{5}$ at the following dimensionless times, $t^{*}: 5.38 \times 10^{-5}$ (first row), $6.87 \times 10^{-5}$ (second row) and $8.01 \times 10^{-5}$ (third row). The black regions (white regions) in patterns represent solute-rich regions (solvent-rich regions) with $c^{*}<c_{c}^{*}\left(c^{*}>c_{c}^{*}\right)$, where $c_{c}^{*}=0.7597$ denotes the dimensionless critical concentration. The figure corresponds to a non-uniform off-critical quench case with $c_{0}^{*}=0.60$, and the resultant pattern is of droplet-type. 
Figure 5.3 shows the time evolution of the dimensionless spatial concentration profile, $c^{*}\left(x^{*}, y^{*}\right)$ (first column) and patterns (second column) formed during the phase separation phenomena (TIPS via SD) involving thermal diffusion under an externally imposed spatial linear temperature gradient corresponding to interval CD in the phase diagram (see Figure 5.1), using a dimensionless diffusion coefficient, $D^{*}=8 \times 10^{3}$ and a dimensionless thermal diffusion coefficient, $D^{\prime \prime *}=1.0 \times 10^{5}$. The dimensionless times are: $t^{*}=2.98 \times 10^{-5}$ (first row), $t^{*}=3.44 \times 10^{-5}$ (second row), and $t^{*}=6.93 \times 10^{-5}$ (third row). The black regions (white regions) in patterns represent solute-rich regions (solvent-rich regions) with $c^{*}<c_{c}^{*}\left(c^{*}>c_{c}^{*}\right)$. This simulation represents pattern formation via the SD mechanism for a non-uniform critical quench case with a dimensionless initial average concentration, $c_{0}^{*}=0.7597$, and the resultant morphology is of transient interconnected-type structures.

Figure 5.4 shows the time evolution of the dimensionless spatial concentration profile $c^{*}\left(x^{*}, y^{*}\right)$ (first column) and patterns (second column) formed during the phase separation (TIPS via SD) phenomena involving thermal diffusion under an externally imposed spatial linear temperature gradient corresponding to interval EF in the phase diagram (see Figure 5.1), using a dimensionless diffusion coefficient, $D^{*}=8 \times 10^{3}$ and a dimensionless thermal diffusion coefficient, $D^{\prime *}=1.0 \times 10^{5}$. The dimensionless times are: $t^{*}=6.68 \times 10^{-5}$ (first row), $t^{*}=7.86 \times 10^{-5}$ (second row), and $t^{*}=10.58 \times 10^{-5}$ (third row). The black regions (white regions) in patterns represent solute-rich regions (solvent-rich regions) with $c^{*}<c_{c}^{*}\left(c^{*}>c_{c}^{*}\right)$. This simulation also represents pattern formation via the SD mechanism for a non-uniform off-critical quench case with a dimensionless initial average concentration, $c_{0}^{*}=0.80$, and the resultant morphology is again of droplet-type. But, unlike the simulation results corresponding to the interval $\mathrm{AB}$, the phase separated structures, formed in the simulation corresponding to the interval EF, consist of droplets of solute (i.e. polymer) dispersed uniformly in the solvent medium (reversal of black and white regions, refer to Figures 5.2 and 5.4). 

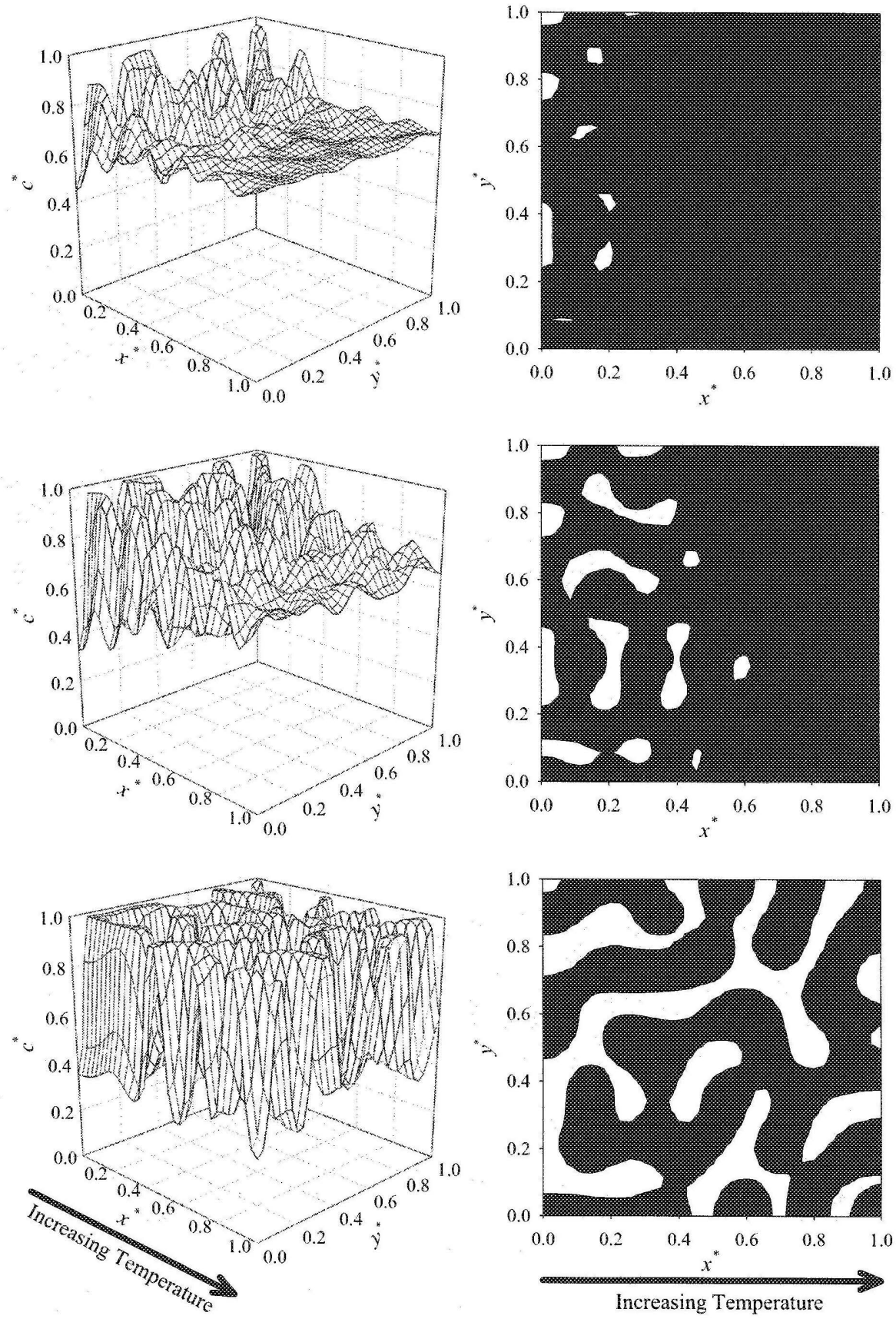

Figure 5.3: Spatial concentration profiles, $c^{*}\left(x^{*}, y^{*}\right)$ (first column) and patterns (second column) corresponding to the interval CD (refer to Figure 5.1) for $D^{*}=8 \times 10^{3}$ and $D^{\prime *}=$ $1.0 \times 10^{5}$ at the following dimensionless times, $t^{*}: 2.98 \times 10^{-5}$ (first row), $3.44 \times 10^{-5}$ (second row) and $6.93 \times 10^{-5}$ (third row). The black regions (white regions) in patterns represent solute-rich regions (solvent-rich regions) with $c^{*}<c_{c}^{*}\left(c^{*}>c_{c}^{*}\right)$, where $c_{c}^{*}=0.7597$ denotes the dimensionless critical concentration. The figure corresponds to a non-uniform critical quench case with $c_{0}^{*}=0.7597$, and the resultant pattern is of transient interconnected-type structures. 

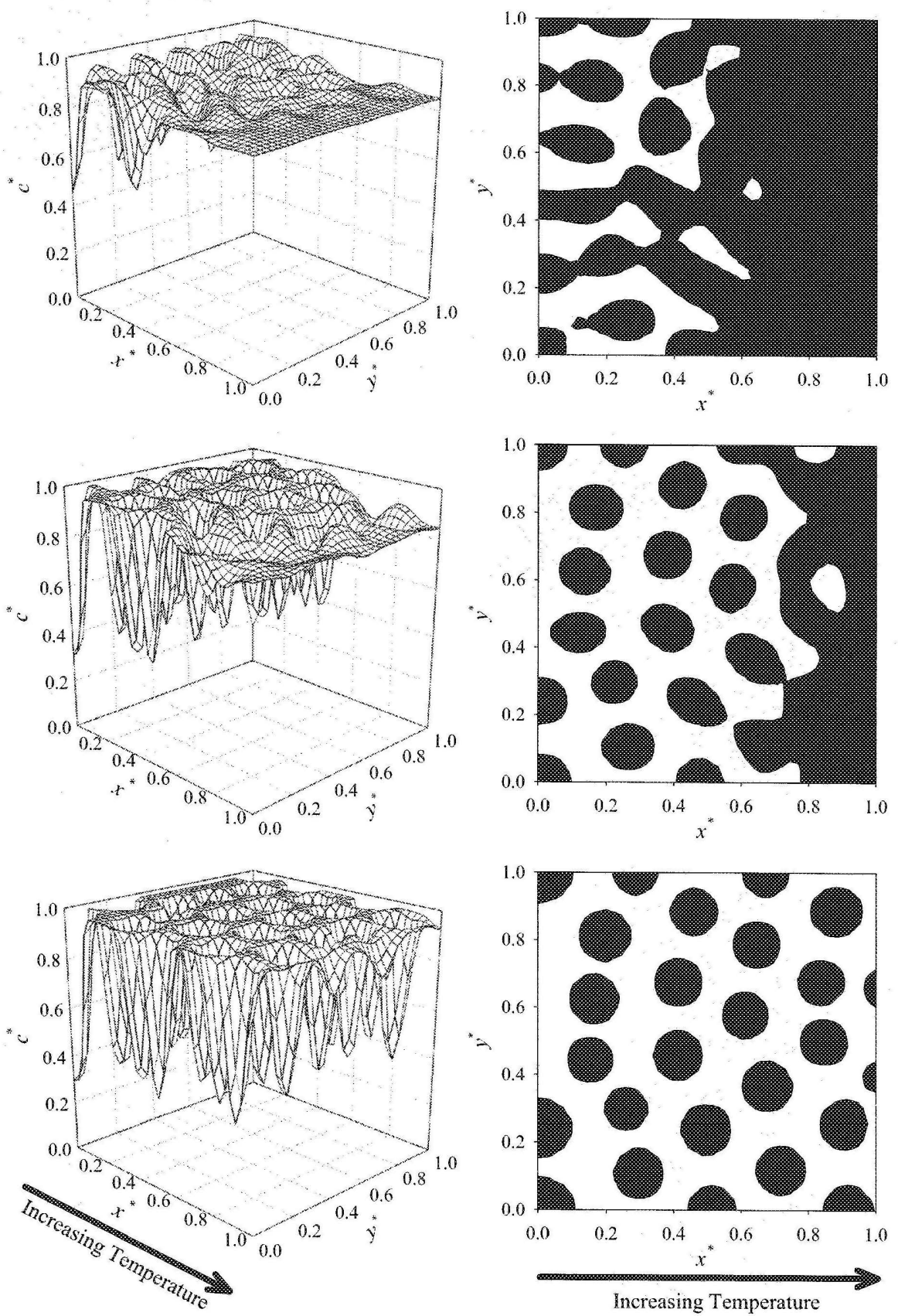

Figure 5.4: Spatial concentration profiles, $c^{*}\left(x^{*}, y^{*}\right)$ (first column) and patterns (second column) corresponding to the interval EF (refer to Figure 5.1) for $D^{*}=8 \times 10^{3}$ and $D^{\prime *}=1.0 \times 10^{5}$ at the following dimensionless times, $t^{*}: 6.68 \times 10^{-5}$ (first row), $7.86 \times 10^{-5}$ (second row) and $10.58 \times 10^{-5}$ (third row). The black regions (white regions) in patterns represent solute-rich regions (solvent-rich regions) with $c^{*}<c_{c}^{*}\left(c^{*}>c_{c}^{*}\right)$, where $c_{c}^{*}=0.7597$ denotes the dimensionless critical concentration. The figure corresponds to a non-uniform critical quench case with $c_{0}^{*}=0.80$, and the resultant pattern is of droplet-type. 
Now, analyzing the simulation results presented by Figures 5.2, 5.3 and 5.4, it can be depicted that during the phase separation mechanism of TIPS via SD involving the thermal diffusion phenomenon under an externally imposed spatial linear temperature gradient for non-uniform off-critical quench cases (intervals $\mathrm{AB}$ and $\mathrm{EF}$ in Figure 5.1), the resultant pattern or morphology formation is of droplet-type, whereas for a non-uniform critical quench case (interval CD in Figure 5.1), the resultant pattern or morphology formation is of interconnected-type structures. It should be noted that the pattern or morphology formations in the simulation cases studies in this work, are anisotropic in nature, which is due to the fact that the phase separation is induced by non-uniform (under spatial linear temperature gradient) quenching of the polymer solution. The simulation results describing the formation of droplet-type morphology at intervals $\mathrm{AB}$ (Figure 5.2) and EF (Figure 5.3) (off-critical non-uniform quench cases) and the formation of the interconnected-type structures at interval CD (Figure 5.4) (critical non-uniform quench case) are consistent with the experimental results presented by Kyu et al [89] and Tanaka et al [90]. In addition, these results are also in good agreement with the numerical results presented by Copetti and Elliott [91], Chakrabarti [92], Brown and Chakrabarti [93], Chan and Rey [28,33], and Jiang and Chan [19]. Moreover, analyzing the spatial concentration profiles and patterns in Figures 5.2, 5.3 and 5.4, it is interesting to note that the initiation of the phase separation phenomena begins at the low temperature region of the polymer solution sample at early times, and the phase separated regions grow over time increasingly from the region, $x^{*}=0$ to $x^{*}=1$ to occupy the entire solution sample area. This kind of morphological development is due to the externally imposed spatial linear temperature gradient across the polymer solution sample, which induces the effect of differential quench along the $x^{*}$ direction. At low temperature region, i.e. close to $x^{*}=0$, small droplets are formed initially at early stage due to the deep quench effect and become larger at later times due to coarsening in the late stage, whereas at the high temperature region, i.e. close to $x^{*}=1$, fairly large droplets are formed initially at early stage due to the shallow quench effect, 
and that is why the resultant pattern or morphology formation is of anisotropic in nature. And also, since the droplets are formed at different times due to the difference in quench depth across the polymer solution sample in $x^{*}$-direction, the droplets will undergo the three stages (i.e. the early stage, the intermediate stage and the late stage, refer to Figure 1.4) of SD at different times [22]. But, after certain period of time of phase separation, the droplets size in both low and high temperature regions becomes apparently uniform, as it is evident from Figures 5.2 and 5.4. These anisotropic morphological results are in good agreement with the experimental results presented by Matsuyama et al $[16,56]$ and numerical results presented by Lee et al [22]. Other important phenomenon, which could be contributing to the above mentioned morphological development, is the thermal diffusion phenomenon or Ludwig-Soret effect. As discussed earlier, a spatial temperature gradient applied to a fluid mixture generally induces net mass flows across the mixture, which leads to the formation of concentration gradients at microscopic level in the mixture. This cross effect between temperature and concentration gradient is known as Ludwig-Soret effect or thermal diffusion phenomenon. These microscopically induced concentration gradients also increase the total free energy of the solution sufficiently to enhance the phase separation process in a polymer solution by the spinodal decomposition (SD) mechanism, as the initial conditions of the solutions are maintained such that the quench interval of the solution always lies in the spinodal region of the phase diagram (see Figure 5.1 and Table $5.1)$.

Apparently, it is very difficult to visualize the effect of the thermal-diffusion on the overall phase separation mechanism in a polymer solution, as there are no visual differences in the pattern or morphological development with or without consideration of the thermaldiffusion phenomenon at microscopic level. Therefore, in order to identify the effect of the thermal diffusion phenomenon on the overall phase separation mechanism (TIPS via $\mathrm{SD}$ ) in a polymer solution microscopically, the time evolution of the dimensionless struc- 
ture factor and the dimensionless transition time can be utilized, which will help us better understand the characteristics of the phase separation mechanism in a polymer solution.

\subsection{Time Evolution of Dimensionless Structure Factor}

The dimensionless structure factor is an important parameter used to characterize the development of phase separated domains by the SD mechanism in polymer solutions or blends, and it helps relate the numerical results and experimental studies performed on the phase separation mechanism in polymer solutions. Therefore, the time evolution of the twophase structures, such as ones produced during the phase separation process in the polymer systems studied in this work, can also be quantified using the dimensionless structure factor, $S^{*}\left(\mathbf{k}^{*}, t^{*}\right)$. Before, we jump onto the dimensionless structure factor calculation, it is very important to understand the relationship between the dimensionless structure factor, $S^{*}\left(\mathbf{k}^{*}, t^{*}\right)$ and the scattered light intensity, $I_{s}$ in the small angle light scattering (SALS) experiments, which are carried out to study the extent of phase separation in polymer solutions or blends. Mathematically, the relationship between the scattered light intensity, $I_{s}$ in small angle light scattering (SALS) experiments and the dimensionless structure factor, $S^{*}\left(\mathbf{k}^{*}, t^{*}\right)$ in the numerical study for the phase separation mechanism in polymer solutions or blends is of following form $[91,94]$.

$$
I_{s}(q, t) \propto S^{*}\left(\mathbf{k}^{*}, t^{*}\right)=\left|A^{*}\left(\mathbf{k}^{*}, t^{*}\right)\right|^{2} \quad \text { for } \mathbf{k}^{*}=q
$$

Where, $I_{s}$ is the scattered light intensity, $q$ is the scattering wave vector or wave number, $S^{*}\left(\mathbf{k}^{*}, t^{*}\right)$ is the dimensionless structure factor, $\mathbf{k}^{*}$ is the two-dimensional position vector, and $A^{*}\left(\mathbf{k}^{*}, t^{*}\right)$ is the magnitude of the Fourier transform of the concentration fluctuations in the system. Thus, experimental light scattering data is directly proportional to the dimensionless structure factor and a detailed discussion of the development of this expression 
(Equation (5.1)) can be found elsewhere [94]. The following summarizes the characteristics of the early stage to the intermediate stage of phase separation by the SD mechanism in relation to the light scattering data obtained from the SALS experiments [7].

Early Stage: According to the linear Cahn theory [78] in the early stage of SD, $I_{s}$ can be related with time $t$ as:

$$
I_{s}(q, t) \propto I_{s}(q, t=0) \exp [2 R(q) t]
$$

where, $R(q)$ is a growth rate of the concentration fluctuation. Wave number, $q$ is given by following expression:

$$
q=\left(\frac{4 \pi n}{\lambda_{0}}\right) \sin \left(\frac{\theta}{2}\right)
$$

Here, $n$ is the solution refractive index, and $\lambda_{0}$ is the wavelength of light in vacuo. $R(q)$ can be correlated to $q$ as:

$$
R(q)=D_{\text {app }} q^{2}\left[1-\frac{q^{2}}{2 q_{m}^{2}}\right]
$$

where, $D_{\text {app }}$ is the apparent diffusion coefficient, and $q_{m}$ is the wave number of maximum scattered light intensity. Equation (5.2) implies that the scattered light intensity, $I_{s}$ grows exponentially during the early stage of the phase separation by the SD mechanism and the growth rate of the concentration fluctuation, $R(q)$ is weakly non-linear as can be observed from Equation (5.4). Therefore, a plot of natural log of the scattered light intensity given by Equation (5.2) should produce a straight line for the early stage of phase separation by the SD mechanism.

Intermediate Stage: During the intermediate stage of the phase separation by the SD mechanism, the scattered light intensity continues to increase, but at a slower rate 
than in the early stage of phase separation by the SD mechanism. And also, in the intermediate stage, the wave number $q$ decreases and the wavelength $\lambda$ increases, which is due the fact that $q \propto \frac{1}{\lambda}$ (see Equation (5.3)).

A typical plot of the scattered light intensity, $I_{s}$ versus the wave number, $q$ in the early to the beginning of the intermediate stages for phase separation by the SD mechanism is shown in Figure 5.5.

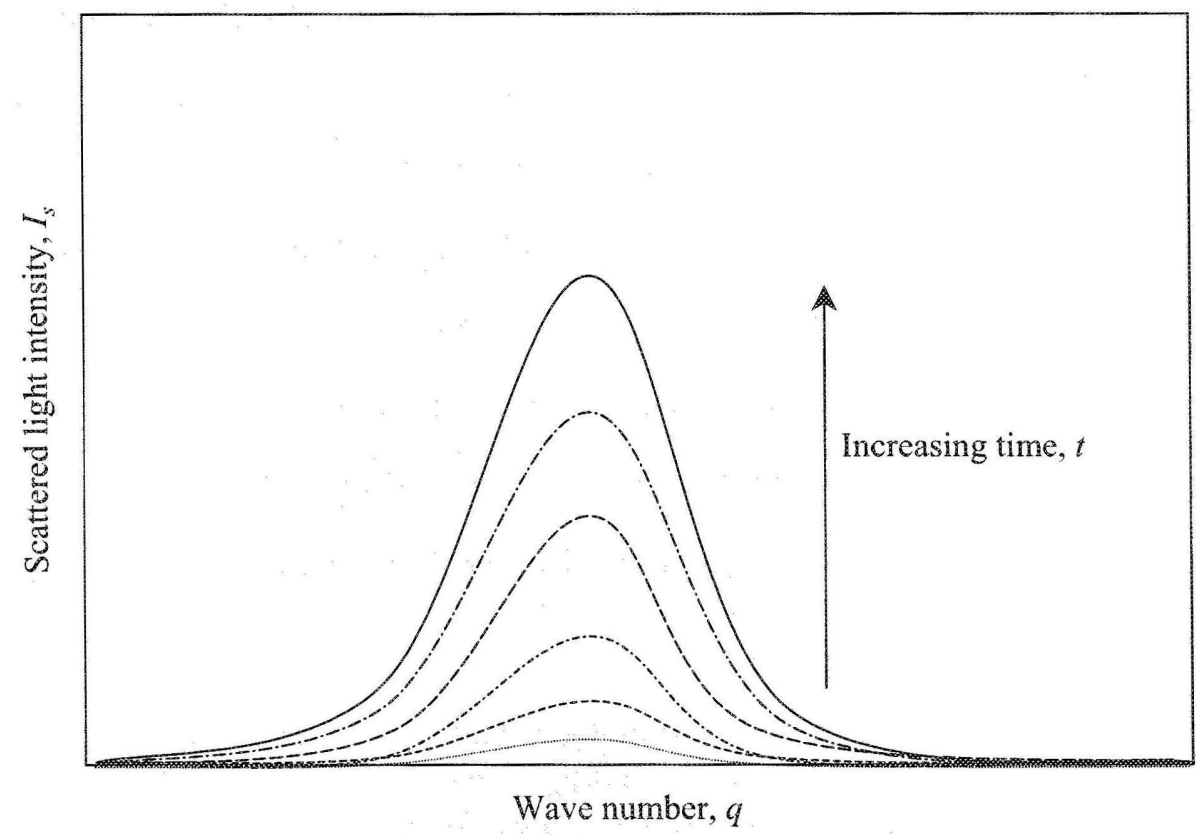

Figure 5.5: Typical light scattering profiles showing the time evolution of phase separation by the SD mechanism in the early stage to the beginning of the intermediate stage in the small angle light scattering (SALS) experiment. Each profile represents scattered light intensity at certain time, $t$. The scattered light intensity, $I_{s}$ is increasing with time, while the wave number, $q$ remains constant, which is typical characteristic of the early stage of $\mathrm{SD}[7]$.

Thus, in summary, the dimensionless structure factor, $S^{*}\left(\mathbf{k}^{*}, t^{*}\right)$ and the dimensionless wave (position) vector, $\mathbf{k}^{*}$ closely resemble the scattered light intensity, $I_{s}(q, t)$ and wave number, $q$ in the small angle light scattering (SALS) experiments performed for the phase separation studies in polymer mixtures, respectively (refer to Equation (5.1)). The dimensionless 
structure factor, $S^{*}\left(\mathbf{k}^{*}, t^{*}\right)$ is calculated simply by taking the squares of the magnitude of the Fourier transform, $A^{*}\left(\mathbf{k}^{*}, t^{*}\right)$ of the concentration fluctuations in the system. Therefore, from Equation (5.1), the dimensionless structure factor can be expressed as:

$$
S^{*}\left(\mathbf{k}^{*}, t^{*}\right)=\left|A^{*}\left(\mathbf{k}^{*}, t^{*}\right)\right|^{2}=\left|\sum_{m=0}^{N-1} \sum_{n=0}^{N-1}\left(c_{m, n}-c_{0}\right) \exp \left(\frac{2 \pi i}{N}\left(m k_{x}+n k_{y}\right)\right)\right|^{2}
$$

where, $c_{m, n}$ is the concentration of solvent at node, $(m, n), c_{0}$ is the mean solvent concentration, and $\mathbf{k}^{*}$ is the two-dimensional position vector, $\left(k_{x}, k_{y}\right)$ in Fourier space. In order to calculate the dimensionless structure factor, $S^{*}\left(\mathbf{k}^{*}, t^{*}\right)$, a small computer program written in MATLAB R2007a was used to determine the Fast Fourier Transform (FFT), $A^{*}\left(\mathbf{k}^{*}, t^{*}\right)$ of the simulation results (sample data) at a specific time, $t^{*}$ and then taking the square of this FFT results yields the dimensionless structure factor. Table 5.2 lists the dimensionless parameters used in four simulation cases considered for the investigation of the effect thermal diffusion phenomenon during the phase separation mechanism in binary polymer solutions under an externally imposed spatial linear temperature gradient.

Table 5.2: Dimensionless material parameters used in the simulation study of the investigation of the effect of thermal diffusion phenomenon on TIPS via the SD mechanism under an externally imposed spatial linear temperature gradient in binary polymer solutions.

\begin{tabular}{|c|c|c|c|c|c|c|c|c|}
\hline \multirow{2}{*}{ Case } & \multicolumn{7}{|c|}{ Dimensionless Parameters } \\
\cline { 2 - 9 } & $N_{\mathbf{1}}$ & $N_{\mathbf{2}}$ & $c_{0}^{*}$ & $D^{*}$ & $D^{\prime *}$ & $\psi$ & $T_{1}^{*}$ & $T_{2}^{*}$ \\
\hline 1 & 1 & 10 & 0.55 & $8.0 \times 10^{3}$ & 0 & 1.0 & 0.60 & 0.62 \\
\hline 2 & 1 & 10 & 0.55 & $8.0 \times 10^{3}$ & $1.0 \times 10^{5}$ & 1.0 & 0.60 & 0.62 \\
\hline 3 & 1 & 10 & 0.55 & $8.0 \times 10^{3}$ & $3.0 \times 10^{5}$ & 1.0 & 0.60 & 0.62 \\
\hline 4 & 1 & 10 & 0.55 & $8.0 \times 10^{3}$ & $5.0 \times 10^{5}$ & 1.0 & 0.60 & 0.62 \\
\hline
\end{tabular}


Table 5.2 represents a small sample of numerous simulations performed in this study for the investigation of the effect of thermal diffusion phenomenon or the Ludwig-Soret effect on the overall phase separation mechanism in binary polymer solutions and the results, based on these simulations, are sufficient to fulfil the objectives of the thesis. Figures 5.6, $5.7,5.8$ and 5.9 show the time evolution of the dimensionless structure factor (plot of structure factor, $S^{*}\left(\mathbf{k}^{*}, t^{*}\right)$ vs wave vector, $\left.\mathbf{k}^{*}\right)$ for the simulations corresponding to the thermal diffusion coefficients: $D^{\prime *}=0($ Case 1$), D^{\prime *}=1.0 \times 10^{5}($ Case 2$), D^{\prime *}=3.0 \times 10^{5}$ (Case 3) and $D^{\prime *}=5.0 \times 10^{5}$ (Case 4 ), respectively (refer to Table 5.2 for other parameter values).

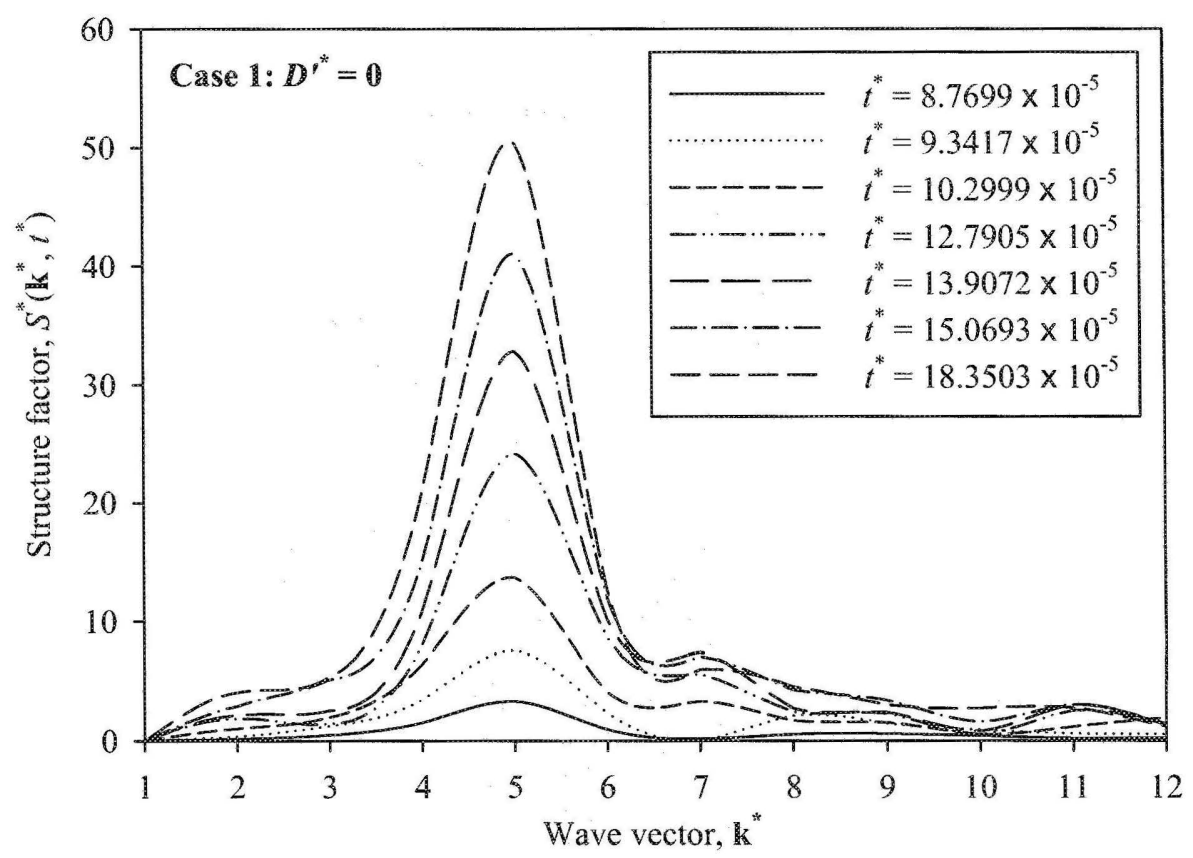

Figure 5.6: The time evolution of the dimensionless structure factor, $S^{*}\left(\mathbf{k}^{*}, t^{*}\right)$ for the simulation corresponding to the thermal diffusion coefficient, $D^{\prime *}=0$ (refer to case 1 in Table 5.2 for other parameter values).

Referring to these Figures 5.6 to 5.9 , it can be observed that the value of the dimensionless structure factor increases exponentially with time during the early stage of the phase separation by the SD mechanism and reaches maximum as it approaches beginning of the 


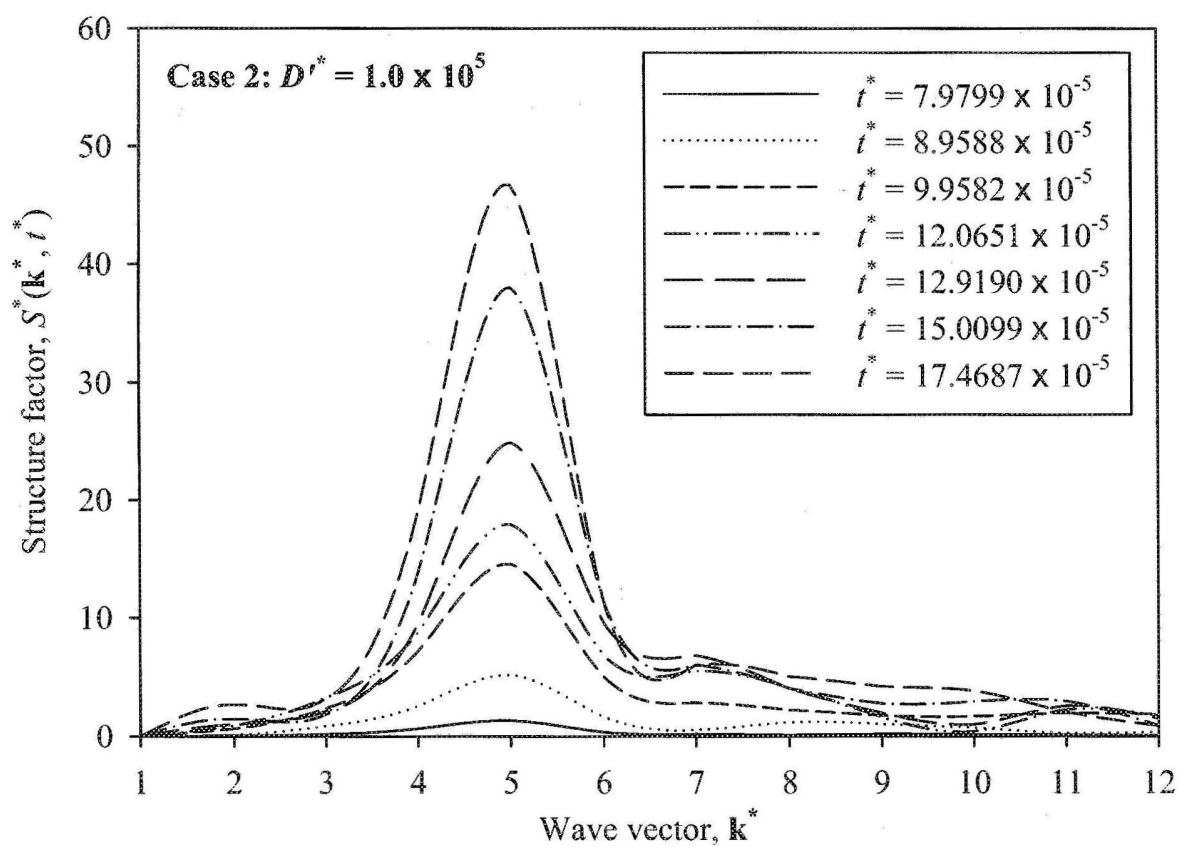

Figure 5.7: The time evolution of the dimensionless structure factor, $S^{*}\left(\mathbf{k}^{*}, t^{*}\right)$ for the simulation corresponding to the thermal diffusion coefficient, $D^{\prime *}=1.0 \times 10^{5}$ (refer to case 2 in Table 5.2 for other parameter values).

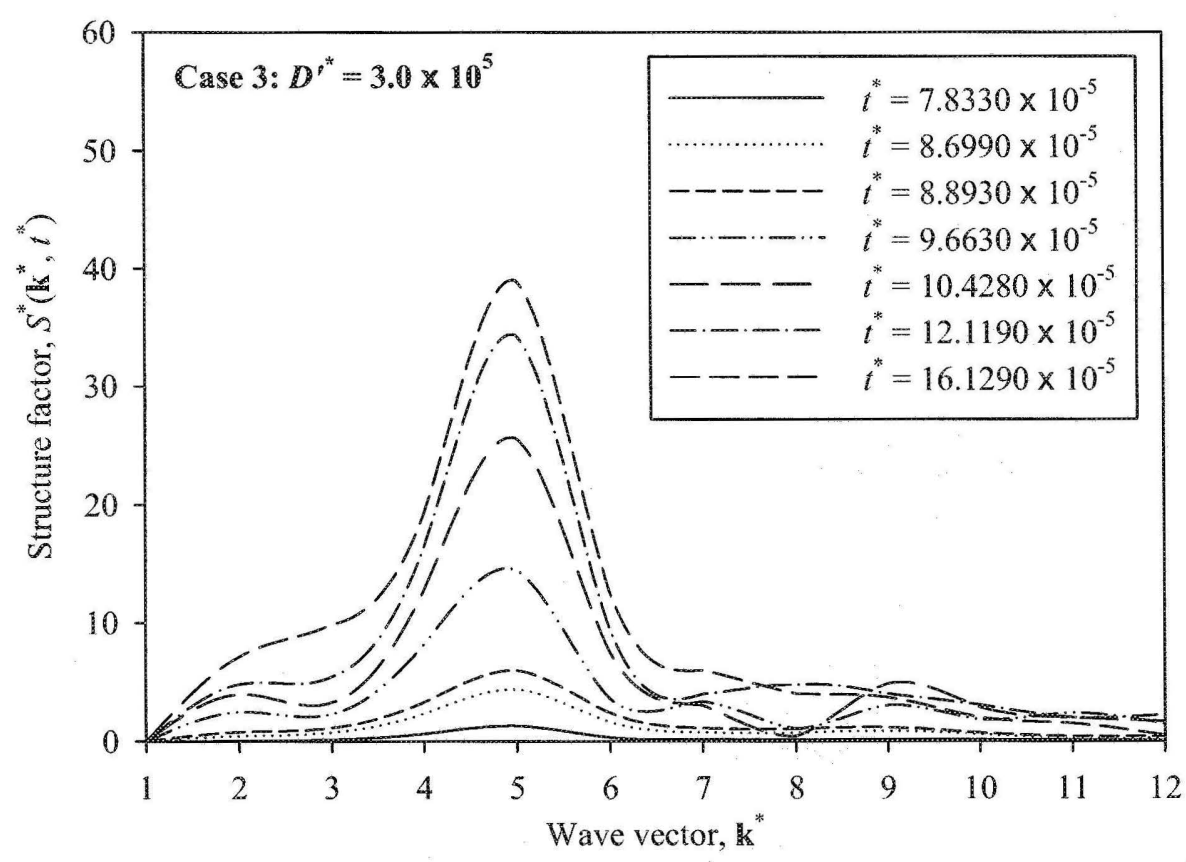

Figure 5.8: The time evolution of the dimensionless structure factor, $S^{*}\left(\mathbf{k}^{*}, t^{*}\right)$ for the simulation corresponding to the thermal diffusion coefficient, $D^{\prime *}=3.0 \times 10^{5}$ (refer to case 3 in Table 5.2 for other parameter values). 


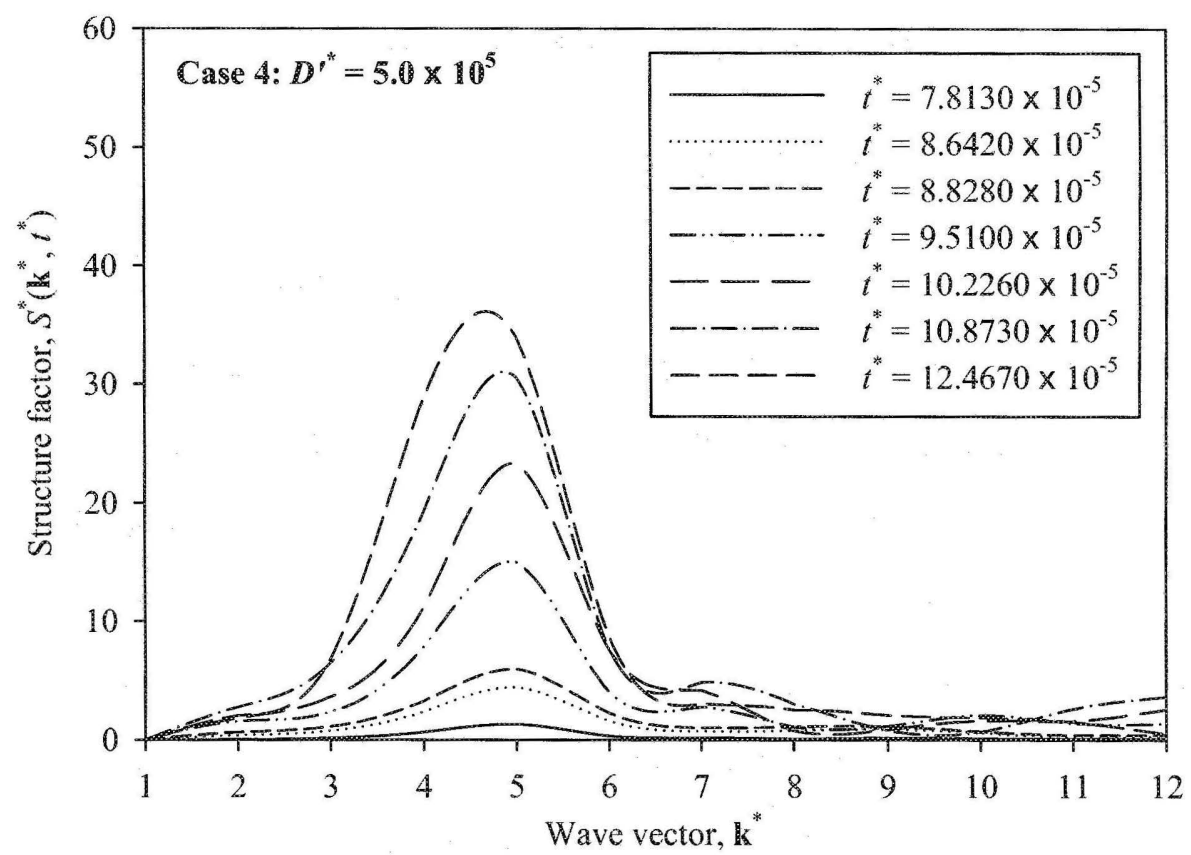

Figure 5.9: The time evolution of the dimensionless structure factor, $S^{*}\left(\mathbf{k}^{*}, t^{*}\right)$ for the simulation corresponding to the thermal diffusion coefficient, $D^{\prime *}=5.0 \times 10^{5}$ (refer to case 4 in Table 5.2 for other parameter values).

intermediate stage. It is also interesting to note that the wave vector remains constant in all the four cases shown during the phase separation process in the early to the beginning of the intermediate stages, which is quite normal trend during the early stage of the phase separation mechanism [7] and is consistence with the results reported in the experimental $[62,63]$ as well as numerical [34] works. It can also be noted that as magnitude of the dimensionless thermal diffusion coefficient increases, the time to reach the maximum structure factor $S^{*}\left(\mathbf{k}_{m}^{*}, t^{*}\right)$ decreases, i.e the phase separation mechanism proceeds at a faster rate. Thus, it can be concluded from these findings that the thermal diffusion phenomenon does have some sort of the impact on the extent of phase separation mechanism as it speeds up the overall phase separation process at microscopic level, but not significantly. 


\subsection{The Transition Time from the Early Stage to the Intermediate Stage}

Another important characteristic parameter, called the dimensionless transition time, $t_{t}^{*}$, can also be used to characterize the extent of polymer phase separation process by the SD mechanism. It will also help us analyze the effect of the dimensionless thermal diffusion coefficient, $D^{\prime *}$ on the overall process of polymer phase separation (i.e. TIPS) by the SD mechanism. As discussed in Chapter 1, the overall phase separation process by the SD mechanism is comprised of three stages: the early stage, the intermediate stage, and the late stage. The transition time basically represents the dimensionless time of transition between the early stage and the intermediate stage of the phase separation process by SD.

Figure 5.10 shows a plot of the natural $\log$ of the dimensionless maximum structure factor, $S^{*}\left(\mathbf{k}_{m}^{*}, t^{*}\right)$ versus the dimensionless time, $t^{*}$ for a non-uniform quench case of polymer phase separation mechanism (TIPS via SD) in polymer solutions. This curve is typical of the spinodal decomposition (SD) mechanism, since there is an exponential growth initially at early stage, and then it slows down when it enters the intermediate stage of the phase separation mechanism. The intersection of the two straight lines drawn on each side of the curve represents the transition point between the early and the intermediate stages of the SD mechanism and the dimensionless time indicated by the downward arrow gives the dimensionless transition time, $t_{t}^{*}$ (refer to Figure 5.10). Table 5.3 lists the calculated values of the dimensionless transition time, $t_{t}^{*}$ corresponding to the simulation cases listed in $\mathrm{Ta}-$ ble 5.2 with respect to the different thermal diffusion coefficients. It can be observed from these results that as the value of the dimensionless thermal diffusion coefficient increases, the value of dimensionless transition time decreases very little in magnitude comparatively, which is again indicative of the fact that the thermal diffusion phenomenon or the LudwigSoret effect does have some influence on the phase separation mechanism (TIPS via SD) 
in polymer solutions, as the phase separation process proceeds at relatively faster rate in presence of the thermal diffusion phenomenon in polymer solutions under an externally imposed spatial linear temperature gradient.

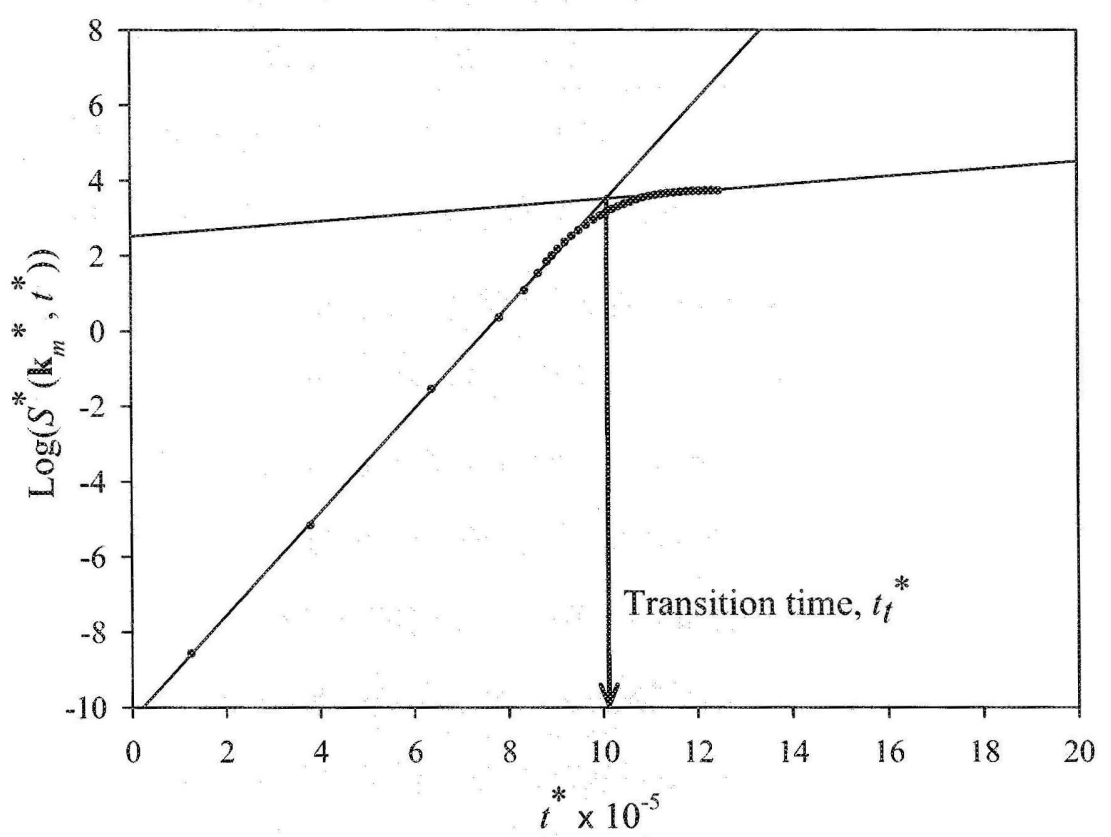

Figure 5.10: A semilog plot of the time evolution of the dimensionless maximum structure factor, $S^{*}\left(\mathbf{k}_{m}^{*}, t^{*}\right)$ versus the dimensionless time, $t^{*}$ for a non-uniform quench case of polymer phase separation mechanism (TIPS via SD) in polymer solutions. This curve is typical of the spinodal decomposition (SD) mechanism, since there is an exponential growth initially at early stage, and then it slows down when it enters the intermediate stage of the phase separation mechanism.

In summary, the pattern or morphology formation during the polymer phase separation mechanism (TIPS via SD) in polymer solutions involving the thermal diffusion phenomenon under an externally imposed spatial linear temperature gradient is of droplet-type in cases of non-uniform off-critical quench, i.e. $\left(c_{0}^{*}<c_{c}^{*}\right.$ and $\left.c_{0}^{*}>c_{c}^{*}\right)$, and of interconnected-type structures in case of non-uniform critical quench, i.e. $\left(c_{0}^{*}=c_{c}^{*}\right)$. And also, it is interesting 
Table 5.3: Transition time, $t_{t}^{*}$ corresponding to the simulation cases listed in Table 5.2.

\begin{tabular}{ccc}
\hline Case & $D^{\prime *}$ & $t_{t}^{*} \times 10^{-5}$ \\
\hline Case 1 & 0 & 10.0061 \\
Case 2 & $1.0 \times 10^{5}$ & 9.9858 \\
Case 3 & $3.0 \times 10^{5}$ & 9.9045 \\
Case 4 & $5.0 \times 10^{5}$ & 9.6850 \\
\hline
\end{tabular}

to note that both the morphologies, the droplet-type as well as the interconnected-type structures are of anisotropic in nature as the phase separation is induced by non-uniform (under temperature gradient) quenching of the polymer solution. These results are consistent with the experimental as well as the numerical results reported in the literature (refer to Section 5.1). As far as the effect of thermal diffusion on the pattern or morphology formation concerned, there are no visual morphological differences during the phase separation mechanism with or without the consideration of the thermal diffusion phenomenon. On the other hand, at microscopic level, the thermal diffusion phenomenon or the LudwigSoret effect has very little or negligible effect on the phase separation mechanism (TIPS via SD) in polymer solutions under non-uniform temperature field in the early to the intermediate stages of the SD as expected, which is evident from the studies of the time evolution of the dimensionless structure factor (refer to Section 5.2) and the dimensionless transition time (refer to Section 5.3). 


\section{CHAPTER 6 \\ CONCLUSIONS}

Thermal-induced phase separation (TIPS) method is one the most widely used methods for the manufacturing of the functional polymeric materials, i.e. PDLC films for electrooptical devices, microporous synthetic membranes, etc. Also, the induced temperature gradient for producing the structural anisotropy in the polymer films. A rigorous mathematical model, representing the phase separation mechanism, i.e. TIPS via the spinodal decomposition (SD) mechanism, and also involving the thermal diffusion phenomenon in binary polymer solutions under an externally imposed spatial linear temperature gradient, was developed and solved successfully. The mathematical model is composed of the nonlinear Cahn-Hilliard theory describing the dynamic behavior of the spinodal decomposition (SD) mechanism, the Flory-Huggins (F-H) theory describing thermodynamics of polymer solutions, the slow mode theory and Rouse law for diffusion phenomena in polymer solutions, and the theory of Ludwig-Soret effect describing the thermal diffusion phenomenon in polymer solutions under the influence of an externally imposed spatial temperature gradient. The mathematical model was solved using the numerical methods of Galerkin finite elements with Hermitian bicubic interpolants and finite differences, respectively for spatial and temporal discretizations. The first-order implicit Euler predictor-corrector method was used for time integration; and the time steps were controlled using an adaptive time-step control mechanism on the local truncation error and tolerance in such a manner that a given accuracy was always maintained. 
The mathematical model was successfully implemented to study the Ludwig-Soret effect in the phase separation mechanism (TIPS via SD) involving the thermal diffusion phenomenon in binary polymer solutions under an externally imposed spatial linear temperature gradient in one-dimension as well in two-dimensions, although only two-dimensional simulation results have been presented and discussed in this work. In order to investigate the effect of thermal diffusion phenomenon on the phase separation mechanism (TIPS via SD), two kinds of the quench methods (i.e. uniform and non-uniform) to induce the phase separation in single phase homogeneous polymer solutions, were studied for comparison. For uniform quench, a single phase homogeneous polymer solution is quenched to a temperature, which is uniform everywhere in the solution, i.e. no temperature gradients. Whereas, for non-uniform quench, polymer solution is quenched in such a way that a spatial temperature gradient is formed in the solution. The simulation results represented by the pattern or morphology formation during the phase separation process of TIPS via SD in a binary polymer solution involving the thermal diffusion phenomenon under an externally imposed spatial linear temperature gradient indicated that non-uniform off-critical quenching of the polymer solution produced the droplet-type morphology, whereas a non-uniform critical quenching of the polymer solution produced the transient interconnected-type structures, which are of anisotropic in nature. These simulation results describing the formation of the droplet-type morphologies (off-critical quench) and the interconnected-type structures (critical quench) were consistent with the ones reported in the literature. And also, the externally imposed spatial linear temperature gradient contributed to the formation of the anisotropic morphological structures, which were also found to be consistent with the experimental as well as numerical results presented in the literature. As far as the effect of thermal diffusion phenomenon on the phase separation mechanism (TIPS via SD) was concerned, it was very difficult to identify the effect of thermal diffusion on the pattern formation during the phase separation process, as there were no visual differences in the patterns formation with or without the consideration of thermal diffusion phenomenon. 
Therefore, in order to investigate the influence of the thermal diffusion phenomenon on the phase separation mechanism in polymer solutions at microscopic level, the time evolution of the dimensionless structure factor and the transition time were analyzed. The dimensionless structure factor was calculated by taking the squares of the magnitude of the Fourier Transform of the concentration fluctuations in the polymer solution. For that a small computer program written in MATLAB R2007a was used to calculate the Fast Fourier Transform (FFT) of the simulation results (sample data) at specific time, and the squares of these FFT results yielded the dimensionless structure factor. It was evident from the time evolution of the dimensionless structure factor and the transition time that the thermal diffusion phenomena actually had very little or negligible influence on the phase separation mechanism (TIPS via SD) during the early to the intermediate stages of $\mathrm{SD}$, although it assisted the phase separation mechanism at microscopic level in polymer solution by speeding up the process somewhat. 


\section{CHAPTER 7}

\section{RECOMMENDATIONS}

This chapter provides recommendations for future work, which could be carried out based on this simulation study. This study, for investigation of the effect of thermal diffusion phenomenon or the Ludwig-Soret effect in the phase separation mechanism (TIPS via SD) in polymer solutions under non-uniform temperature field, was carried out using computational technique. The governing mathematical model was solved using numerical methods of Galerkin finite element and finite differences, respectively for space and temporal discretizations, also employing Newton-Raphson iterative mechanism. The computational time taken by each simulation was from $20 \mathrm{hrs}$ to $28 \mathrm{hrs}$ (average). One of the recommendation for future work would be to try to solve the mathematical model using a commercial software such as COMSOL multiphysics, which might help reduce the computational time significantly, and at the same time, smaller mesh sizes could also be employed with it. Another recommendation would be to try and solve the mathematical model using the deeper quenching of the polymer solutions in the unstable region of the phase diagram, and also using the larger temperature gradient across the polymer solution sample for more analysis. 


\section{REFERENCES}

[1] Chan, P. Phase Separation in Polymer Solutions and Blends. Research Signpost, Kerala, India, (2002).

[2] Lee, K.-W. Master's thesis, University of Waterloo, Waterloo, Canada, (2002).

[3] Tran, T. Master's thesis, Ryerson University, Toronto, Canada, (2004).

[4] Chan, P. PhD thesis, McGill University, Montreal, Canada, (1997).

[5] Cowie, J. Polymers: Chemistry and Physics of Modern Materials, $2_{n d}$. Chapman and Hall, New York, (1991).

[6] Chapra, S. and Canale, R. Numerical Methods for Engineers, 5th edition. McGraw Hill, (2005).

[7] Hashimoto, T. Materials Science and Technology, volume 12. VCH, New York, (1993).

[8] Binder, K. Journal of Chemical Physics 79(12), 6387-6409 (1983).

[9] Baker, R. Membrane Technology and Applications. McGraw-Hill, New York, (2000).

[10] Mulder, M. Basic Principles of Membrane Technology, $2^{\text {nd }}$. Kluwer Academic Publisher, Boston, USA, (1996).

[11] Crawford, G., Doane, J., and Zumer, S. Polymer Dispersed Liquid Crystals: Nematic Droplets and Related Systems, chapter 9. in: Handbook of Liquid Crystal Research, Collins, P.J. and Patel, J.S., Eds. Oxford University Press, New York (1997).

[12] Wirtz, D. and Fuller, G. Physical Reveiw Letters 71(14), 2236-2239 (1993). 
[13] Venugopal, G. and Krause, S. Macromolecules 25(18), 4626-4634 (1992).

[14] Wagner, A. and Yeomans, J. Physical Review E 59(4), 4366-4373 (1999).

[15] Lee, K.-W., Kamal, M., and Chan, P. In Annual Technical Conference - ANTEC, Conference Proceedings, volume 1, 342-346, (2007).

[16] Matsuyama, H., Berghmans, S., and Lloyd, D. Polymer 40, 2289-2301 (1999).

[17] Matsuyama, H., Yuasa, M., Kitamura, Y., Teramoto, M., and Lloyd, D. Journal of Membrane Science 179, 91-100 (2000).

[18] Chan, P. Modelling and Simulation in Materials Science and Engineering 14, 41-51 (2006).

[19] Jiang, B. and Chan, P. Macromolecular Theory and Simulations 16, 690-702 (2007).

[20] Caneba, G. and Soong, D. Macromolecules 18(12), 2538-2345 (1985).

[21] Caneba, G. and Soong, D. Macromolecules 18(12), 2545-2555 (1985).

[22] Lee, K.-W., Chan, P., and Feng, X. Chemical Engineering Science 59, 1491-1504 (2004).

[23] Endoh, T. and Tran-Cong, Q. Macromolecular Rapid Communications 20(7), 390-393 (1999).

[24] Tran-Cong, Q., Kawai, J., Nishikawa, Y., and Jinnai, H. Physical Review E 60(2), R1150-R1153 (1999).

[25] Wang, X., Yu, Y.-K., and Taylor, P. Journal of Applied Physics 80(6), 3285-3290 (1996).

[26] Glotzer, S., Di Marzio, E., and Muthukumar, M. Physical Review Letters 74(11), 2034-2039 (1995). 
[27] Drzaic, P. S. Liquid Crystal Dispersions, volume 1 of Series on liquid crystals. World Scientific, (1995).

[28] Chan, P. and Rey, A. Macromolecular Theory and Simulations 4(5), 873-899 (1995).

[29] Higgins, D. Advanced Materials 12(4), 251-264 (2000).

[30] De Groot, S. and Mazur, P. Non-Equilibrium Thermodynamics. Northern Holland Publishing Co. - Amsterdam, (1962).

[31] Kohler, W. and Weigand, S. Thermal Non-Equilibrium Phenomena in Fluid Mixtures, volume LNP584. Springer - Berlin, (2000). Lecture Notes in Physics.

[32] Castro, A. U.S. Patent 4,247,498 (1981).

[33] Chan, P. and Rey, A. Computational Materials Science 3(3), 377-392 (1995).

[34] Tran, T., Chan, P., and Rousseau, D. Chemical Engineering Science 60, 7153-7159 (2005).

[35] Tran, T., Chan, P., and Rousseau, D. Computational Material Science 37, 328-335 (2006).

[36] West, J. Molecular Crystals Liquid Crystals 157(4), 427-441 (1988).

[37] Doane, J., Vaz, N., Wu, B.-G., and Zumer, S. Applied Physics Letters 48(4), 26927 (1986).

[38] Doane, J. Polymer Dispered Liquid Crystal Displays. In: Liquid Crystals: Applications and Uses: Vol. 1:Bahadur, B., ed. World Scientific, New Jersey, USA, (1990). Chapter 14.

[39] Jin, J.-M., Prabhakar, K., and Dao, L. Langmuir 12, 2096-2099 (1996). 
[40] Teixeira, P. and Mulder, B. Journal of Chemical Physics. 105(22), 10145-10152 (1996).

[41] Whitehead, J. and Gill, N. Proceedings of SPIE 5741, 83-89 (2005).

[42] Lloyd, D. Membrane Materials Science - an Overview. in: D.R. Lloyd (Ed.), Material Science of Synthetic Membranes, ACS Symp. Ser. No. 269. American Chemical Society, Washington, DC, (1985).

[43] Kesting, R. Phase Inversion Membranes. in: D.R. Lloyd (Ed.), Material Science of Synthetic Membranes, ACS Symp. Ser. No. 269. American Chemical Society, Washington, DC, (1985).

[44] Strathmann, H. Production of Microporous Media by Phase Inversion Processes. in: D.R. Lloyd (Ed.), Material Science of Synthetic Membranes, ACS Symp. Ser. No. 269. American Chemical Society, Washington, DC, (1985).

[45] Hiatt, W., Vitzthum, G., Wagner, K., Gerlach, K., and Josefiak, C. Microporous Membranes via Critical Temperature Phase Separation. in: D.R. Lloyd (Ed.), Material Science of Synthetic Membranes, ACS Symp. Ser. No. 269. American Chemical Society, Washington, DC, (1985).

[46] Worthy, W. Chemical Engineering News 56, 23 (1978).

[47] Klein, W. Filtration \& Separation 19(2), 130-134 (1982).

[48] Agishi, T. and Mineshima, M. Journal of Membrane Science 44(1), $47-54$ (1989).

[49] Gryta, M. Separation Science and Technology 41(9), 1789-1798 (2006).

[50] Lloyd, D., Kinzer, K., and Tseng, H. Journal of Membrane Science 52(3), 239-261 (1990).

[51] Lloyd, D., Kim, S., and Kinzer, K. Journal of Membrane Science 64(1), 1-11 (1991). 
[52] Kim, S., Lim, B., Alwattari, A., Wang, Y., and Lloyd, D. Journal of Membrane Science 64(1), 41-53 (1991).

[53] Kim, S. and Lloyd, D. Journal of Membrane Science 64(1), 13-29 (1991).

[54] Chiang, C.-Y. and Lloyd, D. Journal of Porous Materials 2, 273-285 (1996).

[55] Graham, P. and McHugh, A. Macromolecules 31, 2565-2568 (1998).

[56] Matsuyama, H., Berghmans, S., Batarseh, M., and Lloyd, D. Journal of Membrane Science 142, 27-42 (1998).

[57] Matsuyama, H., Teramoto, M., Kudari, S., and Kitamura, Y. Journal of Applied Polymer Science 82, 169-177 (2001).

[58] Matsuyama, H., Maki, T., Teramoto, M., and Asano, K. Journal of Membrane Science $204,323-328$ (2002).

[59] Matsuyama, H., Kim, M.-m., and Lloyd, D. Journal of Membrane Science 204, 413419 (2002).

[60] Barton, B., Graham, P., and McHugh, A. Macromolecules 31(5), 1672-1679 (1998).

[61] Barton, B. and McHugh, A. Journal of Membrane Science 166(1), 119-125 (2000).

[62] Graham, P., Pervan, A., and McHugh, A. Macromolecules 30(6), 1651-1655 (1997).

[63] Matsuyama, H., Kudari, S. adn Kiyofuji, H., and Kitamura, Y. Journal of Applied Polymer Science 76(7), 1028-1036 (2000).

[64] Binder, K. Advances in Polymer Science 112, 181 (1994).

[65] Jasnow, D. and Vinlas, J. Physics of Fluids 8(3), 660-669 (1996).

[66] Mao, H., Li, C., Zhang, Y., Bergbreiter, D., and Cremer, P. Journal of American Chemical Society 125(10), 2850-2851 (2003). 
[67] Yamamura, M., Nakamura, S., Kajiwara, T., Kage, H., and Adachi, K. Polymer 44(16), 4689-4704 (2003).

[68] Lee, K.-W., Chan, P., and Feng, X. Macromolecular Theory and Simulations 12(6), 413-424 (2003).

[69] Krekhov, A. and Kramer, L. Physical Review E 70, 061801-061809 (2004).

[70] Voit, A., Krekhov, A., Enge, W., Kramer, L., and Kohler, W. Physical Review Letters 94, 214501-214504 (2005).

[71] Enge, W. and Kohler, W. Chem. Phys. Chem. 5, 395-399 (2004).

[72] Enge, W. and Kohler, W. Physical Chemistry Chemical Physics 6, 2373-2378 (2004).

[73] Rauch, J. and Kohler, W. Journal of Chemical Physics 119(22), 11977-11988 (2003).

[74] Rauch, J. and Kohler, W. Physical Review Letters 88(18), 185901:1-4 (2002).

[75] de Gans, B.-J., Kita, R., and Muller, B. Journal of Chemical Physics 118(17), 8073$8081(2003)$.

[76] Flory, P. Principles of Polymer Chemistry. Cornell University Press, Ithaca, New York, (1953).

[77] Kurata, M. Thermodynamics of Polymer Solutions. Hardwood Academic, New York, (1982).

[78] Cahn, J. Journal of Chemical Physics 42(1), 93-99 (1965).

[79] de Gennes, P.-G. Journal of Chemical Physics 72(9), 4756-4763 (1980).

[80] Jilge, W., Carmesin, I., Kremer, K., and Binder, K. Macromolecules 23(23), 5001$5013(1990)$. 
[81] Elias, H.-G. An Introduction to Polymer Science. VCH, New York, (1997).

[82] Doi, M. and Edwards, S. The Theory of Polymer Dynamics. Oxford University Press, (1986).

[83] Kramer, E., Green, P., and Palmstrom, C. Polymer 25(4), 473-480 (1984).

[84] Elliott, C. and Songmu, Z. Archives for Rational Mechanics and Analysis 96(4), 339-357 (1986).

[85] Novick-Cohen, A. and Segel, L. Physica D 10(3), 277-298 (1984).

[86] Lapidus, L. and Pinder, G. Numerical Solution of Partial Differential Equations in Science and Engineering. A Wiley-Interscience Publication, (1982).

[87] Press, W., Flanner, S., Teukolsky, S., and Vetterling, W. Numerical Recipe: The Art of Scientific Computing. Cambridge Universiy Press, Cambridge, (1992).

[88] Finlayson, B. Nonlinear Analyisi in Chemical Engineering. McGraw Hill Inc., New York, (1980).

[89] Kyu, T., Mustafa, M., Yang, J.-C., and Palffy-Muhoray. Stud. Polym. Sci. 11, 245 (1992).

[90] Tanaka, H., Yokogawa, T., Abe, H., Hayashi, T., and Nishi, T. Physical Review Letters $65,3136(1990)$.

[91] Copetti, M. and Elliott, C. Material Science Technology 6, 273 (1990).

[92] Chakrabarti, A. Physical Review B 45, 9620 (1992).

[93] Brown, G. and Chakrabarti, A. Physical Review E 48, 3705 (1993).

[94] Skripov, V. and Skripov, A. Sov. Phys. Usp. 22(6), 389-410 (1979). 
[95] Lal, J. and Bansil, R. Macromolecules 24, 290 (1991).

[96] Olabisi, O., Robeson, L., and Shaw, M. Polymer-Polymer Miscibility. Academic Press, New York, USA, (1979).

[97] Rauch, J., Hartung, M., Privalov, A., and Kohler, W. Journal of Chemical Physics $126,214901(2007)$. 


\section{APPENDIX A}

\section{DIMENSIONLESS GOVERNING}

\section{EQUATIONS}

The dimensionless governing equation, and initial and boundary conditions are obtained using the following scaling relations:

Dimensionless space:

$$
x^{*}=\frac{x}{L} \quad \text { and } \quad y^{*}=\frac{y}{L}
$$

Dimensionless temperature:

$$
T^{*}=\frac{T}{\theta}
$$

Dimensionless concentration:

$$
c^{*}=c
$$

Dimensionless time:

$$
t^{*}=\frac{2 \nu \kappa t}{\xi L^{4}}
$$

Dimensionless diffusion coefficient:

$$
D^{*}=\frac{k_{B} \theta L^{2}}{2 \nu \kappa}
$$




\section{Dimensionless thermal diffusion coefficient:}

$$
D^{\prime *}=\frac{D^{\prime} \theta \xi L^{2}}{2 \nu \kappa}
$$

where the superscripted asterisks denote dimensionless variables. Consequently, the dimensionless governing equation is expressed as follows:

$$
\begin{aligned}
\frac{\partial c^{*}}{\partial t^{*}} & =D^{*}\left[\left(\frac{3-4 c^{*}+\left(1-2 c^{*}\right) \ln c^{*}}{N_{1}}\right)-\left(\frac{1-4 c^{*}+\left(1-2 c^{*}\right) \ln \left(1-c^{*}\right)}{N_{2}}\right)+4\left(c^{*}-c^{*^{2}}\right)\right. \\
& \left.\cdot(2 \psi-1)-\psi+\frac{1}{2}\right] \nabla^{*} c^{*} \cdot \nabla^{*} T^{*}+D^{*} T^{*}\left[\frac{-1}{N_{1}}+\frac{1}{N_{2}}-2 \chi\left(1-2 c^{*}\right)\right]\left(\nabla^{*} c^{*}\right)^{2} \\
& -\left(1-2 c^{*}\right) \nabla^{*} c^{*} \cdot \nabla^{*^{3}} c^{*}+D^{*} T^{*}\left[\frac{\left(1-c^{*}\right)}{N_{1}}+\frac{c^{*}}{N_{2}}-2 \chi\left(c^{*}-c^{*^{2}}\right)\right] \nabla^{*^{2}} c^{*} \\
& -\left(c^{*}-c^{*^{2}}\right) \nabla^{*^{4}} c^{*}+D^{\prime *}\left(1-2 c^{*}\right) \nabla^{*} c^{*} \cdot \nabla^{*} T^{*}
\end{aligned}
$$

In addition, the dimensionless initial and boundary conditions are:

$$
\begin{gathered}
c^{*}\left(x^{*}, y^{*}, t^{*}=0\right)=c_{0}^{*}+\delta c^{*}\left(x^{*}, y^{*}, t^{*}=0\right) \\
\frac{\partial c^{*}}{\partial x^{*}}=0 \quad \text { at } t^{*}>0, \text { and } x^{*}=0 \text { and } x^{*}=1 \\
\frac{\partial c^{*}}{\partial y^{*}}=0 \quad \text { at } t^{*}>0, \text { and } y^{*}=0 \text { and } y^{*}=1 \\
\frac{\partial^{3} c^{*}}{\partial x^{* 3}}+\frac{\partial^{3} c^{*}}{\partial x^{*} \partial y^{*^{2}}}=0 \quad \text { at } t^{*}>0, \text { and } x^{*}=0 \text { and } x^{*}=1 \\
\frac{\partial^{3} c^{*}}{\partial y^{* 3}}+\frac{\partial^{3} c^{*}}{\partial x^{* 2} \partial y^{*}}=0 \quad \text { at } t^{*}>0, \text { and } y^{*}=0 \text { and } y^{*}=1
\end{gathered}
$$




\section{APPENDIX B}

\section{ESTIMATION OF ORDER OF}

\section{MAGNITUDE}

Order of magnitude estimate for the dimensionless diffusion coefficient, $D^{*}$ and dimensionless thermal diffusion coefficient, $D^{\prime *}$ can be obtained using the typical parameter values listed in Table B.1 for polymer systems (i.e. polystyrene-polyvinylmethylether blend, polystyrene-cyclohexane and polystyrene-toluene solutions) in the early and the intermediate stages of the spinodal decomposition and the following expressions:

$$
\begin{aligned}
D^{*} & =\frac{k_{B} \theta L^{2}}{2 \nu \kappa} \\
D^{\prime *} & =\frac{D^{\prime} \theta \xi L^{2}}{2 \nu \kappa}
\end{aligned}
$$

where $k_{B}$ is Boltzmann's constant, $\kappa$ is a positive constant related to interfacial constant, $\nu$ is the volume of a cell or a segment, $\theta$ is the theta temperature, $\xi$ is the frictional coefficient, $D^{\prime}$ is the thermal diffusion coefficient, and $L$ is the length of the geometry, which is $5 \times 10^{-6}$ in this study. 
Table B.1: Parameter values

\begin{tabular}{lrc}
\hline Parameter & Value & Reference \\
\hline$N_{1}$ & 1 & This Study \\
$N_{2}$ & 10 & This Study \\
$\psi$ & 1 & {$[4]$} \\
$T(\mathrm{~K})$ & 298 & This Study \\
$\theta(\mathrm{K})$ & 300 & {$[76,5]$} \\
$\chi$ & 1.1667 & {$[4]$} \\
$c_{c}^{*}$ & 0.7597 & {$[4]$} \\
$T_{c}^{*}$ & 0.7319 & {$[4]$} \\
$\chi_{c}^{*}$ & 0.8662 & {$[4]$} \\
$L\left(\mathrm{~m} \mathrm{~N}^{*} 0^{-6}\right)$ & 5 & This Study \\
$\nu\left(\mathrm{m}^{3} \mathrm{segment}^{-1} \times 10^{-27}\right)$ & 1 & {$[95]$} \\
$k_{B}\left(\mathrm{~J} \mathrm{~K}^{-1} \times 10^{-23}\right)$ & 1.21 & {$[96]$} \\
$\kappa\left(\mathrm{J} \mathrm{m}^{-1} \times 10^{-7}\right)$ & 0.92 & {$[97]$} \\
$D\left(\mathrm{~m}^{2} \mathrm{~s}^{-1} \times 10^{-10}\right)$ & 1.3806504 & Universal Constant \\
$D^{\prime}\left(\mathrm{m}^{2} \mathrm{~s}^{-1} \mathrm{~K}^{-1} \times 10^{-11}\right)$ & 1 & {$[97]$} \\
\hline
\end{tabular}

